\title{
Metanálise caso a caso sob a perspectiva bayesiana
}

\author{
Camila Bertini Martins
}

TESE APRESENTADA

$\mathrm{AO}$

Instituto De Matemática e Estatística

DA

UNIVERSIDADE DE SÃO PAUlO

PARA

OBTENÇÃO DO TÍTULO

$\mathrm{DE}$

Doutor EM CiÊnCIAS

\section{Programa: Estatística \\ Orientador: Prof. Dr. Carlos Alberto de Bragança Pereira}

Durante o desenvolvimento deste trabalho, o autor recebeu auxílio financeiro da CAPES/CNPq

São Paulo, novembro de 2013 


\section{Metanálise caso a caso sob a perspectiva bayesiana}

Esta é a versão original da tese elaborada pela candidata Camila Bertini Martins, tal como submetida à Comissão Julgadora. 


\title{
Metanálise caso a caso sob a perspectiva Bayesiana
}

\author{
Esta tese contém as correções e alterações \\ sugeridas pela Comissão Julgadora durante a defesa \\ realizada por Camila Bertini Martins em 29/11/2013. \\ O original encontra-se disponível no Instituto de \\ Matemática e Estatística da Universidade de São Paulo.
}

Comissão Julgadora:

- Prof. Dr. Carlos Alberto de Bragança Pereira (orientador) - IME-USP

- Profa. Dra. Viviana Giampaoli - IME-USP

- Prof. Dr. Adriano Polpo de Campos - UfSCar

- Prof. Dr. Hélio Elkis - FM-USP

- Prof. Dr. Eduardo Massad - FM-USP 


\section{Agradecimentos}

Primeiramente agradeço à Deus, pela vida e por me dar forças durante esses anos de estudos.

Aos meus pais e irmã, Sonia, Antônio e Ana Carolina, os mais profundos agradecimentos por suas sábias lições de vida, esperança, compreensão e acima de tudo pelo amor.

À minha avó de saudosa memória, Irene, que sempre apoiou-me.

Ao meu marido, Mateus, pelo apoio, carinho, dedicação e grande paciência.

Ao meu orientador, Prof. Dr. Carlos Alberto de Bragança Pereira, por acreditar em mim, apoiando-me e incentivando-me sempre, pela sua compreensão, dedicação e amizade. O Prof. Carlinhos não contribuiu apenas em minha tese mas sim, na minha formação profissional e pessoal.

Ao Prof. Dr. Adriano Polpo de Campos, pela imprescindível contribuição em minha tese, pelo apoio, compreensão, paciência e, principalmente, pela amizade.

Aos membros da Comissão Julgadora pelas valiosas sugestões e comentários dados na realização do exame de qualificação e na defesa.

Ao meu orientador de programa, Prof. Dr. Luis Gustavo Esteves, pelo apoio, compreensão e amizade no momento que mais precisei.

Aos pesquisadores Dr. Hélio Elkis e Miroslav Herceg, por fornecerem os dados reais de suas pesquisas, muito importantes nesta tese.

À minha tia e madrinha, Lourdes, pelo apoio em todos os momentos de minha vida.

Ao melhor amigo que alguém poderia ter, André Yoshizumi, pelo apoio e companheirismo nos momentos mais difíceis da minha vida.

Ao amigo e Prof. Victor Fossaluza pela contribuição com este trabalho e pela companhia em grande parte da minha jornada ao longo desses anos de doutorado.

Ao amigo e Prof. Eduardo Nakano que inúmeras vezes me auxiliou nas mais diversas situações durante meu doutorado.

Aos meus amigos Rafael Izbicki e Tiago Mendonça pela grande troca de experiência, companheirismo e por fazerem meus dias mais alegres.

Aos amigos, Tiago Vargas, Joelmir Borssoi, Wagner Barreto, Daniel Mendes, Silvio Rodrigues, Rafael Farias e Fran Lima que me acompaharam nesta caminhada, compartilhando momentos de sofrimentos e alegrias durante todos esses anos de doutorado.

A todos os meus amigos pelo companheirismo, pela ajuda nas horas difíceis e pelos momentos de descontração.

A todos os professores e funcionários do Instituto de Matemática e Estatística da USP que contribuíram para minha formação.

À CAPES e ao CNPq, pelo apoio financeiro. 


\section{Resumo}

O papel da metanálise de sumarizar estudos publicados de mesmo objetivo, por meio da estatística, torna-se cada dia mais fundamental em razão do avanço da ciência e do desejo de usar o menor número de seres humanos em ensaios clínicos, desnecessários, em vários casos. A síntese das informações disponíveis facilita o entendimento e possibilita conclusões robustas. O aumento de estudos clínicos, por exemplo, promove um crescimento da necessidade de metanálises, fazendo com que seja necessário o desenvolvimento de técnicas sofisticadas. Desse modo, o objetivo deste trabalho foi propor uma metodologia bayesiana para a realização de metanálises. O procedimento proposto consiste na mistura das distribuições a posteriori do parâmetro de interesse de cada estudo pertencente à metanálise; ou seja, a medida metanalítica proposta foi uma distribuição de probabilidade e não uma simples medida-resumo. A metodologia apresentada pode ser utilizada com qualquer distribuição a priori e qualquer função de verossimilhança. O cálculo da medida metanalítica pode ser utilizado, desde problemas simples até os mais sofisticados. Neste trabalho, foram apresentados exemplos envolvendo diferentes distribuições de probabilidade e dados de sobrevivência. Em casos, em que se há uma estatística suficiente disponível para o parâmetro em questão, a distribuição de probabilidade a posteriori depende dos dados apenas por meio dessa estatística e, assim, em muitos casos, há a redução de dimensão sem perda de informação. Para alguns cálculos, utilizou-se o método de simulação de Metropolis-Hastings. O software estatístico utilizado neste trabalho foi o $R$.

Palavras-chave: Análise de sobrevivência, Dados censurados, Inferência bayesiana, Metanálise, Modelo Weibull. 


\section{Abstract}

The meta-analysis role of using Statistics to summarize published studies that have the same goal becomes more essential day by day, due to the improvement of Science and the desire of using the least possible number of human beings in clinical trials, which in many cases is unnecessary. By match the available information it makes the understanding easier and it leads to more robust conclusions. For instance, the increase in the number of clinical researches also makes the need for meta-analysis go higher, arising the need for developing sophisticated techniques. Then our goal in this work is to propose a Bayesian methodology to conduct meta-analysis. The proposed procedure is a blend of posterior distributions from interest parameters of each work we are considering when doing meta-analysis. As a consequence, we have a probability distribution as a meta-analytic measure, rather than just a statistical summary. The methodology we are presenting can be used with any prior probability distribution and any likelihood function. The calculation of the metaanalytic measure has its uses from small to more complex problems. In this work we present some examples that consider various probability distributions and also survival data. There is a sufficient statistic available for the parameter of interest, the posterior probability distribution depends on the data only through this statistic and thus, in many cases, we can reduce our data without loss of information. Some calculations were performed through Metropolis-Hastings simulation algorithm. The statistical software used in this work was the R.

Keywords: Survival analysis, Censored data, Bayesian inference, Meta-analysis, Weibull model. 


\section{Sumário}

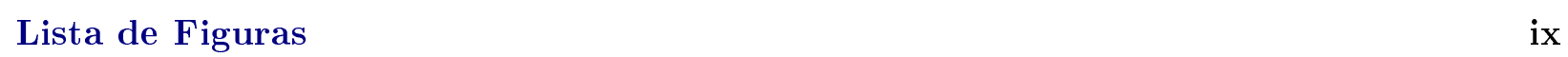

Lista de Tabelas $\quad$ xiii

1 Introdução $\quad \mathbf{1}$

1.1 Organização do trabalho . . . . . . . . . . . . . . . . . . 4

2 Distribuição metanalítica $\quad \mathbf{5}$

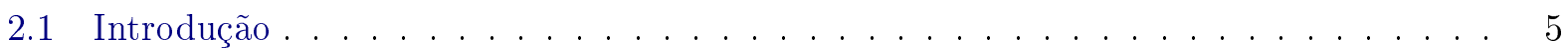

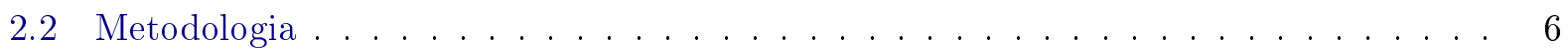

2.2 .1 Generalização do método proposto . . . . . . . . . . . . . 11

2.3 Aplicação . . . . . . . . . . . . . . . . . . . . . . . 17

2.3.1 Exemplo 3: Metanálise para proporção . . . . . . . . . . . . . . 17

2.3.2 Exemplo 4: Metanálise para diferença entre médias . . . . . . . . . . . . 21

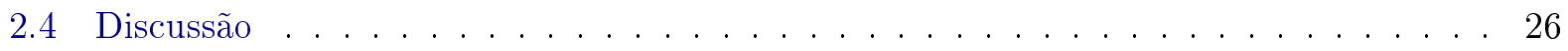

3 Metanálise para dados de sobrevivência $\quad 27$

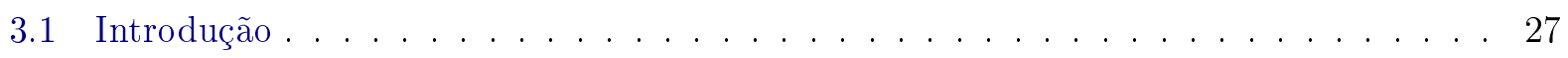

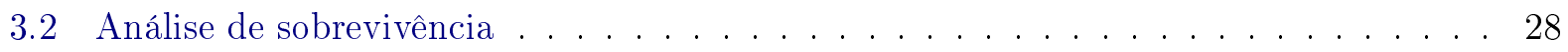

3.2.1 Construção da função de verossimilhança para dados censurados . . . . . . . 29

3.3 Metanálise para dados de sobrevivência . . . . . . . . . . . . . . 30

3.4 Aplicação . . . . . . . . . . . . . . . . . . . 31

3.4.1 Exemplo 1: Modelo Exponencial . . . . . . . . . . . . . . . . 31

3.4 .2 Exemplo 2: Modelo Log-normal . . . . . . . . . . . . . . . . . . . 36

3.4 .3 Exemplo 3: Modelo Weibull . . . . . . . . . . . . . . . . . . . . 42

3.4.4 Exemplo 4: Metanálise com modelos de riscos competitivos . . . . . . . . 47

3.4.5 Exemplo 5: Modelos de regressão em análise de sobrevivência . . . . . . . . . 55

3.5 Discussão . . . . . . . . . . . . . . . . . . . . . . 60

$\begin{array}{lll}4 & \text { Considerações finais e pesquisas futuras } & 61\end{array}$

$\begin{array}{lll}\text { A Anexo } & 63\end{array}$

$\begin{array}{ll}\text { Referências Bibliográficas } & 67\end{array}$ 
viii SUMÁRIO 


\section{Lista de Figuras}

2.1 (a) Curvas de nível da distribuição conjunta a posteriori de $\left(\mu, \sigma^{2}\right)$ em cada estudo.

(b) Curvas de nível da distribuição a posteriori metanalítica de $\left(\mu, \sigma^{2}\right)$. (c) Gráfico tridimensional da distribuição a posteriori metanalítica de $\left(\mu, \sigma^{2}\right)$. (d) Região HPD de $95 \%$ de credibilidade para $\left(\mu, \sigma^{2}\right) . \ldots \ldots \ldots \ldots$

2.2 Forest Plot para os dados do Exemplo 1. . . . . . . . . . . . . . . . . 9

2.3 (a) Distribuições a posteriori marginal de $\mu$. (b) Resultados da metanálise proposta (distribuição a posteriori metanalítica padronizada) e da metanálise usual (diamante). 10

2.4 (a) Curvas de nível da distribuição a posteriori conjunta de $\mu$ e $\sigma^{2}$. (b) Distruibuição a posteriori marginal de $\mu \ldots \ldots \ldots \ldots \ldots \ldots \ldots \ldots$

2.5 Forest Plot para a proporção de sucessos no uso da droga SAMe. . . . . . . . . . . 13

2.6 Análise combinada para a proporção de sucessos. (Os nove pontos correspondem às proporções observadas para os nove estudos, com as áreas dos pontos proporcionais aos tamanhos amostrais.) . . . . . . . . . . . . . . . . . . 14

2.7 Densidade a posteriori estimada da proporção dos sucessos, considerando priori Uniforme (2.18).Como na Figura 2.6, os pontos correspondem às proporções observadas. 15

2.8 (a) Distribuições a posteriori de $p$ para os nove estudos. (b) Distribuição a posteriori metanalítica de $\ldots_{1} \ldots \ldots \ldots \ldots \ldots \ldots \ldots \ldots \ldots$

2.9 Distribuições a posteriori de $p \ldots \ldots \ldots \ldots \ldots \ldots \ldots \ldots \ldots$

2.10 (a) Distribuições a posteriori de $p_{1}$. (b) Distribuições a posteriori de $p_{2} . \quad \ldots \ldots$. . 20

2.11 Distribuições a posteriori metanalíticas. . . . . . . . . . . . . . . . . . 20

2.12 Curvas de nível. . . . . . . . . . . . . . . . . . . . . . . . . 22

2.13 (a) Região HPD de 95\% de credibilidade para o grupo experimental. (b) Região HPD de $95 \%$ de credibilidade para o grupo controle. (c) Região HPD de $95 \%$ de credibilidade apenas para os parâmetros metanalíticos. (d) Curvas de nível das distribuições a posteriori metanalíticas de ambos os grupos. . . . . . . . . . . . . . . . 23

2.14 Distribuição a posteriori metanalítica de $\mu$ e $\sigma^{2}$ para o grupo experimental (a) e para o grupo controle $(\mathrm{b}) . \ldots \ldots \ldots \ldots \ldots \ldots$

2.15 (a) Curvas de nível das distribuições a posteriori de $(\delta, \tau)$ para cada estudo. (b) Curvas de nível da distribuição a posteriori metanalítica de $(\delta, \tau)$. (c) Distribuição a posteriori metanalítica de $(\delta, \tau)$ em 3D. (d) Região HPD de $95 \%$ de credibilidade. . . 25

2.16 Distribuições a posteriori marginal de $\delta \ldots \ldots \ldots \ldots \ldots \ldots \ldots$

3.1 (a) Distribuição do tempo de vida do estudo 1. (b) Distribuição do tempo de vida

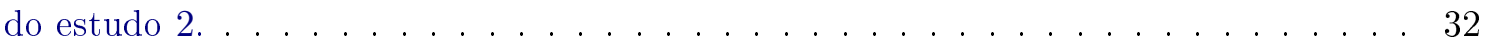


3.2 Curvas de sobrevivência para o Estudo 1 (a) e para o Estudo 2 (b) . . . . . . . . . . . 33

3.3 (a) Distribuições a posteriori de $\theta$. (b) Curva de sobrevivência metanalítica. . . . . . . 34

3.4 Curvas de sobrevivência relacionadas aos estudos 1 (a) e 2 (b), respectivamente. . . . 35

3.5 (a) Distribuições a posteriori de $\theta$. (b) Curvas de sobrevivência. . . . . . . . . . . . 36

3.6 (a) Função densidade Log-normal, (b) Função de sobrevivência Log-normal e (c) Função de risco Log-normal. . . . . . . . . . . . . . . . . . . . . . . . 37

3.7 (a) Distribuição dos tempos de vida do Estudo 1. (b) Distribuição dos tempos de vida do Estudo 2. (c) Distribuição dos tempos de vida do Estudo 3. . . . . . . . . . . 38

3.8 Curvas de sobrevivência para o Estudo 1 (a), para o Estudo 2 (b) e para o Estudo 3

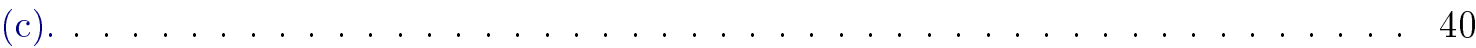

3.9 (a) Curvas de nível das distribuições a posteriori de $(\mu, \sigma)$ para cada estudo. (b) Curvas de nível da distribuição a posteriori metanalítica de $(\mu, \sigma)$. (c) Distribuição a posteriori metanalítica de $(\mu, \sigma)$ em 3D. (d) Região HPD de $95 \%$ de credibilidade. . 41

3.10 (a) Curva de sobrevivência metanalítica. (b) Curvas de sobrevivência para o problema.

3.11 (a) Função densidade do modelo Weibull $(\eta=1)$. (b) Função de confiabilidade do modelo Weibull $(\eta=1)$. (c) Função de risco do modelo Weibull $(\eta=1)$. . . . . . . 43

3.12 (a) Distribuição dos tempos de vida do Estudo 1. (b) Distribuição dos tempos de vida do Estudo 2. . . . . . . . . . . . . . . . . . . . . . . . 44

3.13 Curvas de sobrevivência para o Estudo 1 (a) e para o Estudo 2 (b) . . . . . . . . . . . 45

3.14 (a) Densidades a posteriori estimadas de $\beta$. (b) Densidades a posteriori estimadas de

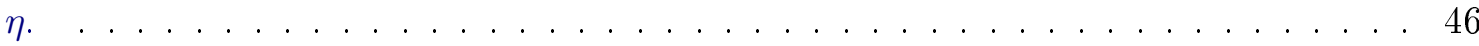

3.15 Curvas de sobrevivência para o problema. . . . . . . . . . . . . . . 46

3.16 Funções de risco para o problema. . . . . . . . . . . . . . . . 46

3.17 Distribuições a posteriori estimadas dos parâmetros do fator de risco 1 ((a) e (b)), do fator de risco 2 ((c) e (d)) e do fator de risco 3 ((e) e (f)). . . . . . . . . . 50

3.18 Ajustes referentes ao Estudo 1. (a) Ajuste da função de sobrevivência dos fatores de

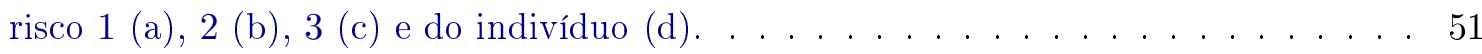

3.19 Ajustes referentes ao Estudo 2. (a) Ajuste da função de sobrevivência dos fatores de

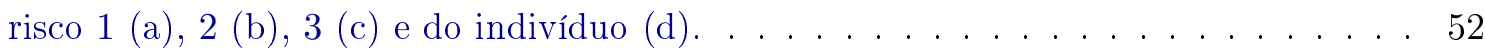

3.20 Ajustes referentes ao Estudo 3. (a) Ajuste da função de sobrevivência dos fatores de risco 1 (a), 2 (b), 3 (c) e do indivíduo $(\mathrm{d}) \ldots \ldots \ldots \ldots \ldots \ldots$

3.21 (a) Curva de sobrevivência metanalítica para o fator de risco 1 e banda de credibilidade de 95\%. (b) Curvas de sobrevivência relacionadas ao Fator 1. . . . . . . . . . . 53

3.22 (a) Curva de sobrevivência metanalítica para o fator de risco 2 e banda de credibilidade de 95\%. (b) Curvas de sobrevivência relacionadas ao Fator 2. . . . . . . . . . 54

3.23 (a) Curva de sobrevivência metanalítica para o fator de risco 3 e banda de credibilidade de 95\%. (b) Curvas de sobrevivência relacionadas ao Fator 3. . . . . . . . . . . 54

3.24 (a) Curva de sobrevivência metanalítica do indivíduo e banda de credibilidade de 95\%. (b) Curvas de sobrevivência do indivíduo. . . . . . . . . . . . . 55

3.25 Distribuição do tempo de re-hospitalização (anos) em ambos os estudos. . . . . . . . 56

3.26 (a) Distribuições a posteriori de $\beta$. (b) Distribuições a posteriori de $\alpha_{0}$. (c) Distribuições a posteriori de $\alpha_{1} \ldots \ldots \ldots \ldots \ldots \ldots \ldots \ldots$ 
3.27 Curvas de sobrevivência relacionadas aos Estudos 1 (a), Estudo 2 (b) e metanálise (c). 60 


\section{Lista de Tabelas}

1.1 Exemplo de análise combinada - revisão da Cochrane. . . . . . . . . . . . . . . 3

2.1 Sucessos observados no uso da droga antidepressiva SAMe. . . . . . . . . . . . . . . 12

2.2 Ajuste do modelo de mistura finita para os dados da Tabela $2.1 \ldots$. . . . . . . . 16

2.3 Prevalência do sucesso da nova técnica cirúrgica com relação à usual. . . . . . . . . . 18

2.4 Sumário a posteriori de $p$ para cada centro. . . . . . . . . . . . . . . . . 19

2.5 Sumário da distribuição a posteriori metanalítica de $p \ldots \ldots \ldots \ldots$. . . . . . . 19

2.6 Medidas resumo da PAS. . . . . . . . . . . . . . . . . . . . . . . 21

3.1 Medidas-resumo do tempo de vida de ambos os estudos. . . . . . . . . . . . . . . 32

3.2 Medidas-resumo a posteriori de $\theta$ para ambos os estudos. . . . . . . . . . . . 33

3.3 Medidas-resumo a posteriori de $\theta$ para ambos os estudos com dados censurados. . . . 35

3.4 Medidas-resumo do tempo de sobrevivência em cada estudo pertencente à metanálise. 37

3.5 Medidas-resumo do tempo de sobrevivência para ambos os estudos pertencentes à

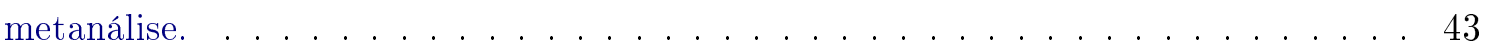

3.6 Medidas-resumo a posteriori para os parâmetros do modelo Weibull. . . . . . . . . . 45

3.7 Medidas-resumo a posteriori para os parâmetros metanalíticos. . . . . . . . . . . 45

3.8 Parâmetros do modelo Weibull. . . . . . . . . . . . . . . . . . . 48

3.9 Medidas-resumo do tempo de sobrevivência independente do fator de risco em cada estudo pertencente à metanálise. . . . . . . . . . . . . . . . . . 48

3.10 Medidas-resumo a posteriori para os parâmetros relacionados ao fator de risco 1. . 49

3.11 Medidas-resumo a posteriori para os parâmetros relacionados ao fator de risco 2 . . 49

3.12 Medidas-resumo a posteriori para os parâmetros relacionados ao fator de risco 3. . 49

3.13 Calculo da probabilidade de um indivíduo sobreviver mais que o tempo 5 . . . . . . 55

3.14 Medidas-resumo das variáveis Tempo de re-hospitalização (anos) e Idade. . . . . . . . 56

3.15 Frequência e percentual das variáveis Gênero, Tipo de antipsicótico e Censura. . . 56

3.16 Medidas-resumo a posteriori para os parâmetros de interesse. . . . . . . . . . 58

A.1 Dados referente ao Exemplo 2 - Capítulo $3 \ldots \ldots \ldots$

A.2 Dados referente ao Exemplo 3 - Capítulo $3 \ldots \ldots \ldots$. . . . . . . . . 63

A.3 Dados referente ao Exemplo 1 - Capítulo $3 \ldots \ldots \ldots$. . . . . . . . . . 64

A.4 Dados referente ao Exemplo 4 - Capítulo $3 \ldots \ldots \ldots$. . . . . . . . . 65 


\section{Capítulo 1}

\section{Introdução}

O crescimento na produção e na disponibilização das informações científicas e a necessidade de aproveitar a massa de informações disponíveis produzem novas tecnologias de sistematização. Dessas informações, todas as que forem úteis devem ser utilizadas para se obter evidências mais fidedignas. Assim, um modo de combinar esses resultados é por meio de uma revisão sistemática.

Uma revisão sistemática é um tipo de pesquisa planejada para sumarizar, em geral, estudos médicos, respondendo uma questão clínica específica. Utilizam-se métodos apropriados para identificar, selecionar e avaliar criticamente os estudos e também para analisar os dados desses estudos incluídos na revisão. Em uma revisão sistemática, os dados dos diferentes estudos podem ser quantitativamente combinados por métodos estatísticos, que são chamados de técnicas de metanálise (Martinez [2007]). Com a grande quantidade de publicações, há necessidade de sintetizá-las para facilitar o acesso aos conjuntos de informações com o objetivo de conseguir conclusões mais robustas. Consequentemente, o papel da metanálise de sumarizar estudos publicados, por meio da estatística, torna-se cada dia mais fundamental.

O termo metanálise aparece com muitos problemas de definição na literatura. Às vezes, significa uma revisão completa, incluída a parte da revisão sistemática e da combinação dos resultados e, outras vezes, o termo é restringido à revisão sistemática. Há, também, autores que descrevem metanálise, como uma revisão sistemática quantitativa (Lau et al. [1997]), e outros, mais especificamente, como a combinação estatística de, pelo menos, dois estudos, para produzir uma estimativa única. Eventualmente, é possível encontrar revisões que não apresentem sínteses quantitativas, sendo intituladas de metanálise (Campbell et al. [2004]). Até onde se sabe o termo metanálise foi utilizado pela primeira vez por Glass [1976], que o define como uma análise estatística de uma coleção de resultados provenientes de estudos individuais, com o propósito de complementar, o que já havia sido descrito. No entanto, a combinação estatística dos dados provenientes de diversos estudos de mesmo objetivo já era utilizada, antes mesmo de se definir o termo metanálise. A primeira, é atribuída a Pearson, em 1904, na qual foram analisados dados provenientes de cinco estudos na verificação da correlação entre a vacina contra a febre entérica e sua mortalidade (Leandro [2005]). Na década de 30, Tippett [1931], Fisher [1932], Cochran [1937] e Pearson [1938] descreveram técnicas estatísticas para a combinação de dados provenientes de diferentes estudos. Durante os anos de 1970 e 1980, cientistas sociais, incluindo Rosenthal [1978], Glass [1981], Hedges [1982], Hedges [1983], Hunter et al. [1982], Light [1983] e Light e Pillemar [1984], popularizaram a metanálise e, posteriormente, desenvolveram métodos estatísticos para sua aplicação. Estes pesquisadores expandiram o objetivo dos estudos que combinam dados para incluir a tentativa de identificar sistematicamente os estudos a serem combinados e também desenvolveram a estimação do tamanho do efeito (medida de força de um fenômeno/ estatística descritiva), um objetivo fundamental da metanálise baseada na literatura (Petitti [1994]).

DerSimonian e Laird [1986] definem metanálise, como uma análise estatística de uma coleção de resultados analíticos com o propósito de integrar seus achados. Ao se comparar grupos caso/ controle, por exemplo, os estudos, muitas vezes, chegam com restrições de tamanho de amostra ou tipo de seleção. Ao combinar resultados de muitos desses estudos, objetiva-se aumentar o poder 
das conclusões e contornar muitas dessas restrições. O uso de técnicas estatísticas para combinar os resultados de estudos similares pode melhorar a precisão das estimativas do efeito de tratamento, por exemplo, e pode servir para avaliar se estes são ou não similares. A decisão de se considerar os vários estudos como similares na concepção é, na verdade, essencial para a validade dos resultados combinados.

A metanálise oferece a oportunidade de conciliar diferenças entre regiões, países e grupos e determinar um efeito médio estimado sobre os estudos. Essa conciliação vem sendo com frequência utilizada em ensaios clínicos para estimar o efeito médio de tratamentos clínicos em vários grupos. Métodos correntes em metanálise permitem uma investigação dos efeitos em estudos diferentes para determinar um impacto global de um futuro tratamento (Dutton [2011]). Se bem conduzida, uma metanálise permite uma avaliação mais objetiva sobre a evidência de utilidade de um tratamento, podendo antecipar a introdução de um tratamento eficiente na prática clínica (Egger e Smith [1997]). De acordo com Atallah [1998], uma revisão sistemática não leva menos de 3 meses, quando conduzida por profissionais experientes, podendo ultrapassar o período de 1 ano, sendo praticamente impossível ser realizada por apenas um pesquisador.

Para que se tenha uma metanálise de qualidade é importante que os estudos a serem combinados tenham sido avaliados com cuidado, para que haja confiança em sua associação. Para isso, não são suficientes apenas métodos estatísticos, pois estes não podem avaliar metodologicamente cada estudo incluído na metanálise. Portanto, é preciso que critérios de seleção de estudos sejam estabelecidos. Estes critérios devem ser bem definidos e rigorosos para não aumentar indevidamente a variabilidade das variáveis importantes (Justo et al. [2005]).

O modo usual de se fazer metanálise é considerar apenas os resultados de cada estudo, e então, combiná-los por meio de algum método. Este tipo de metanálise, chamado de metanálise baseada na literatura, leva em consideração apenas os resultados obtidos nos estudos individuais. Ou seja, extrai de cada estudo selecionado uma medida de efeito para então, combiná-las e encontrar uma única medida metanalítica. Esta medida nada mais é do que uma média ponderada das medidas de efeitos de cada estudo (Mantel e Haenszel [1959]; DerSimonian e Laird [1986]).

É importante ressaltar que metanálise não é o mesmo que análise combinada de dados, ou seja, considerar os indivíduos de todos os estudos, como se fossem parte de um grande estudo é perder a variabilidade que, possivelmente, exista entre esses estudos. Uma revisão da Cochrane sobre estudos de creche para crianças pré-escolares incluiu dois experimentos (Alderson e Green [2002]). Neste exemplo (Tabela 1.1), o foco está no resultado se uma criança foi retida na mesma série, após um período no grupo tratamento de creche ou no grupo controle de não creche. No primeiro experimento, a diferença entre riscos é $-0,16$, evidenciando que o tratamento creche parece promissor. No segundo experimento, o risco absoluto de ser retido na mesma série é consideravelmente menor; no entanto, a diferença entre riscos embora pequena, ainda evidencia um benefício da creche. Entretanto, ao ignorar a informação de que as crianças são de experimentos diferentes e ao considerar que todas pertencem a uma única amostra, o resultado seria diferente, evidenciando que a creche seria prejudicial às crianças. Este é o chamado paradoxo de Simpson e, por isso, que não se deve juntar diretamente os participantes de cada estudo. A primeira regra da metanálise é manter os indivíduos dentro de cada estudo, preservando os efeitos da aleatorização para compará-los de igual para igual. Portanto, deve-se fazer a comparação dentro de cada experimento e, então, combinar as conclusões. Isto é, calcula-se, para cada estudo, uma medida de efeito de tratamento antes de realizar uma metanálise para, então, encontrar a estimativa do efeito combinado sobre os estudos. 
Tabela 1.1: Exemplo de análise combinada - revisão da Cochrane.

\begin{tabular}{ccccccc}
\hline Estudo & \multicolumn{2}{c}{ Experimento 1 } & \multicolumn{2}{c}{ Experimento 2 } & \multicolumn{2}{c}{ Análise Combinada } \\
\hline \hline & Creche & Não creche & Creche & Não creche & Creche & Não creche \\
Retido & 19 & 13 & 6 & 7 & 25 & 20 \\
Total & 36 & 19 & 58 & 65 & 94 & 94 \\
Risco & 0,528 & 0,684 & 0,1034 & 0,1077 & 0,266 & 0,238 \\
Diferença entre Riscos & \multicolumn{2}{c}{$-0,16$} & \multicolumn{2}{c}{$-0,004$} & \multicolumn{2}{c}{$+0,03$} \\
\hline
\end{tabular}

Como referido anteriormente, o modo usual de se fazer metanálise é considerar apenas as medidas de efeito de cada estudo e, então, combiná-las utilizando a média ponderada desses efeitos. Contudo, existem algumas limitações no uso da metanálise baseada na literatura: estudos publicados podem utilizar métodos diferentes, criando dificuldades na combinação dos resultados; diferenças no planejamento do estudo podem dificultar a justificativa do uso da técnica (Dutton [2011]); revisões que envolvem toda a literatura relevante sobre um determinado assunto, podem acarretar um viés natural de publicação que limita o número de estudos a serem incluídos na análise; estudos pequenos e que não mostram significância estatística podem não estar representados na literatura publicada e se apenas medidas-resumo são apresentadas na literatura, pode ser impossível a realização de certos tipos de análises, como por exemplo, a metanálise com dados de sobrevivência (Stewart e Tierney [2002]). Como uma alternativa à este tipo de metanálise, tem-se a metanálise caso a caso, que trabalha com os dados individuais de cada caso/paciente.

Stewart e Parmar [1993] comparam os dois métodos, utilizando dados do grupo de câncer de ovário avançado que mostram resultados da comparação de dois tipos de tratamento para o câncer (drogas não usuais e quimioterapia). Para a metanálise baseada na literatura, as razões de riscos foram estimadas por meio das publicações das curvas de sobrevivência do $30^{\circ}$ mês e a medida metanalítica, pelo método de Mantel-Haenszel. Para a metanálise caso a caso, as razões de risco foram calculadas diretamente dos dados brutos de cada estudo. Considerando que a metanálise caso a caso inclui todos os indivíduos, os resultados mostraram melhora de 2,5\% nas taxas de sobrevivência no $30^{\circ}$ mês, enquanto a metanálise baseada na literatura mostrou melhora de $7,5 \%$. A metanálise baseada na literatura forneceu uma estimativa do efeito do tratamento três vezes maior que a metanálise caso a caso. Viés de publicação, exclusão de pacientes, tempo de acompanhamento e o método da análise contribuíram para esta diferença observada. Notou-se que a metanálise caso a caso reduziu o viés de publicação das análises baseadas na literatura.

A metanálise caso a caso, ao invés de trabalhar com os resultados dos estudos publicados, os dados originais de cada participante de um estudo considerado são solicitados diretamente aos pesquisadores responsáveis pelo estudo em questão. Estes dados poderão ser reanalisados e, se apropriados, combinados na metanálise (Higgins e Greens [2008]). Esta abordagem requer habilidades particulares e, geralmente, leva mais tempo que a metanálise convencional. Entretanto, este tipo de metanálise oferece benefícios relacionados com a qualidade dos dados e com os tipos de análises que podem ser realizadas e, por esta razão, é considerada o padrão ouro de metanálise (Dutton [2011]). Esta técnica por envolver a coleção dos dados brutos de cada estudo de interesse pode realizar análises de subgrupos, combinar diferentes escalas de medidas e ainda permite a verificação dos resultados por meio da exploração e da reanálise dos dados de um modo consistente. Portanto, o pesquisador ao ter acesso aos dados completos de cada estudo pode solucionar alguns dos problemas tradicionais da metanálise baseada na literatura. A metanálise caso a caso deveria ser utilizada em todas as situações, pois possibilita todos os tipos de análise dos dados fornecendo resultados mais precisos. Entretanto, existem circunstâncias em que não se pode ter acesso aos dados dos estudos em questão por motivos simples, como a falta de colaboração ou perda de dados.

Neste trabalho, propõe-se uma medida metanalítica bayesiana obtida pela mistura das distribuições a posteriori do parâmetro de interesse proveniente de cada estudo individual, originando uma distribuição a posteriori combinada para o mesmo, chamada de distribuição a posteriori metanalítica. A medida metanalítica proposta é uma distribuição de probabilidade e não apenas uma 
simples medida de efeito ponderada. O foco principal está na metanálise caso a caso, uma vez que esta é considerada o padrão ouro da metanálise. No entanto, nos casos em que as estatísticas suficientes estiverem disponíveis, a metanálise caso a caso será equivalente à metanálise baseada na literatura.

O objetivo principal deste trabalho é apresentar uma nova medida metanalítica bayesiana útil a qualquer tipo problema relacionado à metanálise. A medida metanalítica proposta, por ser uma distribuição de probabilidade, é muito mais informativa que as medidas tradicionais, pois fornece informação sobre o comportamento do parâmetro em questão. Esta medida será útil também para encontrar similaridades entre os estudos, assim como uma análise de cluster e, consequentemente, diferenças entre os agrupamentos sugeridos pela análise. Embora essa medida seja útil a qualquer tipo de problema, será dada ênfase aos problemas que envolvem dados de sobrevivência. O software estatístico utilizado ao longo deste trabalho foi o $R$.

\subsection{Organização do trabalho}

Além desta introdução, a tese contém dois capítulos em que o tema comum é a investigação e a aplicação da metanálise caso a caso sob a perspectiva bayesiana. Cada capítulo possui uma introdução, onde é feita uma revisão bibliográfica de cada tema. Cada capítulo é independente dos demais, no sentido de que são introduzidas notações e comentários intrínsecos a cada um deles. No Capítulo 3, são citados resultados do Capítulo 2, mas a função de cada um desses capítulos é ser independente. Nesta tese, abordam-se algumas aplicações da metanálise proposta.

No Capítulo 2, é proposta uma medida metanalítica bayesiana baseada na mistura das distribuições a posteriori do parâmetro de interesse de cada estudo pertencente à metanálise. Ou seja, a medida metanalítica proposta é uma distribuição de probabilidade da característica de interesse e não apenas uma simples medida-resumo da mesma, acarretando em uma melhor conclusão do problema. O objetivo é trabalhar com a metanálise caso a caso, uma vez que esta é considerada o padrão ouro. Exemplos com dados reais de metanálises são apresentados e comparados com a metodologia usual, analisando algumas vantagens e desvantagens do método proposto.

No Capítulo 3, é aplicada a metodologia desenvolvida no Capítulo 2 para dados de sobrevivência completos e censurados. O objetivo é utilizar a distribuição a posteriori metanalítica dos parâmetros do modelo em questão para encontrar a curva de sobrevivência metanalítica. Alguns modelos estatísticos são utilizados para estimar as curvas de sobrevivência, com foco principal no modelo Weibull, por ser um modelo bastante flexível. São apresentadas duas aplicações com dados completos e censurados em que também é possível trabalhar com a metanálise baseada na literatura. Aplicações com modelos de risco competitivos e modelos de regressão com dados censurados também são apresentados e discutidos.

O Capítulo 4, destina-se às conclusões finais obtidas neste trabalho e sugestões de pesquisas futuras. 


\section{Capítulo 2}

\section{Distribuição metanalítica}

Resumo: Neste capítulo, propôs-se um método bayesiano para a realização de uma metanálise caso a caso. O procedimento baseou-se na mistura das distribuições a posteriori do parâmetro em questão de cada estudo percentente à metanálise produzindo, assim, uma distribuição a posteriori combinada como medida metanalítica. Além disso, é feita uma breve comparação entre o método proposto e alguns métodos utilizados em metanálise. Alguns exemplos com dados simulados e reais são apresentados e discutidos.

Palavras-chave: Inferência bayesiana, Metanálise

\subsection{Introdução}

O papel da metanálise é sumarizar estudos publicados sobre um problema específico, por meio da estatística; assim, cada dia é mais fundamental em razão do avanço da ciência e do crescimento do número de publicações. Revisões sistemáticas e metanálises são consideradas instrumentos importantes na avaliação de terapias médicas e úteis, quando há vontade de não se ignorar muitas das evidências disponíveis.

Glass [1976] define metanálise como uma análise de análises, ou seja, uma análise estatística que visa a combinar resultados já encontrados em análises anteriores de diferentes estudos de mesmo interesse. A metanálise objetiva combinar estudos realizados sob diferentes condições, com distintos níveis de precisão e por grupos de pesquisadores de diferentes regiões e formações. Assim, esperam-se conclusões mais amplas do que as obtidas pelos estudos que compõem a sistematização (Faggard et al. [1996]).

Quando uma metanálise é bem planejada e executada, é uma poderosa ferramenta para sínteses; trata-se de um método analítico, no qual todos os estudos são integrados e os seus resultados agrupados em uma única conclusão. Desse modo, quando a metanálise é comparada a outras formas de revisão de estudos individuais, apresenta a grande vantagem de sofrer menos influência pela opinião pessoal do pesquisador, fornecendo conclusões imparciais. Além disso, em uma metanálise todos os resultados provenientes dos estudos individuais examinados são relatados, e o leitor poderá facilmente recalculá-los e compará-los com as conclusões obtidas pelos autores (Leandro [2005]).

A metanálise baseada na literatura, por trabalhar apenas com os resultados dos estudos, pode nos levar a conclusões viesadas e, muitas vezes, equivocadas. Assim, é preferível o uso da metanálise caso a caso, uma vez que esta trabalha com os dados brutos de cada indivíduo; sendo este tipo mais informativo e possibilita qualquer tipo de análise de dados, incluindo análises com dados que dependam do tempo, fornecendo resultados mais precisos. Por outro lado, existem circunstâncias em que não se pode ter acesso aos dados dos estudos em questão por motivos simples, como a falta de colaboração ou perda de dados, impossibilitando o uso desta técnica (Higgins e Greens [2008]).

O objetivo deste capítulo é apresentar uma medida metanalítica bayesiana pautada na mistura 
das distribuições a posteriori do parâmetro de interesse de cada estudo pertencente à metanálise. Isto é, esta medida metanalítica proposta é uma distribuição de probabilidade da quantidade de interesse e não apenas uma simples medida-resumo da mesma, acarretando melhor conclusão do problema.

Este capítulo divide-se como se segue. A Seção 2 contém a metodologia para se produzir a medida metanalítica bayesiana proposta. A Seção 3 apresenta dois exemplos com dados de metanálises utilizando a metodologia proposta. O primeiro exemplo, utiliza a proporção como medida de efeito e o segundo, a diferença entre médias. Finalmente, na Seção 4 estão algumas considerações.

\subsection{Metodologia}

Visto que a distribuição a posteriori para a quantidade de interesse representa, sob a abordagem bayesiana, a mais completa inferência que se pode fazer a seu respeito, a proposta estatística deste trabalho para resolver problemas em metanálise é misturar as distribuições a posteriori dos parâmetros de interesse de cada estudo pertencente à metanálise. Como a operação bayesiana, muitas vezes, é de difícil execução, são utilizados métodos numéricos e o método aproximado de simulação de Monte Carlo via Cadeias de Markov (MCMC).

O paradigma bayesiano baseia-se na especificação de um modelo probabilístico para os dados observados $D$, dado um vetor de parâmetros desconhecidos $\boldsymbol{\theta}$, levando à função de verossimilhança $L(D, \boldsymbol{\theta})$. Assume-se que $\boldsymbol{\theta}$ é aleatório, com função a priori denotada por $\pi(\boldsymbol{\theta})$. A inferência sobre $\boldsymbol{\theta}$ é baseada na distribuição a posteriori, obtida via teorema de Bayes. A distribuição a posteriori de $\boldsymbol{\theta}$ é dada por:

$$
\pi(\boldsymbol{\theta} \mid D)=\frac{L(D, \boldsymbol{\theta}) \pi(\boldsymbol{\theta})}{\int_{\Theta} L(D, \boldsymbol{\theta}) \pi(\boldsymbol{\theta}) d \boldsymbol{\theta}},
$$

onde $\Theta$ denota o espaço paramétrico de $\boldsymbol{\theta}$. De $(2.1), \pi(\boldsymbol{\theta} \mid D)$ é proporcional à multiplicação da função de verossimilhança e da priori,

$$
\pi(\boldsymbol{\theta} \mid D) \propto L(D, \boldsymbol{\theta}) \pi(\boldsymbol{\theta})
$$

envolvendo a contribuição dos dados observados por meio de $L(D, \boldsymbol{\theta})$ e a contribuição da informação a priori quantificada por $\pi(\boldsymbol{\theta})$. A quantidade $m(D)=\int_{\Theta} L(D, \boldsymbol{\theta}) \pi(\boldsymbol{\theta}) d \boldsymbol{\theta}$ é a constante normalizadora de $\pi(\boldsymbol{\theta} \mid D)$ e é, frequentemente, chamada de distribuição marginal dos dados ou de distribuição preditiva (Ibrahim et al. [2005]).

Para simplificar e introduzir a ideia do método proposto, considere um exemplo fictício de uma metanálise de dois estudos.

Exemplo 1: Suponha que a variável de interesse seja normalmente distribuída com média $\mu$ e variância $\sigma^{2}$. Os dados do estudo 1 foram gerados de uma distribuição Normal com média -3 e variância $15, X \sim \operatorname{Normal}(-3 ; 15)$, e do estudo 2 , de uma distribuição Normal com média 3 e variância $12, Y \sim \operatorname{Normal}(3 ; 12)$. O tamanho amostral de ambos os estudos é igual a 30 $\left(n_{x}=n_{y}=30\right)$. A média e a variância amostral do estudo 1 são $\bar{x}=\sum_{i=1}^{n_{x}} x_{i} / n_{x}=-2,98$ e $s_{x}^{2}=\frac{\sum_{i=1}^{n_{x}}\left(x_{i}-\bar{x}\right)^{2}}{n_{x}-1}=18,04$ e do estudo 2 são, respectivamente, $\bar{y}=\sum_{i=1}^{n_{y}} y_{i} / n_{y}=3,35$ e $s_{y}^{2}=$ $\frac{\sum_{i=1}^{n_{y}}\left(y_{i}-\bar{y}\right)^{2}}{n_{y}-1}=8,37$. A função de verossimilhança para os estudos 1 e 2 são, respectivamente,

$$
L_{x}\left(\boldsymbol{x}, \mu, \sigma^{2}\right) \propto\left(\sigma^{2}\right)^{-n_{x} / 2} \exp \left\{-\frac{1}{2 \sigma^{2}}\left[\left(n_{x}-1\right) s_{x}^{2}+n_{x}(\mu-\bar{x})^{2}\right]\right\}
$$

e

$$
L_{y}\left(\boldsymbol{y}, \mu, \sigma^{2}\right) \propto\left(\sigma^{2}\right)^{-n_{y} / 2} \exp \left\{-\frac{1}{2 \sigma^{2}}\left[\left(n_{y}-1\right) s_{y}^{2}+n_{y}(\mu-\bar{y})^{2}\right]\right\} .
$$

Considerando a priori de Jeffreys para $\left(\mu, \sigma^{2}\right), \pi\left(\mu, \sigma^{2}\right) \propto\left(\sigma^{2}\right)^{-1}$, e a função de verossimilhança em (2.3) e (2.4), as distribuições conjuntas a posteriori de $\mu$ e $\sigma^{2}$ em ambos os estudos seguem 
distribuição Normal-Gama-Inversa, dadas por:

$$
\pi\left(\mu, \sigma^{2} \mid \boldsymbol{x}\right)=\frac{\sqrt{n_{x}}}{\sigma \sqrt{2 \pi}} \frac{\left[\left(n_{x}-1\right) s_{x}^{2} / 2\right]^{n_{x} / 2}}{\Gamma\left(n_{x} / 2\right)}\left(\frac{1}{\sigma^{2}}\right)^{\frac{n_{x}+1}{2}+1} \exp \left\{-\frac{\left(n_{x}-1\right) s_{x}^{2}+n_{x}(\mu-\bar{x})^{2}}{2 \sigma^{2}}\right\}
$$

e

$$
\pi\left(\mu, \sigma^{2} \mid \boldsymbol{y}\right)=\frac{\sqrt{n_{y}}}{\sigma \sqrt{2 \pi}} \frac{\left[\left(n_{y}-1\right) s_{y}^{2} / 2\right]^{n_{y} / 2}}{\Gamma\left(n_{y} / 2\right)}\left(\frac{1}{\sigma^{2}}\right)^{\frac{n_{y}+1}{2}+1} \exp \left\{-\frac{\left(n_{y}-1\right) s_{y}^{2}+n_{y}(\mu-\bar{y})^{2}}{2 \sigma^{2}}\right\} .
$$

Ou seja, $\left(\mu, \sigma^{2} \mid \boldsymbol{x}\right) \sim N I G\left(\bar{x}, n_{x}, \frac{n_{x}-1}{2}, \frac{\left(n_{x}-1\right) s_{x}^{2}}{2}\right)$ e $\left(\mu, \sigma^{2} \mid \boldsymbol{y}\right) \sim N I G\left(\bar{y}, n_{y}, \frac{n_{y}-1}{2}, \frac{\left(n_{y}-1\right) s_{y}^{2}}{2}\right)$. Os valores esperados a posteriori e a matriz de variância-covariância a posteriori de $\mu$ e $\sigma^{2}$ para (2.5) são, respectivamente,

$$
E\left[\mu, \sigma^{2} \mid \boldsymbol{x}\right]=\left(\begin{array}{c}
\bar{x} \\
\frac{\left(n_{x}-1\right) s_{x}^{2}}{\left(n_{x}-3\right)}
\end{array}\right), n_{x}>3
$$

$\mathrm{e}$

$$
\operatorname{Cov}\left[\mu, \sigma^{2} \mid \boldsymbol{x}\right]=\left[\begin{array}{cc}
\frac{s_{x}^{2}\left(n_{x}-1\right)}{n_{x}\left(n_{x}-3\right)} & 0 \\
0 & \frac{2 s_{x}^{2}\left(n_{x}-1\right)}{\left(n_{x}-5\right)\left(n_{x}-3\right)^{2}}
\end{array}\right], n_{x}>5
$$

Os valores esperados a posteriori e a matriz de variância-covariância de $\mu$ e $\sigma^{2}$ para o modelo (2.6) são análogos a (2.7) e (2.8). Vale ressaltar que a distribuição a posteriori conjunta de $\mu$ e $\sigma^{2}$ depende dos dados apenas por meio das estatísticas suficientes $\left(n_{x}, \bar{x}, s_{x}^{2}\right)$ e $\left(n_{y}, \bar{y}, s_{y}^{2}\right)$, ou seja, a evidência fornecida pela estatística suficiente é idêntica à fornecida pelos dados (Paulino et al. [2003]). Neste caso, realizar uma metanálise baseada na literatura é equivalente a realizar uma metanálise caso a caso, sem perda de qualidade.

Utilizando (2.5) e (2.6), sugere-se misturar as distribuições a posteriori de $\left(\mu, \sigma^{2}\right)$ de cada estudo. Isto é,

$$
\pi_{M}\left(\mu, \sigma^{2} \mid \boldsymbol{x}, \boldsymbol{y}\right)=\frac{n_{x} \pi\left(\mu, \sigma^{2} \mid \boldsymbol{x}\right)+n_{y} \pi\left(\mu, \sigma^{2} \mid \boldsymbol{y}\right)}{n_{x}+n_{y}} .
$$

Na Figura 2.1(a), estão as curvas de nível das distribuições a posteriori conjunta de $\left(\mu, \sigma^{2}\right)$ para cada estudo, a fim de observar os gráficos tridimensionais em apenas duas dimensões. Observa-se que a diferença entre as médias a posteriori parece grande. Na Figura 2.1(b), estão as curvas de nível da distribuição a posteriori metanalítica conjunta de $\left(\mu, \sigma^{2}\right)$, que é dada pela mistura das distribuições a posteriori de $\left(\mu, \sigma^{2}\right)$ dos dois estudos em questão e, na Figura 2.1(c), está o gráfico tridimensional da distribuição a posteriori metanalítica dada em (2.9). Ou seja, a medida metanalítica proposta é uma distribuição de $\left(\mu, \sigma^{2}\right)$ e não apenas uma simples medida-resumo. Neste caso, a medida metanalítica de $\left(\mu, \sigma^{2}\right)$ é uma distribuição bimodal, sugerindo a existência de dois grupos, sendo necessário uma melhor investigação do problema. Vale ressaltar que as regiões de maior densidade nos gráficos são aquelas mais prováveis aos "verdadeiros"parâmetros. A estimação por região HPD (Highest Posterior Density) com 95\% de credibilidade está na Figura 2.1(d). O HPD é a menor região/ intervalo que contém o verdadeiro valor do parâmetro de interesse, com probabilidade maior ou igual a 95\% (Paulino et al. [2003]). 
(a)

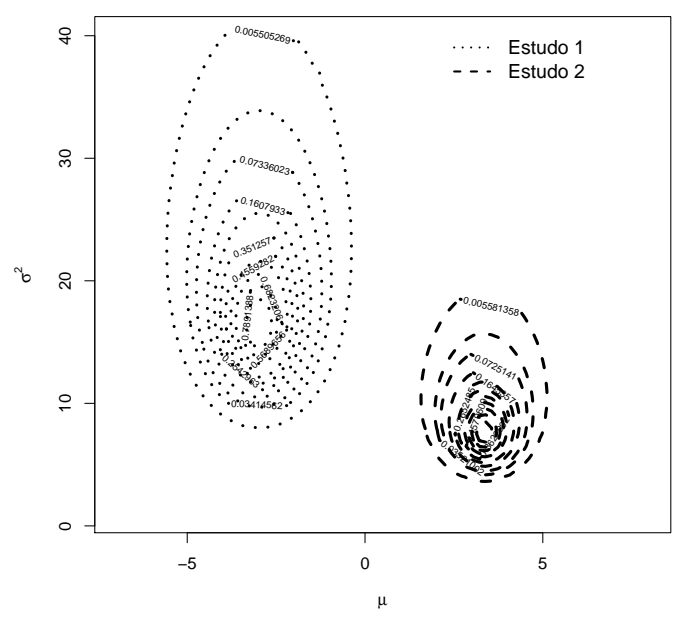

(c)

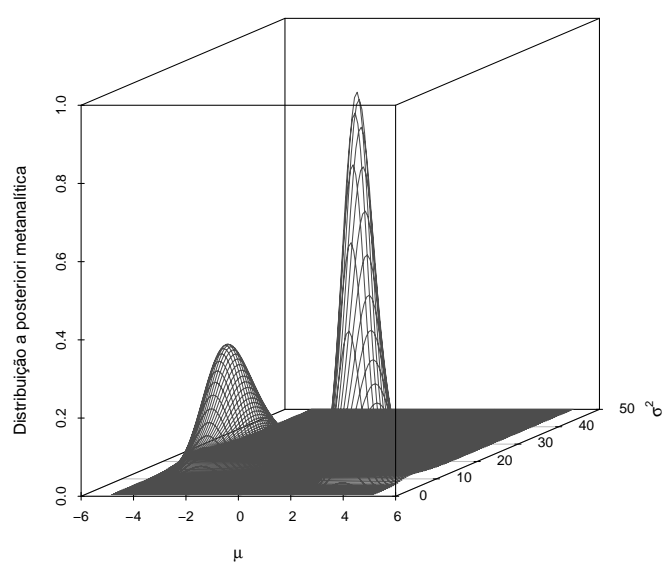

(b)

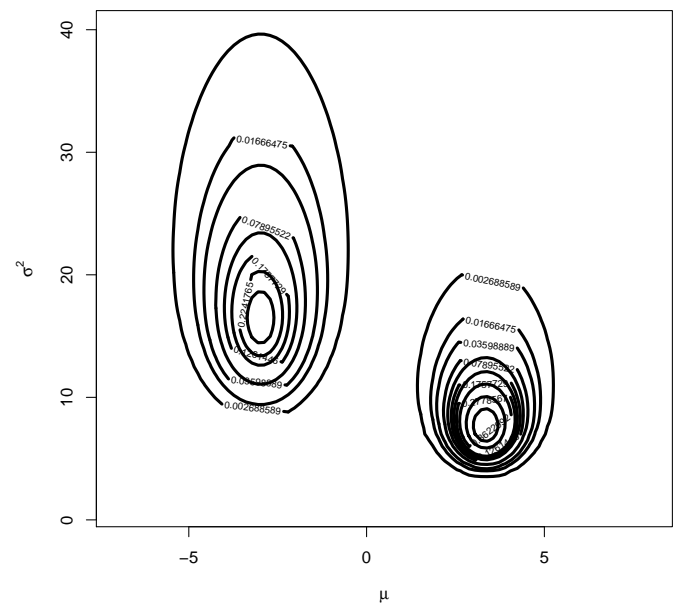

(d)

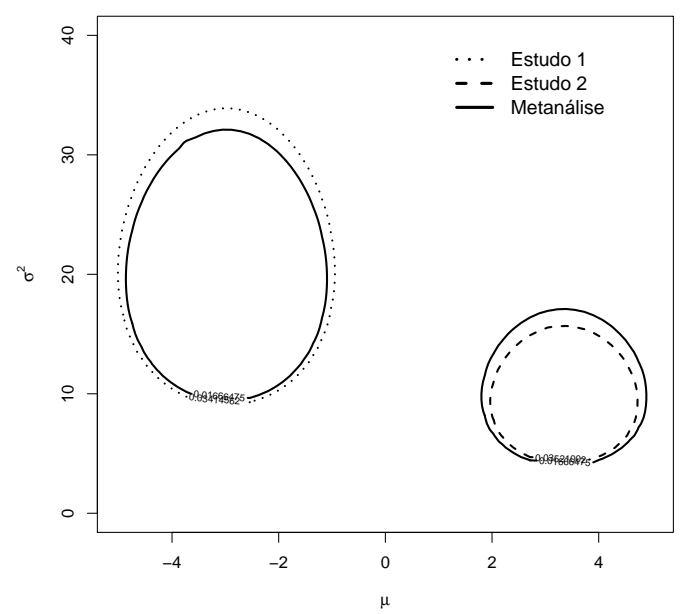

Figura 2.1: (a) Curvas de nivel da distribuição conjunta a posteriori de $\left(\mu, \sigma^{2}\right)$ em cada estudo. (b) Curvas de nivel da distribuição a posteriori metanalítica de $\left(\mu, \sigma^{2}\right)$. (c) Gráfico tridimensional da distribuição a posteriori metanalitica de $\left(\mu, \sigma^{2}\right)$. (d) Região HPD de $95 \%$ de credibilidade para $\left(\mu, \sigma^{2}\right)$.

Se a medida metanalítica fosse calculada pelo método de DerSimonian e Laird, esta seria igual a $0,21 \mathrm{com}$ intervalo de $95 \%$ de confiança igual a $[-5,99 ; 6,41]$. Os resultados dessa metanálise baseada na literatura estão resumidos no gráfico Forest Plot, Figura 2.2 (Lewis e Clarke [2001]). Uma breve revisão do método, para melhor entendimento, é apresentada mais adiante e o estimador metanalítico é dado em (2.10). No entanto, pergunta-se: isto faz sentido? Fazendo uma metáfora, o resultado encontrado é o mesmo que dizer que, estar com a cabeça no congelador e com os pés no forno, em média, a temperatura está agradável. 


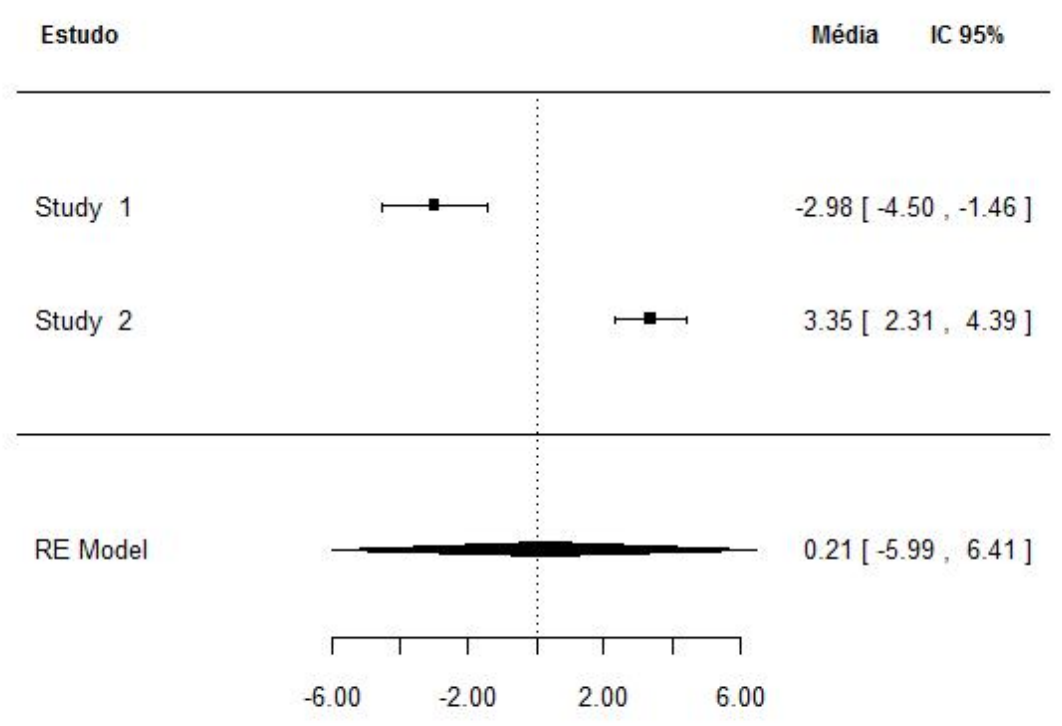

Figura 2.2: Forest Plot para os dados do Exemplo 1.

Para se tentar aproximar os métodos para eventuais comparações, encontra-se a distribuição a posteriori metanalítica apenas em função de $\mu$. As distribuições a posteriori marginal de $\mu$, nos estudos 1 e 2 , são $\mu \mid \boldsymbol{x} \sim t_{n_{x}-1}\left(\bar{x}, s_{x}^{2} / n_{x}\right)$ e $\mu \mid \boldsymbol{y} \sim t_{n_{y}-1}\left(\bar{y}, s_{y}^{2} / n_{y}\right)$ (Lee [2012]). Para se encontrar a medida metanalítica de $\mu$ utiliza-se (2.9) (Figura 2.3(a)). É importante notar que a distribuição a posteriori metanalítica marginal de $\mu$ também é uma distribuição bimodal com intervalo HPD de $95 \%$ de credibilidade dado pelos intervalos disjuntos $[-4,55 ;-1,41]$ e $[2,17 ; 4,53]$. Também se pode observar que o valor metanalítico encontrado pelo método de DerSimonian e Laird está fora dos intervalos HPD. Neste caso, a distribuição metanalítica é bimodal e encontrar a média a posteriori de $\mu$ não traz muita informação. A medida proposta sugere a existência de dois grupos distintos, que nos leva a pensar melhor sobre o problema e na possível combinação desses estudos.

Suponha, ainda, que estejam disponíveis apenas o resultado da metanálise usual, o tradicional diamante do Forest Plot, e o resultado da metanálise proposta, a distribuição a posteriori metanalítica (Figura 2.3(b)). Se apenas estes resultados estiverem disponíveis, fica claro que o resultado obtido pelo método proposto permite conclusões mais abrangentes e é mais informativo que o usual. Portanto, utilizar uma distribuição de probabilidade como medida metanalítica é mais plausível, pois, pode-se observar o comportamento do parâmetro em questão. Vale ressaltar que, neste exemplo, existe também a vantagem que os resultados obtidos pelo método proposto, considerando as estatísticas suficientes, são os mesmos obtidos pela metanálise caso a caso. Uma observação importante é que esta medida também pode encontrar similaridades entre os estudos e diferenças entre os grupos sugeridos por esta distribuição, que pode ser causada por algum fator não investigado, mostrando uma vantagem quando se utiliza esta medida. É importante deixar claro que o objetivo não é a comparação dos métodos, uma vez que os mesmos não são comparáveis. No entanto, o intuito é evidenciar possíveis falhas de uma metodologia, que é amplamente utilizada na metanálise e chamar a atenção para a forma como o mesmo problema pode ser resolvido. 
(a)

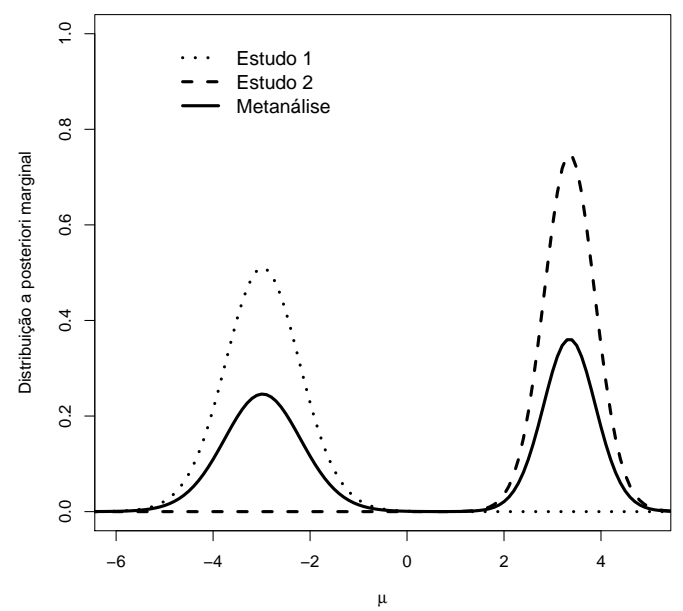

(b)

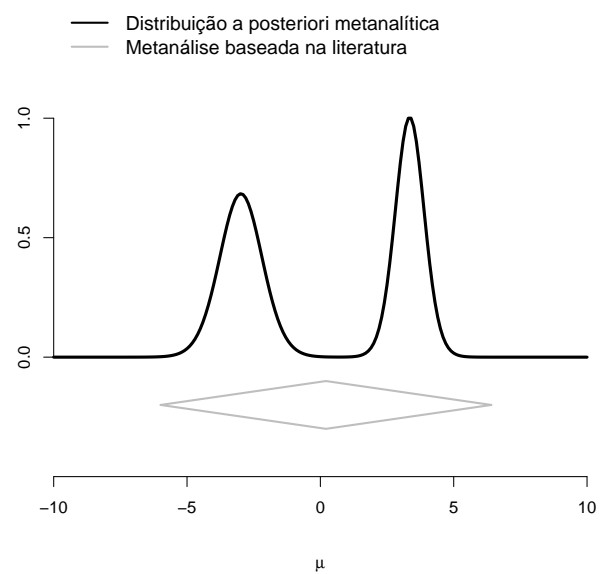

Figura 2.3: (a) Distribuições a posteriori marginal de $\mu$. (b) Resultados da metanálise proposta (distribuição a posteriori metanalítica padronizada) e da metanálise usual (diamante).

O método de DerSimonian e Laird nada mais é que uma média ponderada das medidas de efeito dos estudos. No método, não há a suposição de homogeneidade entre os estudos. A medida metanalítica é dada por:

$$
\hat{\theta}_{M D L}=\frac{\sum_{j=1}^{J} w_{j}^{*} \hat{\theta}_{j}}{\sum_{j=1}^{J} w_{j}^{*}},
$$

em que $w_{j}^{*}=\frac{1}{\tau^{2}+w_{j}^{-1}}, w_{j}=\frac{1}{\hat{\sigma}_{j}^{2}}, \hat{\tau^{2}}=\frac{Q-(J-1)}{\sum_{j=1}^{J} w_{j}-\frac{\sum_{j=1}^{J} w_{j}^{2}}{\sum_{j=1}^{J} w_{j}}}, Q=\sum_{j=1}^{J} w_{j}\left(\hat{\theta}_{j}-\hat{\theta}_{M}\right)^{2}, \hat{\theta}_{M}=\frac{\sum w_{j} \hat{\theta}_{j}}{\sum w_{j}}, \hat{\theta}_{j}$ é a medida de efeito do $j$-ésimo estudo e $\tau$ é a variabilidade estimada entre os estudos, $j=1,2$. $\hat{\tau^{2}}$ pode ser menor que zero e, se isto acontecer, $\hat{\tau^{2}}=0$ e a medida metanalítica é a mesma que a calculada pelo método de Mantel-Haenszel. O intervalo de $95 \%$ de confiança para $\hat{\theta}_{M D L}$ é dado por $\left[\hat{\theta}_{M D L} \pm 2 / \sqrt{\sum_{j=1}^{J} w^{*}}\right]$ (Mazin e Martinez [2009]).

Outro aspecto importante na metanálise a ser ressaltado é que esta não deve ser confundida com análise combinada. A análise combinada de dados desconsidera o fator estudo, unindo todos os indivíduos em uma única amostra e considerar uma metanálise, como se fosse um grande estudo, é perder toda a variabilidade existente entre os mesmos. A comparação entre a análise combinada e a metanálise, para os dados deste exemplo, pode ser vista na Figura 2.4. Ignorando, então, o fato de que os dados provêm de estudos diferentes, assume-se uma única amostra de tamanho 60 . Dessa amostra única, obtém-se a média igual a 0,18 e o desvio-padrão de 4,81. Considerando a abordagem bayesiana descrita anteriormente, são econtradas a distribuição a posteriori conjunta de $\left(\mu, \sigma^{2}\right)$ como em (2.5) e a distribuição a posteriori marginal de $\mu$, representadas na Figura 2.4(a) e na Figura 2.4(b). A média a posteriori de $\mu$ é 0,18 com intervalo HPD de $95 \%$ de credibilidade igual a $[-1,06 ; 1,42]$. Nota-se que a análise combinada difere consideravelmente da metanálise proposta o que não ocorre com a metanálise via método de DerSimonian e Laird. 
(a)

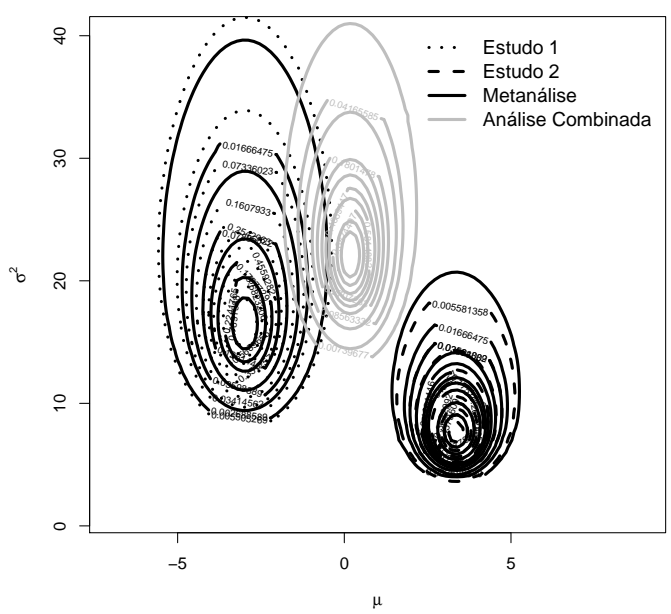

(b)

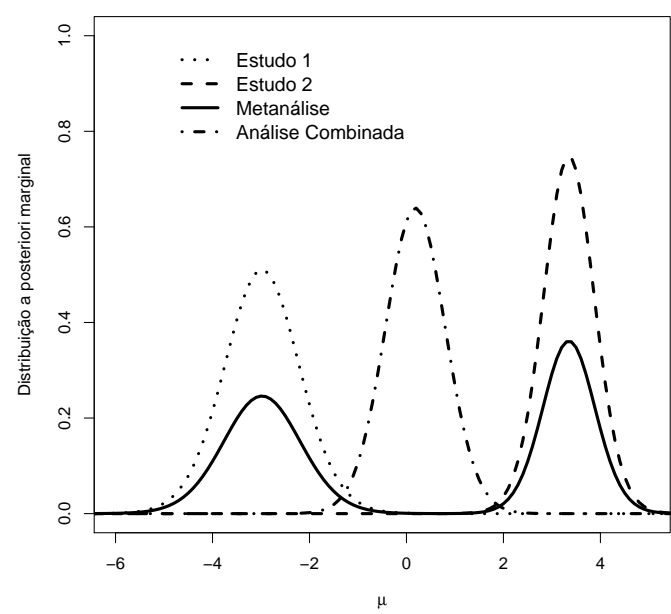

Figura 2.4: (a) Curvas de nível da distribuição a posteriori conjunta de $\mu$ e $\sigma^{2}$. (b) Distruibuição a posteriori marginal de $\mu$.

\subsubsection{Generalização do método proposto}

Considere uma metanálise de $J$ estudos independentes que investigam certa característica de interesse $\theta$. Para o $j$-ésimo estudo $(j=1, \ldots J)$, suponha que $\mathbf{X}_{j}=\left(X_{1, j}, X_{2, j}, \ldots, X_{n_{j}, j}\right)^{T}$ denota um vetor de variáveis aleatórias de tamanho $n_{j}$, com função densidade de probabilidade $f\left(\boldsymbol{x}_{j}, \theta\right)$ no espaço de probabilidade $(\mathcal{X}, \mathcal{F}, \mathcal{P})$. Considerando $\boldsymbol{x}_{j}=\left(x_{1, j}, x_{2, j}, \ldots, x_{n_{j}, j}\right)$ um vetor aleatório observado de $\mathbf{X}_{j}$, tem-se que a função de verossimilhança para o $j$-ésimo estudo é dada por $L_{j}\left(\boldsymbol{x}_{j}, \theta\right)=\prod_{i=1}^{n_{j}} f\left(x_{i j}, \theta\right)$. Admitindo a metodologia bayesiana, observa-se, respectivamente, a função a priori de $\theta$ e a distribuição a posteriori de $\theta, \pi(\theta)$ e $\pi_{j}\left(\theta \mid \boldsymbol{x}_{j}\right) \propto L_{j}\left(\boldsymbol{x}_{j}, \theta\right) \pi(\theta)$. Em geral, a função a priori é a mesma para todos os estudos, pois independe de onde o experimento foi conduzido. Então, a medida metanalítica proposta é a mistura das $J$ distribuições a posteriori de $\theta$, produzindo uma única distribuição para $\theta$, que é chamada de distribuição a posteriori metanalítica de $\theta$. A mistura de densidades é uma técnica utilizada em alguns casos para derivar conclusões a respeito de $\theta$ (O'Hagan [1991]). Portanto, a distribuição a posteriori metanalítica de $\theta$ é dada por

$$
\pi_{M}(\theta \mid \boldsymbol{x})=\sum_{j=1}^{J} \omega_{j} \pi_{j}\left(\theta \mid \boldsymbol{x}_{j}\right) \propto\left[\sum_{j=1}^{J} \omega_{j} L_{j}\left(\boldsymbol{x}_{j}, \theta\right)\right] \pi(\theta),
$$

em que $\omega_{j}$ é o peso do $j$-ésimo estudo. Estes pesos são fixos e podem representar a importância dos estudos, o tamanho amostral, etc. Inicialmente, considere $\omega_{j}=\frac{n_{j}}{\sum_{j=1}^{J} n_{j}}$. A média a posteriori metanalítica e a variância a posteriori metanalítica para $\theta$ são, respectivamente,

$$
E_{M}(\theta \mid \boldsymbol{x})=\sum_{j=1}^{J} \omega_{j} E_{j}\left[\theta \mid \boldsymbol{x}_{j}\right]
$$

e

$$
\operatorname{Var}_{M}(\theta \mid \boldsymbol{x})=\sum_{j=1}^{J} \omega_{j}^{2} \operatorname{Var}_{j}\left[\theta \mid \boldsymbol{x}_{j}\right]
$$

A medida metanalítica proposta é uma medida geral, isto é, qualquer função a priori e qualquer função de verossimilhança podem ser utilizadas em (2.11). O que faz com que esta possa ser usada, 
desde o problema mais trivial de metanálise aos mais complexos. Vale ressaltar também que, quando a estatística suficiente para $\theta$ estiver disponível, realizar uma metanálise baseada na literatura será equivalente a realizar uma metanálise caso a caso, sem perda de informação.

É importante deixar claro, para que se tenha uma metanálise de qualidade, além de uma técnica estatística apropriada, é importante que os estudos a serem combinados tenham sido avaliados com cuidado, para que haja confiança em sua associação. Portanto, é necessário que critérios de seleção de estudos sejam estabelecidos (Justo et al. [2005]).

Para Egger e Smith [1997], a metanálise exprime um planejamento cauteloso. A definição a priori de critérios qualificados para os estudos é incluída em uma busca detalhada, de modo a garantir uma alta qualidade da metanálise. Existem diferentes métodos estatísticos para a combinação dos dados; no entanto, não há um único "método correto". Embora os estudos pertencentes à metanálise envolvam o mesmo assunto, existem diferenças entre eles, que é denominada heterogeneidade entre os estudos, esta é um dos problemas usuais em metanálise e, uma solução é incorporá-la à análise estatística. Os modelos estatísticos que incorporam o componente de variabilidade entre os estudos são amplamente discutidos, tanto na teoria clássica como na bayesiana. Berry [1989] considera a formulação de um modelo hierárquico bayesiano para descrever e explicar a heterogeneidade existente entre os estudos individuais, e a teoria clássica envolve a combinação da magnitude dos efeitos (DerSimonian e Laird [1986]). Outro modelo que vem sendo empregado para analisar a heterogeneidade existente entre os estudos, é o modelo de mistura finita sugerido por Schlattmann [2009].

Os métodos bayesianos são mais flexíveis que os clássicos. Os dados afetam a inferência bayesiana apenas pela função de verossimilhança, ou seja, a regra de parada e outras características do desenho do estudo são irrelevantes. Tais métodos também oferecem uma estrutura conveniente para a incorporação das informações disponíveis a priori e são aplicáveis a problemas que combinam estudos com tamanhos amostrais pequenos, quando os estimadores bayesianos são mais precisos que os obtidos via máxima verossimilhança (Pissini [2006]). Um aspecto importante a ser ressaltado é que o método proposto neste trabalho não discute a questão de heterogeneidade ou homogeneidade entre os estudos, uma vez que sempre se leva em consideração a variabilidade existente entre os estudos incluídos na metanálise.

A seguir, discute-se o uso de alguns modelos estatísticos utilizados em metanálise por intermédio de um exemplo com respostas dicotômicas apresentado por Berry [1989].

Exemplo 2: Considere os resultados de uma metanálise composta de nove estudos, envolvendo a droga antidepressiva SAMe. Os dados da Tabela 2.1 contêm os resultados para os nove estudos, com o número de pacientes no $j$-ésimo estudo dado por $n_{j}$ e o número de sucessos dado por $y_{j}$.

Tabela 2.1: Sucessos observados no uso da droga antidepressiva SAMe.

\begin{tabular}{cccc}
\hline$j$ & $y_{j}$ & $n_{j}$ & $\hat{p}_{j}=y_{j} / n_{j}$ \\
\hline 1 & 20 & 20 & 1,00 \\
2 & 4 & 10 & 0,40 \\
3 & 11 & 16 & 0,69 \\
4 & 10 & 19 & 0,53 \\
5 & 5 & 14 & 0,36 \\
6 & 36 & 46 & 0,78 \\
7 & 9 & 10 & 0,90 \\
8 & 7 & 9 & 0,78 \\
9 & 4 & 6 & 0,67 \\
\hline Total & 106 & 150 & 0,71 \\
\hline
\end{tabular}

A variável aleatória de interesse $X$ representa o sucesso no uso da droga antidepressiva, ou seja, $X=1$ se ocorreu o sucesso no uso da droga com probabilidade $p$ ou $X=0$ se não ocorreu o sucesso no uso da droga, com probabilidade $1-p$. Então, $X \mid p \sim \operatorname{Bernouli}(p)$ e sua distribuição 
de probabilidade é

$$
P(X=x \mid p)=p^{x}(1-p)^{1-x} .
$$

Logo, para uma amostra aleatória de $X$ com tamanho $n$, o número de tentativas que resulta em sucesso, $Y=\sum_{i=1}^{n} x_{i}$, segue distribuição Binomial com parâmetros $n$ e $p$.

Os resultados obtidos por meio da metanálise proposta por DerSimonian e Laird [1986] encontram-se resumidos no Forest Plot (Figura 2.5). A medida metanalítica encontrada é igual a 0,71 com intervalo de $95 \%$ de confiança, igual a $[0,53 ; 0,87]$. Portanto, a proporção combinada de sucessos no uso da droga é de 0,71 e se este experimento fosse repetido 100 vezes, seria esperado que em 95 vezes o verdadeiro valor do parâmetro estivesse entre 0,53 e 0,87. Esta conclusão é curiosa, pois três das proporções observadas encontram-se fora desse intervalo de confiança. Os resultados desse método são muito próximos dos encontrados se tais dados fossem considerados como uma única amostra na análise combinada (Figura 2.6). Uma análise combinada assume que todos os 150 pacientes são permutáveis, tal que os nove $p_{j}$ 's são iguais. A função de verossimilhança de $p$ é dada por:

$$
L(\boldsymbol{y}, p)=p^{106}(1-p)^{44}
$$

A Figura 2.6 mostra ser muito provável que $p$ esteja entre $60 \%$ e $80 \%$. O que também é curioso, visto que cinco das proporções observadas estão fora desse intervalo.

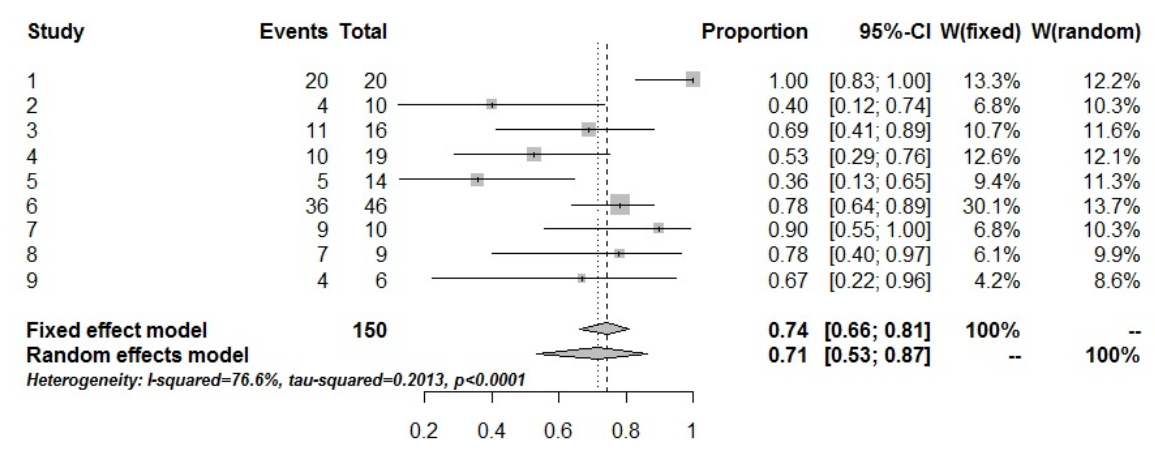

Figura 2.5: Forest Plot para a proporção de sucessos no uso da droga SAMe. 


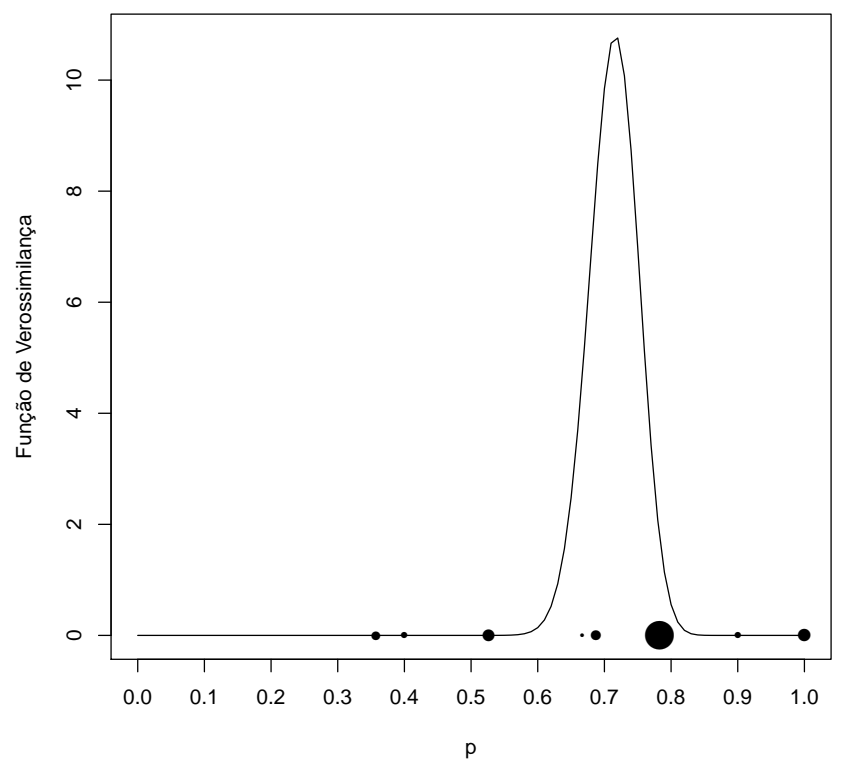

Figura 2.6: Análise combinada para a proporção de sucessos. (Os nove pontos correspondem às proporções observadas para os nove estudos, com as áreas dos pontos proporcionais aos tamanhos amostrais.)

A perspectiva bayesiana hierárquica considera que cada proporção de sucesso de cada estudo seja selecionada da mesma população. Quantificar a informação sobre a população requer uma distribuição de probabilidade da distribuição populacional (Berry [1989]).

Como dito anteriormente, a variável aleatória $Y_{j}$ é o número de sucessos entre os $n_{j}$ pacientes do estudo $j$, tal que, $y_{j} \sim \operatorname{Binomial}\left(n_{j}, p_{j}\right)$ para $j=1, \ldots, 9$. Então, a função de verossimilhança para $\boldsymbol{p}=\left(p_{1}, \ldots, p_{9}\right)^{T}$ é

$$
L(\boldsymbol{y}, \boldsymbol{p}) \propto \prod_{j=1}^{9} p_{j}^{y_{j}}\left(1-p_{j}\right)^{n_{j}-y_{j}} .
$$

Suponha que $p_{1}, \ldots, p_{9}$ seja uma amostra aleatória da distribuição populacional $F$, que é aleatória. Assuma que $F$ seja uma distribuição $\operatorname{Beta}(a, b)$, onde $a$ e $b$ são desconhecidos, de modo que a função de densidade de $p_{j}$ é

$$
\pi\left(p_{j} \mid a, b\right)=B(a, b) p_{j}^{a-1}\left(1-p_{j}\right)^{b-1},
$$

onde $a>0$ e $b>0$ e $B^{-1}(a, b)=\int_{0}^{1} p_{j}^{a-1}\left(1-p_{j}\right)^{b-1} d p_{j}$.

Como tudo que é desconhecido na abordagem bayesiana, deve-se assumir uma distribuição de probabilidade para $a$ e para $b, \pi(a, b)$. Neste exemplo, uma priori Uniforme é considerada,

$$
\pi(a, b) \propto 1 .
$$

Logo, a distribuição a posteriori de $(a, b, \boldsymbol{p})$ dado $\boldsymbol{y}$ é

$$
\pi(a, b, \boldsymbol{p} \mid \boldsymbol{y}) \propto \prod_{j=1}^{9} p_{j}^{y_{j}}\left(1-p_{j}\right)^{n_{j}-y_{j}} B(a, b) p_{j}^{a-1}\left(1-p_{j}\right)^{b-1}
$$

e a distribuição a posteriori condicional de $\boldsymbol{p}$ é

$$
\pi(\boldsymbol{p} \mid a, b, \boldsymbol{y}) \propto \prod_{j=1}^{9} p_{j}^{a+y_{j}-1}\left(1-p_{j}\right)^{b+n_{j}-y_{j}-1} .
$$


A Figura 2.7 mostra a densidade estimada a posteriori de $\boldsymbol{p}$ com média igual a 0,67 , moda igual a 0,75 e intervalo HPD de $95 \%$ de credibilidade igual a $[0,35 ; 0,99]$.

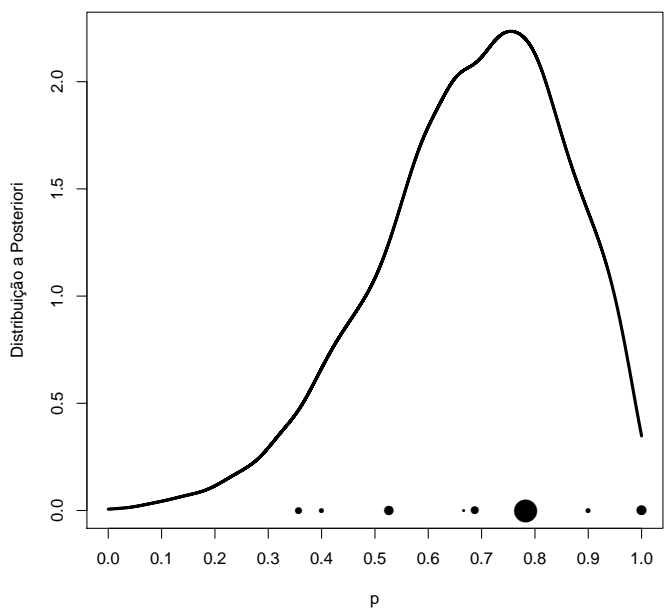

Figura 2.7: Densidade a posteriori estimada da proporção dos sucessos, considerando priori Uniforme (2.18).Como na Figura 2.6, os pontos correspondem às proporções observadas.

Outra maneira possível de se resolver o problema é por meio de um modelo de mistura finita (McLachlan e Peel [2000]). Então, considere uma amostra $x_{1}, x_{2}, \ldots, x_{N} \operatorname{com} x_{i} \stackrel{\text { iid }}{\sim} f(x, P) i=$ $1, \ldots, N$, e

$$
f(x, \boldsymbol{P})=\sum_{j=1}^{g} w_{j} f\left(x, \theta_{j}\right)
$$

onde $w_{j}$ são os pesos da mistura, $\theta_{j}$ são os parâmetros dos componentes, $g$ é o número de componentes da mistura e $f(\bullet)$ é uma densidade. A estimação dos parâmetros da distribuição de mistura $\boldsymbol{P}$ é, predominantemente, feita por meio da função de verossimilhança. O interesse está nas estimativas de máxima verossimilhança de $\boldsymbol{P}$ que é dada pela maximização do log da função de verossimilhança,

$$
l(\boldsymbol{P})=\log L(x, \boldsymbol{P})=\sum_{i=1}^{N} \log \sum_{j=1}^{g} w_{j} f\left(x_{i}, \theta_{j}\right) .
$$

O modelo de mistura (2.21) é ajustado com $x_{i} \stackrel{\text { iid }}{\sim} \operatorname{Bernoulli}(p), i=1, \ldots, 149$, e $j=1,2, \ldots, 6$, para os dados em questão. Nesta abordagem são considerados todos os dados, como uma única amostra com o objetivo de se identificar o número $j$ dos componentes da mistura. Parte dos resultados encontrados, encontra-se na Tabela 2.2. Observa-se que o modelo de mistura finita com duas componentes é equivalente ao modelo de mistura finita com uma única componente. O resultado do modelo com seis componentes é curioso, pois, 4,7\% dos estudos, o que representa menos de um estudo, possuem sucesso no uso da droga de $25 \%$. Logo, o modelo é de difícil interpretação e poderá levar a conclusões não muito plausíveis. Comentários a parte, pelo critério de informação bayesiano (BIC), o melhor modelo de mistura é aquele com uma única componente (Schwarz [1978]). Em outras palavras, o modelo de mistura conclui que os estudos são homogêneos e que o percentual de sucesso no uso da droga, considerando todos os estudos, é igual a $70 \%$. 
Tabela 2.2: Ajuste do modelo de mistura finita para os dados da Tabela 2.1.

\begin{tabular}{ccccc}
\hline Componentes & Peso & Parâmetro & Log-verossimilhança & BIC \\
\hline 1 & 1 & 0,7066 & $-90,5303$ & 186,0712 \\
\hline 1 & 0,30 & 0 & & \\
2 & 0,70 & 1 & $-90,5318$ & 196,0955 \\
\hline 1 & 0,047 & 0,25 & & \\
2 & 0,111 & 0,37 & & \\
3 & 0,111 & 0,50 & & \\
4 & 0,111 & 0,62 & & \\
5 & 0,111 & 0,75 & & \\
6 & 0,509 & 0,87 & $-90,5303$ & 236,1776 \\
\hline
\end{tabular}

Por fim, a medida metanalítica é calculada pela metodologia proposta em (2.11). Então, para o estudo $j(j=1,2, \ldots, 9)$, suponha que $\mathbf{X}_{j}$ são variáveis aleatórias, condicionalmente independentes dado $p$. Considere $\boldsymbol{x}_{j}=\left(x_{1}, x_{2}, \ldots, x_{n_{j}}\right)^{T}$ uma amostra aleatória observada das variáveis $\mathbf{X}_{j}$. A função de verossimilhança para o $j$-ésimo estudo é

$$
L_{j}\left(\boldsymbol{x}_{j}, p\right)=\prod_{i=1}^{n_{j}} p^{x_{i j}}(1-p)^{1-x_{i j}}=p^{y_{j}}(1-p)^{n_{j}-y_{j}},
$$

sendo $y_{j}=\sum_{i=1}^{n_{j}} x_{i j}$.

Ao se considerar que a distribuição a priori de $p$ é Uniforme $(0,1)$ e a função de verossimilhança em (2.23), a distribuição a posteriori de $p$, para o $j$-ésimo estudo, é

$$
\pi_{j}\left(p \mid \boldsymbol{y}_{j}\right)=\frac{\Gamma\left(n_{j}+2\right)}{\Gamma\left(y_{j}+1\right) \Gamma\left(n_{j}-y_{j}+1\right)} p^{y_{j}}(1-p)^{n_{j}-y_{j}} .
$$

Ou seja, $p \mid \boldsymbol{y}_{j} \sim \operatorname{Beta}\left(y_{j}+1, n_{j}-y_{j}+1\right), j=1, \ldots, 9$. Encontrada a distribuição a posteriori de $p$ para cada estudo, o próximo passo será a obtenção da distribuição metanalítica de $p$.

Com base em (2.11) e considerando $\omega_{j}=\frac{n_{j}}{n} \operatorname{com} n=\sum_{j=1}^{9} n_{j}$, a distribuição a posteriori metanalítica de $p, \pi_{M}(p \mid \boldsymbol{y})$ é

$$
\pi_{M}(p \mid \boldsymbol{y})=\sum_{j=1}^{9}\left(\frac{n_{j}}{n}\right) \frac{\Gamma\left(n_{j}+2\right)}{\Gamma\left(y_{j}+1\right) \Gamma\left(n_{j}-y_{j}+1\right)} p^{y_{j}}(1-p)^{n_{j}-y_{j}} .
$$

Na Figura 2.8(a), estão as distribuições a posteriori de $p$ para cada estudo pertencente à metanálise e na Figura 2.8(b) está a distribuição metanalítica de $p$, dada pela mistura das distribuições a posteriori de $p$ dos nove estudos em questão. Nota-se que o comportamento da distribuição a posteriori metanalítica de $p$ não é simétrico. Algumas medidas-resumo a posteriori para a distribuição metanalítica são calculadas como, mediana $(0,72)$, moda $(0,78)$, média $(0,69)$ e intervalo HPD de $95 \%$ de credibilidade, $[0,31 ; 1,00]$. A distribuição a posteriori metanalítica de $p$ sugere a existência de uma distribuição trimodal, de modo que, além de $0,78,0,5$ e 1, também são máximos locais. 
(a)

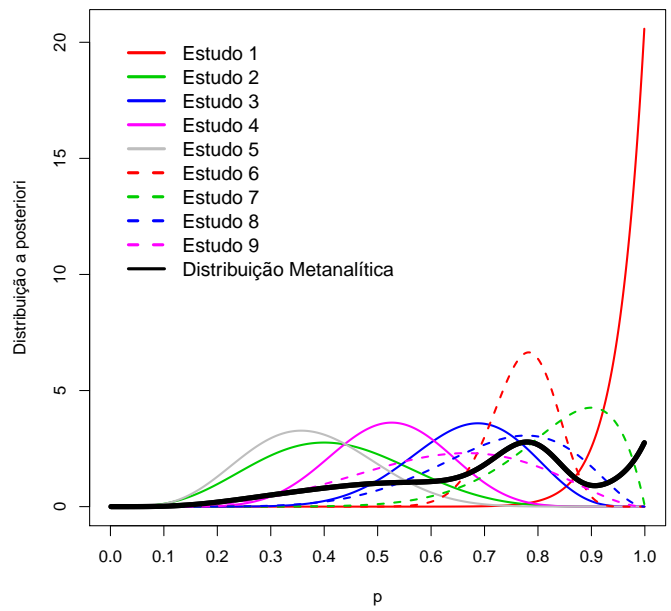

(b)

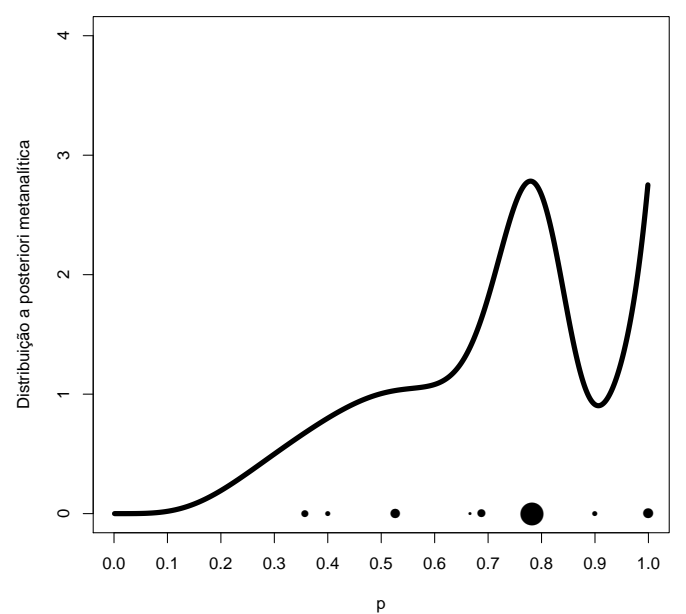

Figura 2.8: (a) Distribuições a posteriori de p para os nove estudos. (b) Distribuição a posteriori metanalítica de $p$.

As metodologias propostas na resolução desta metanálise diferem conceitualmente, o que implica a impossibilidade de comparação dos resultados obtidos, utilizando algum tipo de métrica para a escolha de um melhor modelo. Por exemplo, não faz sentido o uso da amplitude dos intervalos de credibilidade ou de confiança, como uma métrica de comparação, pois não necessariamente o melhor modelo, será aquele com menor amplitude. Não se está interessado na escolha de um melhor modelo, e sim na essência da metodologia proposta. Portanto, o que se discute aqui é a ideia e a interpretação das metodologias utilizadas.

Ao se observar apenas os dados da Tabela 2.1, nota-se que, provavelmente, existe um efeito no uso da droga SAMe e que este efeito, aparentemente, difere em alguns estudos. Há estudos com percentual de sucesso no uso da droga muito alto, como no Estudo 7 com $90 \%$ e no Estudo 1 com $100 \%$ de sucesso, e alguns estudos com baixo percentual de sucesso no uso da mesma droga, como o caso do Estudo 5 com apenas 36\%. Portanto, é nítido que a proporção de sucesso difira entre os estudos e a análise de heterogeneidade entre os estudos já não faça sentido.

É importante ressaltar que o método proposto vai além de observar a média ponderada dos estudos pertencentes à metanálise, como é o caso do método de DerSimonian e Laird. Neste trabalho, a metodologia proposta permite observar o comportamento do parâmetro de interesse através de sua distribuição a posteriori metanalítica. Também não há preocupação com a existência ou não da heterogeneidade entre os estudos, uma vez que esta já está incorporada neste modelo, diferentemente dos métodos usuais.

Na Figura 2.7, observa-se uma grande massa em torno de $75 \%$ que decai nas extremidades. Ou seja, o modelo hierárquico bayesiano acabou ignorando a informação de $100 \%$ de sucesso do Estudo 1 o que não ocorre com a metodologia proposta. A distribuição metanalítica de $p$ sugere a existência de três grupos, o primeiro, com percentual de sucesso em torno de $50 \%$, o segundo, com percentual em torno de $78 \%$ e o terceiro, com percentual de sucesso igual a $100 \%$. Assim, a metodologia proposta levou em conta toda a informação disponível e evidenciou, por exemplo, a influência do Estudo 1, fazendo com que o pesquisador reflita a seu respeito e sobre sua inclusão ou exclusão na metanálise.

\subsection{Aplicação}

\subsubsection{Exemplo 3: Metanálise para proporção}

Suponha estar interessado em verificar o sucesso na aplicação de uma nova técnica cirúrgica com 
relação à usual; que é aplicada em sete centros universitários de cinco estados brasileiros de três diferentes regiões. A Região Sudeste compreende a Universidade de São Paulo (USP), Universidade Federal de São Paulo (UNIFESP), Universidade Estadual de São Paulo (UNESP) e a Universidade Federal do Rio de Janeiro (UFRJ); a Região Sul, a Universidade Federal do Rio Grande do Sul (UFRGS) e o Instituto de Porto Alegre da Universidade Metodista do Sul (IPA) e a Região Nordeste, a Universidade de Pernambuco (UPE) e a Universidade Federal da Bahia (UFBA). Os resultados encontrados estão na Tabela 2.3. Este é um exemplo fictício, construído apenas para exemplificação da metodologia proposta.

Tabela 2.3: Prevalência do sucesso da nova técnica cirúrgica com relação à usual.

\begin{tabular}{cccccccc}
\hline & \multicolumn{7}{c}{ Centros } \\
\cline { 2 - 7 } & USP & UNIFESP & UNESP & UFRJ & UFRGS-IPA & UPE & UFBA \\
& $n_{1}=308$ & $n_{2}=33$ & $n_{3}=49$ & $n_{4}=17$ & $n_{5}=126$ & $n_{6}=73$ & $n_{7}=23$ \\
$Y_{1}(\%)$ & $Y_{2}(\%)$ & $Y_{3}(\%)$ & $Y_{4}(\%)$ & $Y_{5}(\%)$ & $Y_{6}(\%)$ & $Y_{7}(\%)$ \\
\hline Sucesso & $156(50,7)$ & $15(45,4)$ & $28(57,1)$ & $14(82,4)$ & $92(73,0)$ & $36(49,3)$ & $14(60,9)$ \\
\hline
\end{tabular}

Considere que a variável aleatória $X$ representa a aplicação da nova técnica cirúrgica, ou seja, $X=1$, se a cirurgia foi um sucesso com probabilidade $p$ ou $X=0$, se a cirurgia foi um fracasso com probabilidade $1-p$. Então, $X \mid p \sim \operatorname{Bernouli}(p)$ e sua distribuição de probabilidade é

$$
P(X=x \mid p)=p^{x}(1-p)^{1-x}
$$

Para o centro $j(j=1,2, \ldots, 7)$, suponha que $\mathbf{X}_{j}$ são variáveis aleatórias, condicionalmente independentes dado $p$. Considere $\boldsymbol{x}_{j}=\left(x_{1}, x_{2}, \ldots, x_{n_{j}}\right)^{T}$ uma amostra aleatória observada das variáveis $\mathbf{X}_{j}$. A função de verossimilhança para o $j$-ésimo centro é

$$
L_{j}\left(\boldsymbol{x}_{j}, p\right)=\prod_{i=1}^{n_{j}} p^{x_{i j}}(1-p)^{1-x_{i j}}=p^{y_{j}}(1-p)^{n_{j}-y_{j}}
$$

sendo $y_{j}=\sum_{i=1}^{n_{j}} x_{i j}$.

Considerando que a distribuição a priori de $p$ é Uniforme $(0,1)$ e a função de verossimilhança em (2.27), a distribuição a posteriori de $p$, para o $j$-ésimo centro, é

$$
\pi_{j}\left(p \mid \boldsymbol{y}_{j}\right)=\frac{\Gamma\left(n_{j}+2\right)}{\Gamma\left(y_{j}+1\right) \Gamma\left(n_{j}-y_{j}+1\right)} p^{y_{j}}(1-p)^{n_{j}-y_{j}} .
$$

Ou seja, $p \mid \boldsymbol{y}_{j} \sim \operatorname{Beta}\left(y_{j}+1, n_{j}-y_{j}+1\right), j=1, \ldots, 7$. Encontrada a distribuição a posteriori de $p$ para cada centro, o próximo passo será a obtenção da medida metanalítica.

Com base em (2.11) e considerando $\omega_{j}=\frac{n_{j}}{n} \operatorname{com} n=\sum_{j=1}^{7} n_{j}$, a distribuição a posteriori metanalítica de $p, \pi_{M}(p \mid \boldsymbol{y})$ é

$$
\pi_{M}(p \mid \boldsymbol{y})=\sum_{j=1}^{7}\left(\frac{n_{j}}{n}\right) \frac{\Gamma\left(n_{j}+2\right)}{\Gamma\left(y_{j}+1\right) \Gamma\left(n_{j}-y_{j}+1\right)} p^{y_{j}}(1-p)^{n_{j}-y_{j}} .
$$

Na Tabela 2.4 há algumas medidas resumo (média, moda, mediana, variância e intervalo HPD de $95 \%$ de credibilidade (HPD 95\%)) a posteriori de $p$ para cada centro. Na Figura 2.9, estão as distribuições a posteriori de $p$ para cada centro e sua distribuição a posteriori metanalítica. Note que o comportamento da distribuição a posteriori metanalítica de $p$ sugere a existência de dois grupos. O primeiro grupo, composto pela UFRJ e UFRGS-IPA, aparentemente, apresenta maior proporção de sucesso da nova técnica cirúrgica que o segundo grupo, composto pelos demais centros. Esta medida metanalítica permite ver as similaridades entre os centros, como uma análise de cluster. 
Em uma primeira análise, podem ser calculadas algumas medidas estatísticas e probabilidades por meio da distribuição a posteriori metanalítica de $p$ (Tabela 2.5).

Tabela 2.4: Sumário a posteriori de p para cada centro.

\begin{tabular}{cccccc}
\hline Centro & Média & Moda & Mediana & Variância & HPD 95\% \\
\hline USP & 0,51 & 0,51 & 0,51 & 0,01 & {$[0,45 ; 0,56]$} \\
UNIFESP & 0,46 & 0,45 & 0,46 & 0,06 & {$[0,30 ; 0,62]$} \\
UNESP & 0,57 & 0,57 & 0,57 & 0,02 & {$[0,43 ; 0,70]$} \\
UFRJ & 0,79 & 0,82 & 0,80 & 0,01 & {$[0,61 ; 0,95]$} \\
UFRGS-IPA & 0,73 & 0,73 & 0,73 & 0,01 & {$[0,65 ; 0,80]$} \\
UPE & 0,49 & 0,49 & 0,49 & 0,03 & {$[0,38 ; 0,60]$} \\
UFBA & 0,60 & 0,61 & 0,60 & 0,03 & {$[0,41 ; 0,78]$} \\
\hline
\end{tabular}

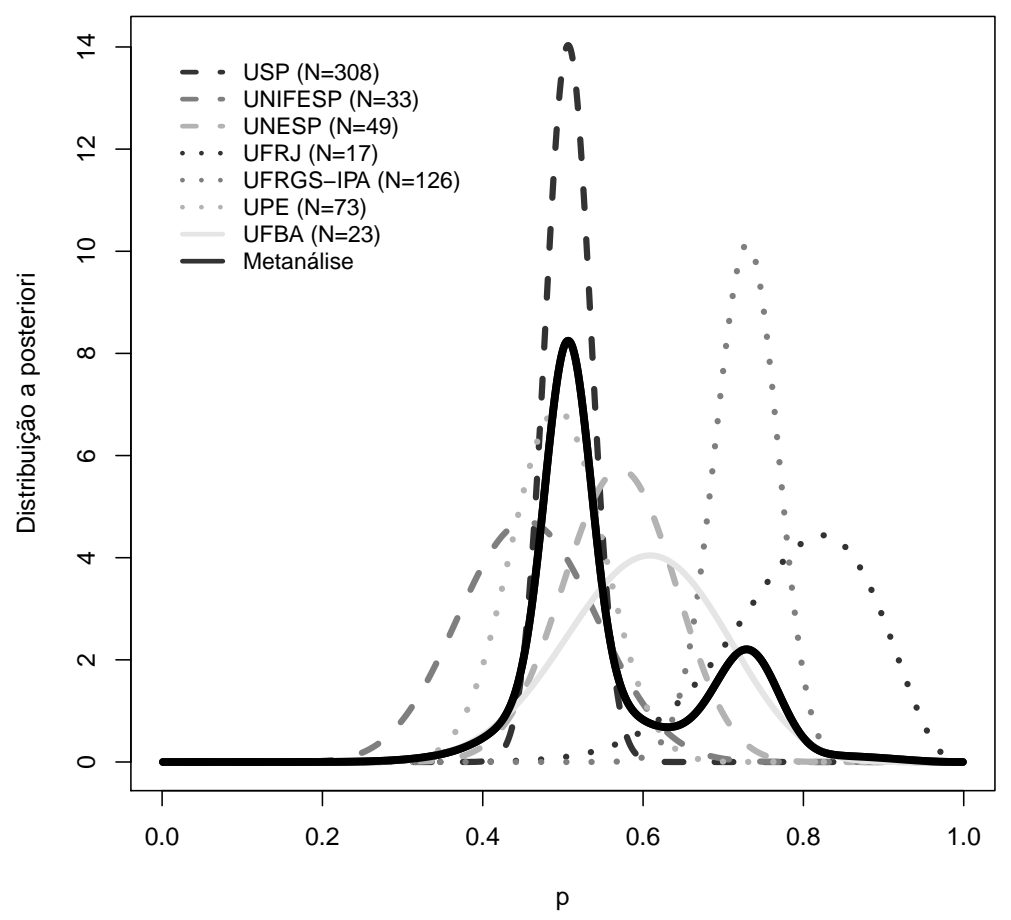

Figura 2.9: Distribuições a posteriori de p.

Tabela 2.5: Sumário da distribuição a posteriori metanalítica de p.

\begin{tabular}{cccccc}
\hline & Média & Mediana & Moda & Variância & HPD 95\% \\
\hline$p$ & 0,56 & 0,52 & $0,50(0,73)$ & 0,11 & {$[0,41 ; 0,79]$} \\
\hline
\end{tabular}

Ao se usar a média como medida-resumo da presente distribuição metanalítica de $p$, perdese informação. Isto porque há uma grande massa de dados maior que a média. Nesse caso, a distribuição a posteriori metanalítica de $p$ é uma distribuição bimodal, 0,73 é também um máximo local, portanto, é melhor utilizar a moda, como uma medida-resumo.

Também se pode calcular a distribuição preditiva, $p(z \mid \boldsymbol{y})$, de uma nova observação $z . z \mid p \sim$ $\operatorname{Bernoulli}(p)$ e sabe-se que $p(z \mid \boldsymbol{y})=\int_{0}^{1} f(z \mid p) \pi(p \mid \boldsymbol{y}) d p$ (Lee [2012]). Então, a função preditiva 
metanalítica é

$$
p(z \mid \boldsymbol{y})=\sum_{j=1}^{J} w_{j} \frac{B\left(z+y_{j}+1, n_{j}-y_{j}-z+2\right)}{B\left(y_{j}+1, n_{j}-y_{j}+1\right)} .
$$

Ao se calcular a distribuição preditiva para esses dados, tem-se que $P[Z=1 \mid \boldsymbol{y}]=0,56=1$ $P[Z=0 \mid \boldsymbol{y}]$. Esta probabilidade preditiva não é muito informativa, pois a chance de um novo paciente obter sucesso na nova técnica cirúrgica é de $56 \%$. Este resultado é como jogar uma moeda para predizer se o próximo paciente terá ou não sucesso na cirurgia. Portanto, os centros foram separados nos dois grupos descritos acima (Figura 2.9) e ambos foram analisados separadamente, conforme a mesma metodologia. É importante deixar claro que agora tem-se duas distribuições metanalíticas de $p, \pi_{M_{1}}\left(p_{1} \mid \boldsymbol{y}_{G_{1}}\right)$ e $\pi_{M_{2}}\left(p_{2} \mid \boldsymbol{y}_{G_{2}}\right)$, pois há dois grupos a serem examinados separadamente. A Figura 2.10(a) contém as distribuições a posteriori de $p_{1}$ para cada centro do Grupo 1 e sua respectiva distribuição a posteriori metanalítica. As distribuições a posteriori de $p_{2}$ para cada centro do Grupo 2 e a distribuição metanalítica de $p_{2}$ podem ser vistas na Figura 2.10(b). Apenas as distribuições metanalíticas estão na Figura 2.11.

(a) Grupo 1

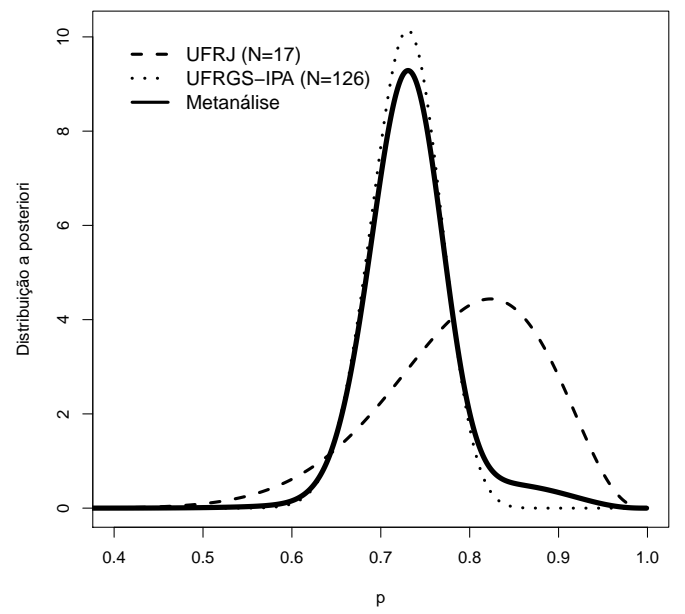

(b) Grupo 2

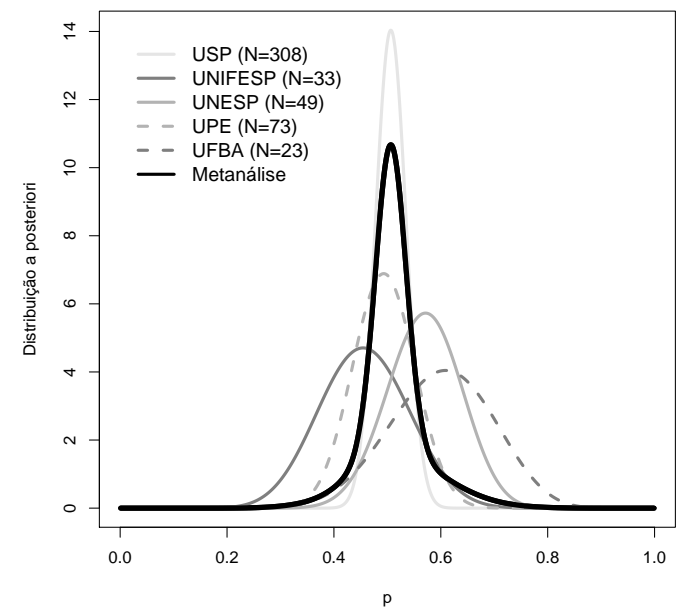

Figura 2.10: (a) Distribuiçôes a posteriori de $p_{1}$. (b) Distribuiçôes a posteriori de $p_{2}$.

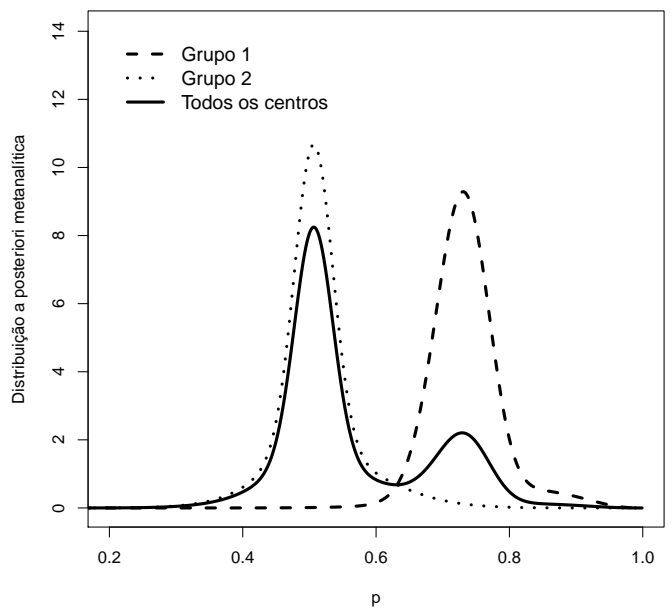

Figura 2.11: Distribuições a posteriori metanalíticas.

A medida metanalítica parece ser mais plausível, agora. Fica claro que havia alguma diferença 
entre os centros (Figura 2.10). Analisando separadamente os grupos, a média para o Grupo 1 é igual a 0,73 com intervalo HPD de $95 \%$ de credibilidade igual a $[0,64 ; 0,87]$ e para o Grupo $2,0,51$ e $[0,30 ; 0,66]$. Se forem calculadas as distribuições preditivas de ambos os grupos, será observado que $P\left[Z=1 \mid \boldsymbol{y}_{G_{1}}\right]=0,73$ e $P\left[Z=1 \mid \boldsymbol{y}_{G_{2}}\right]=0,51$. Este resultado é mais interessante que o anterior, pois, pode-se escolher realizar a nova cirurgia nos centros do Grupo 1 em lugar de se tratar em qualquer centro. Também pode-se calcular a probabilidade da proporção de sucessos na aplicação da nova técnica cirúrgica de $G_{1}$ ser maior que a proporção de sucessos de $G_{2}$, ou seja, $P\left[p_{1}>p_{2} \mid \boldsymbol{y}_{G_{1}}, \boldsymbol{y}_{G_{2}}\right]=0,73$. Suponha que se deseja testar a hipótese nula $H_{0}: p_{1}=p_{2}$ contra a hipótese alternativa $H_{a}: p_{1} \neq p_{2}$. Com o uso do teste FBST (Full Bayesian Significance Test) para testar esta hipótese (Pereira e Stern [1999]), o valor de evidência contra $H_{0}$ é $\overline{e v}=0,94$. Ou seja, é uma alta evidência contra a hipótese nula, indicando que a proporção de sucessos na aplicação da técnica cirúrgica é diferente nos dois grupos.

\subsubsection{Exemplo 4: Metanálise para diferença entre médias}

Considere a metanálise sobre os efeitos do bloqueio duplo do sistema renina-angiotensina na doença diabética renal (DDR), apresentada por Pham et al. [2012]. A proposta desta metanálise será determinar os benefícios relativos (redução da proteinúria e da pressão arterial sistólica (PAS)) e riscos (hipercalemia), assim como os efeitos na taxa de filtração glomerular (TFG) da terapia combinada versus monoterapia em pacientes com DDR. Para exemplificar a metodologia proposta, consideram-se os dados da Figura 4b deste artigo (Tabela 2.6), que se referem à diferença na PAS, depois da combinação do tratamento com ALDOA e terapia (grupo experimental) versus monoterapia (grupo controle). O objetivo desta metanálise é comparar os grupos com respeito à PAS.

Tabela 2.6: Medidas resumo da PAS.

\begin{tabular}{ccccccc}
\hline & \multicolumn{3}{c}{ Experimental } & \multicolumn{3}{c}{ Controle } \\
\cline { 2 - 7 } Estudo & $\bar{x}_{1 j}$ & $s_{1 j}$ & $n_{1 j}$ & $\bar{x}_{2 j}$ & $s_{2 j}$ & $n_{2 j}$ \\
\hline Rossing 2005 & 132 & 13 & 20 & 138 & 13 & 20 \\
Schjoedt 2005 & 136 & 18 & 20 & 144 & 13 & 20 \\
Schjoedt 2006 & 142 & 18 & 20 & 146 & 18 & 20 \\
\hline
\end{tabular}

A variável aleatória $X_{i j k}$ representa a pressão sistólica sanguínea do $i$-ésimo indivíduo do $j$ ésimo estudo $(j=1, \ldots, 5)$ e $k$-ésimo grupo $(k=1,2)$. Considere que $X_{i j k} \mid \mu_{k}, \sigma_{k}^{2} \sim \operatorname{Normal}\left(\mu_{k}, \sigma_{k}^{2}\right)$ e o problema é similar ao Exemplo 1. Portanto, a função de verossimilhança para o $j$-ésimo estudo do $k$-ésimo grupo é

$$
L_{j k}\left(\boldsymbol{x}_{j k}, \mu_{k}, \sigma_{k}^{2}\right) \propto\left(\sigma_{k}^{2}\right)^{-n_{j k} / 2} \exp \left\{-\frac{1}{2 \sigma_{k}^{2}}\left[\left(n_{j k}-1\right) s_{j k}^{2}+n_{j k}\left(\mu-\bar{x}_{j k}\right)^{2}\right]\right\} .
$$

Considerando a priori de Jeffreys, $\pi_{k}\left(\mu_{k}, \sigma_{k}^{2}\right) \propto\left(\sigma_{k}^{2}\right)^{-1}$, para ambos os estudos do $k$-ésimo grupo e a função de verossimilhança em (2.31), a distribuição a posteriori de $\mu_{k}$ e $\sigma_{k}^{2}$ é Normal-GamaInversa, isto é,

$$
\left(\mu_{k}, \sigma_{k}^{2} \mid \boldsymbol{x}_{j k}\right) \sim N G I\left(\bar{x}_{j k}, n_{j k}, \frac{n_{j k}-1}{2}, \frac{\left(n_{j k}-1\right) s_{j k}^{2}}{2}\right) .
$$

Portanto, baseando-se em (2.11) e considerando $\omega_{j k}=\frac{n_{j k}}{\sum_{j=1}^{3} n_{j k}}$, a distribuição a posteriori metanalítica de $\left(\mu_{k}, \sigma_{k}^{2}\right)$ é

$$
\pi_{M_{k}}\left(\mu_{k}, \sigma_{k}^{2} \mid \boldsymbol{x}_{j k}\right)=\pi_{k}\left(\mu_{k}, \sigma_{k}^{2}\right) \sum_{j=1}^{3} w_{j k} L_{j k}\left(\mu_{k}, \sigma_{k}^{2} \mid \boldsymbol{x}_{j k}\right) .
$$


Lembrando que a distribuição a posteriori conjunta de $\mu_{k}$ e $\sigma_{k}^{2}$ depende dos dados apenas por meio das estatísticas suficientes $\left(n_{j k}, \bar{x}_{j k}, s_{j k}^{2}\right)$; e, neste caso, os resultados obtidos pela metanálise baseada na literatura são os mesmos da metanálise padrão ouro.

Na Figura 2.12(a), estão as curvas de nível das distribuições a posteriori de $\left(\mu_{1}, \sigma_{1}^{2}\right)$ e na Figura 2.12(b), estão as curvas de nível das distribuições a posteriori de $\left(\mu_{2}, \sigma_{2}^{2}\right)$. As curvas de nível da distribuição a posteriori metanalítica de $\left(\mu_{1}, \sigma_{1}^{2}\right)$ podem ser vistas na Figura 2.12(c) e as curvas de nível da distribuição a posteriori metanalítica de $\left(\mu_{2}, \sigma_{2}^{2}\right)$, na Figura 2.12(d). As regiões HPD com $95 \%$ de credibilidade para os parâmetros de interesse encontram-se na Figura 2.13. Por fim, na Figura 2.14 estão os gráficos tridimensionais da distribuição a posteriori metanalítica de $\left(\mu_{1}, \sigma_{1}^{2}\right)$ e de $\left(\mu_{2}, \sigma_{2}^{2}\right)$. Ao observar as Figuras 2.13 e 2.14 pode-se notar que as distribuições dos grupos aparentemente são próximas. Entretanto, seria interessante testar se as distribuições metanalíticas de $\left(\mu_{1}, \sigma_{1}^{2}\right)$ e $\left(\mu_{2}, \sigma_{2}^{2}\right)$ são iguais. Utilizando o FBST para testar a hipótese nula $H_{0}: \mu_{1}=\mu_{2} ; \sigma_{1}^{2}=$ $\sigma_{2}^{2}$, obtém-se um valor de evidência a favor da hipótese nula igual a $0,97, e v=0,97$. Ou seja, há uma grande evidência de que as distribuições metanalíticas dos grupos são similares.
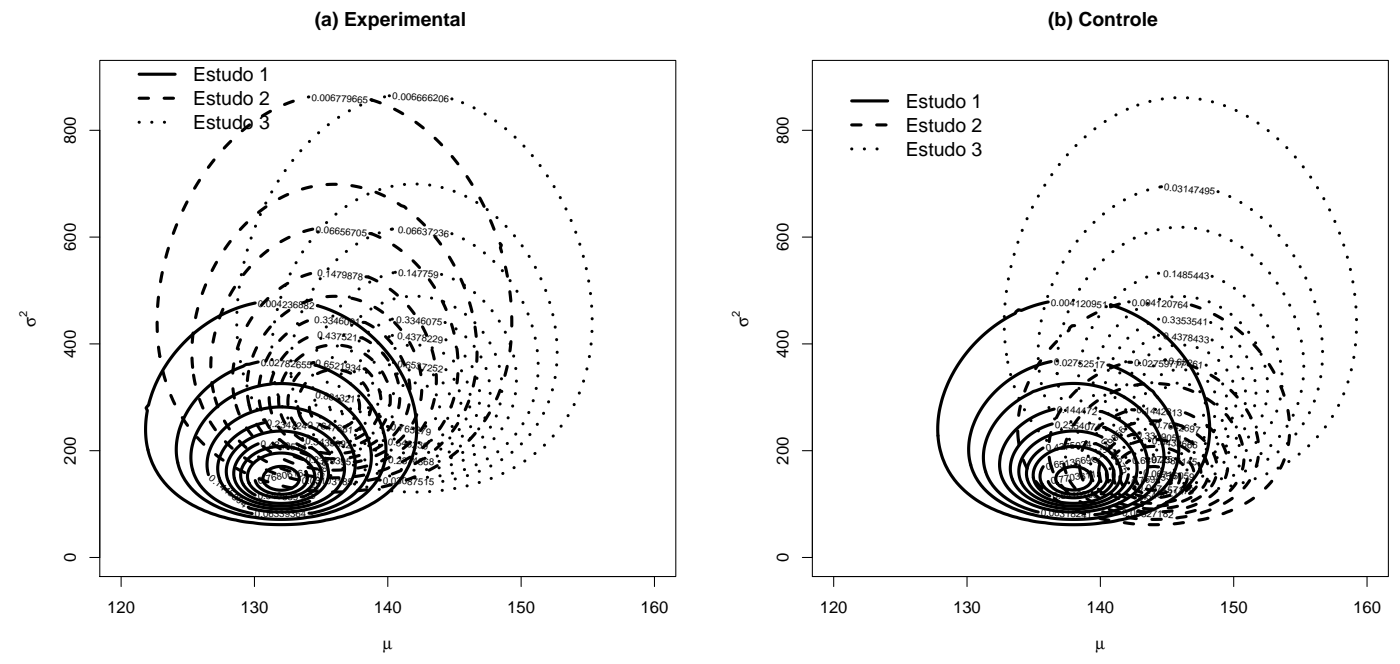

(c) Metanálise - Experimental
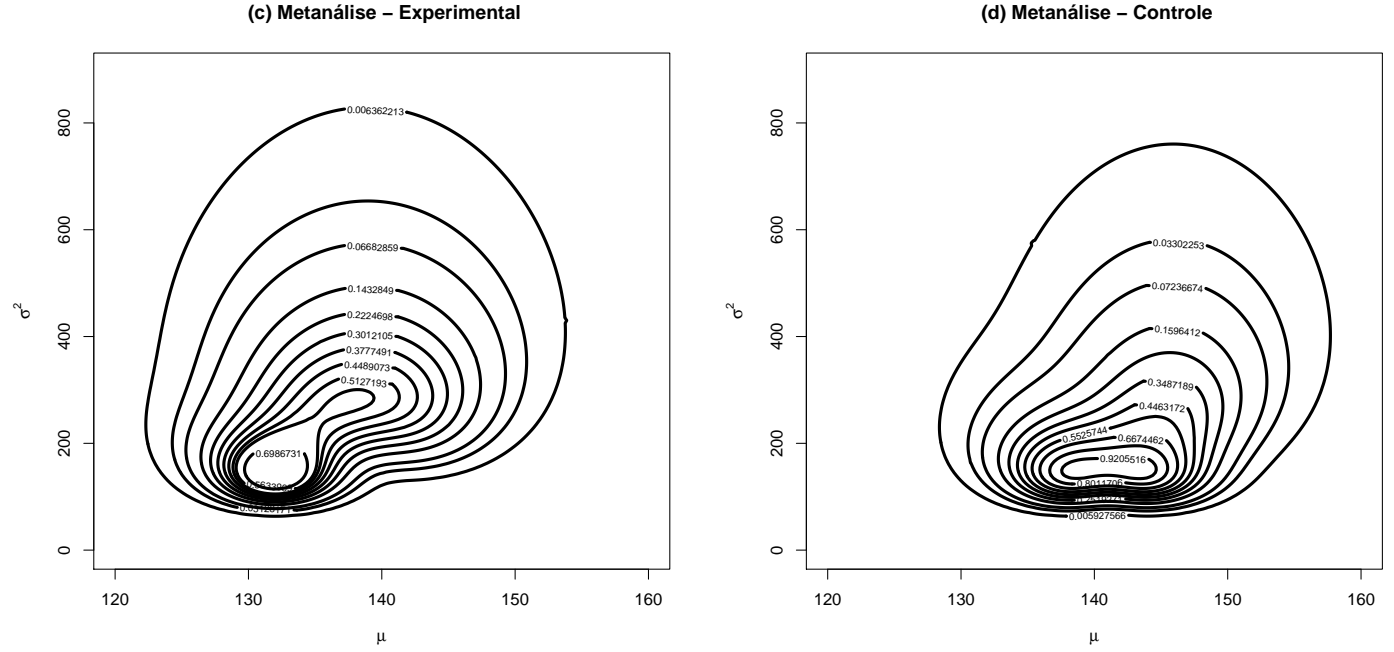

Figura 2.12: Curvas de nivel. 
(a) Experimental

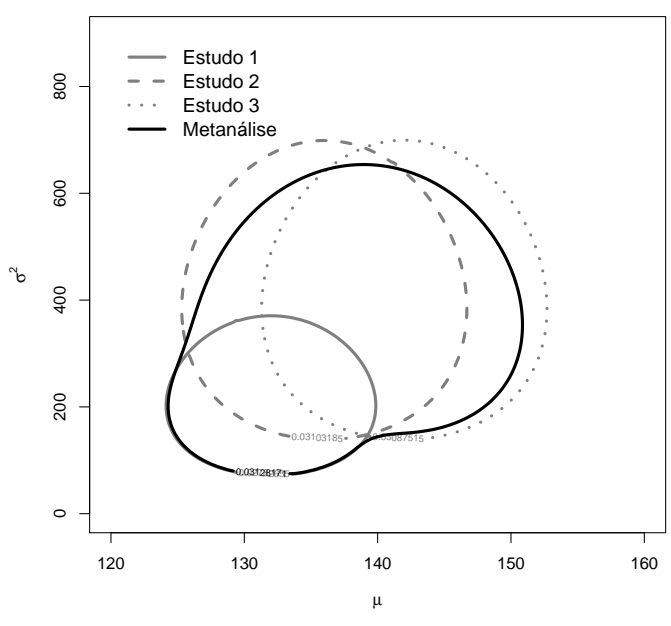

(c) Metanálise - HPD

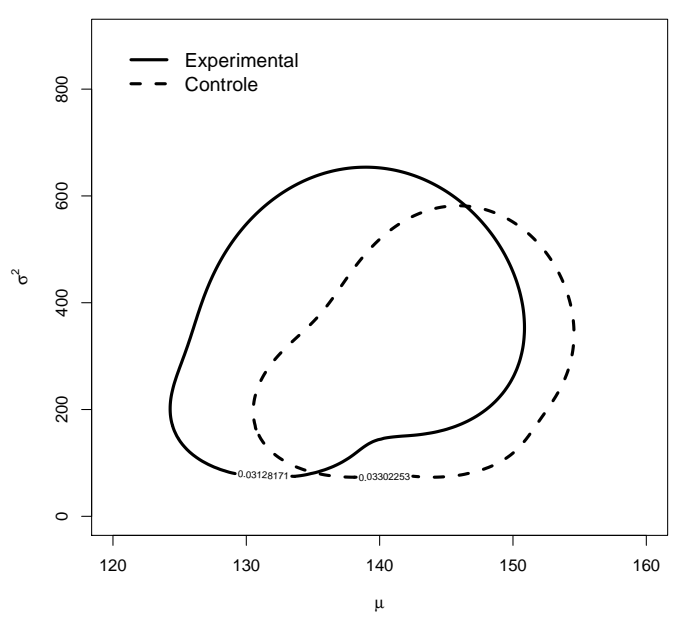

(b) Controle

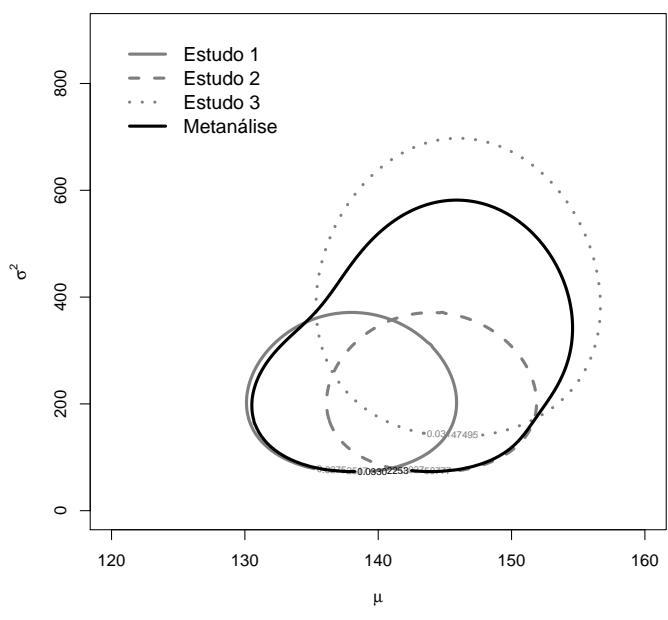

(d) Metanálise

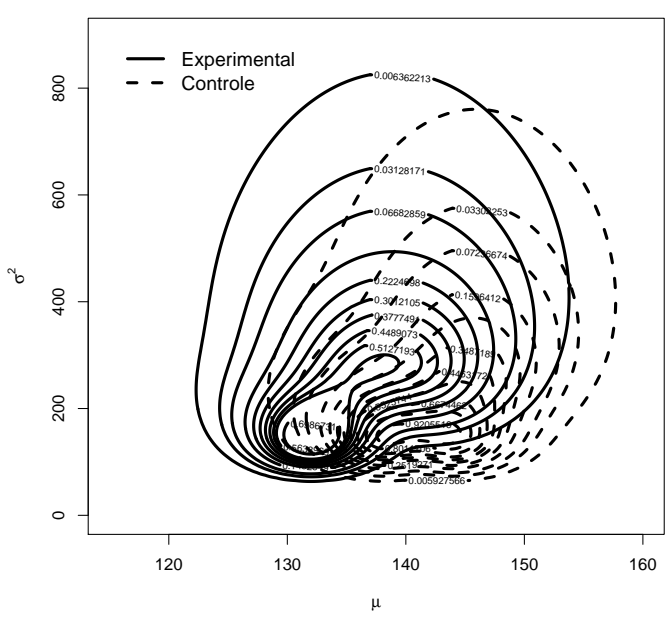

Figura 2.13: (a) Região HPD de 95\% de credibilidade para o grupo experimental. (b) Região HPD de $95 \%$ de credibilidade para o grupo controle. (c) Região HPD de $95 \%$ de credibilidade apenas para os parâmetros metanalíticos. (d) Curvas de nível das distribuições a posteriori metanalíticas de ambos os grupos.
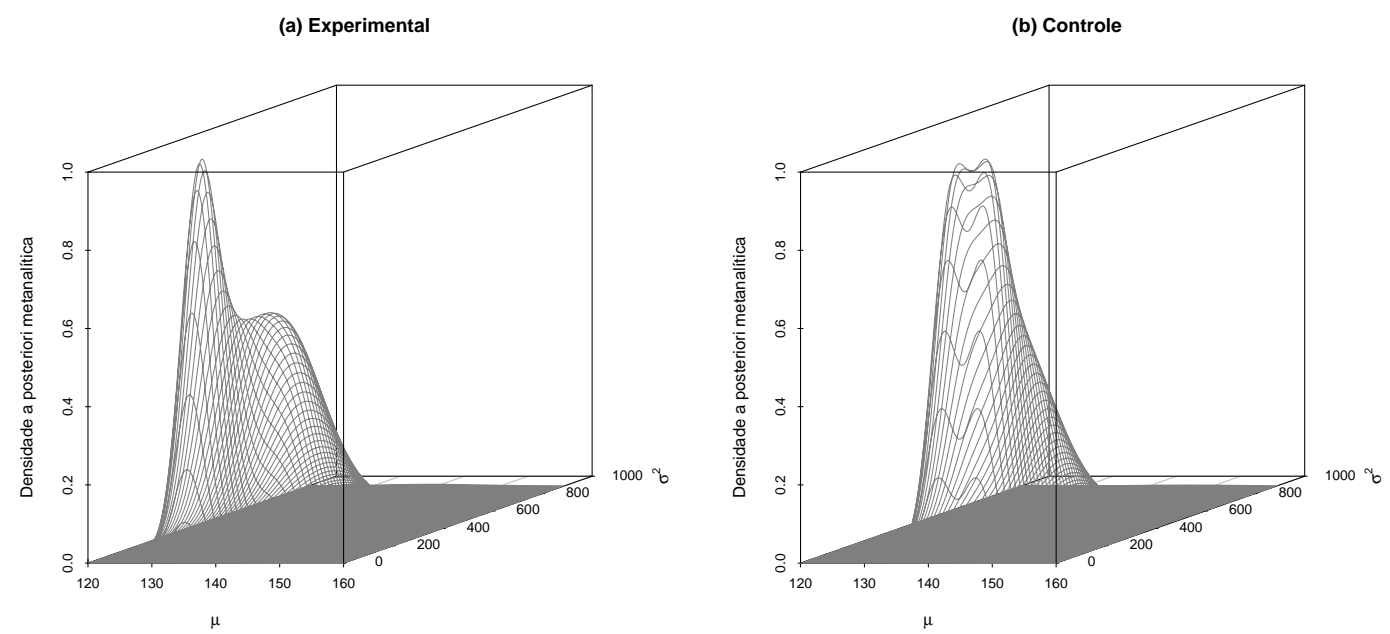

Figura 2.14: Distribuição a posteriori metanalítica de $\mu$ e $\sigma^{2}$ para o grupo experimental (a) e para o grupo controle (b). 
Outro modo de se trabalhar com estes dados é considerar a diferença $Y_{j}=X_{1 j}-X_{2 j}$. Logo, $Y_{j} \mid \delta, \tau \sim N(\delta, \tau)$, onde $\delta=\mu_{1}-\mu_{2}$ e $\tau=\sigma_{1}^{2}+\sigma_{2}^{2}$, então, pode-se encontrar a medida metanalítica dada em (2.11) de forma similar à anterior. A função de verossimilhança para o $j$-ésimo estudo é

$$
L_{j}\left(\boldsymbol{y}_{j}, \delta, \tau\right) \propto(\tau)^{-n_{j} / 2} \exp \left\{-\frac{1}{2 \tau}\left[\left(n_{j}-1\right) s_{p_{j}}^{2}+n_{j}\left(\delta-\bar{y}_{j}\right)^{2}\right]\right\}
$$

sendo $s_{p_{j}}^{2}=s_{1 j}^{2}+s_{2 j}^{2}$ e $\bar{y}_{j}=\bar{x}_{1 j}-\bar{x}_{2 j}$.

Considerando a priori de Jeffreys $\pi(\delta, \tau) \propto 1 / \tau$ e a função de verossimilhança em (2.34), a distribuição a posteriori conjunta de $\delta$ e $\tau$ segue distribuição Normal-Gama-Inversa. Ou seja,

$$
\left(\delta, \tau \mid \boldsymbol{y}_{j}\right) \sim N G I\left(\bar{y}_{j}, n_{j}, \frac{n_{j}-1}{2}, \frac{\left(n_{j}-1\right) s_{p_{j}}^{2}}{2}\right)
$$

Portanto, novamente, baseado em (2.11) e considerando $\omega_{j}=\frac{n_{j}}{\sum_{j=1}^{3} n_{j}}$, a distribuição a posteriori metanalítica de $\delta$ e $\tau, \pi_{M}(\delta, \tau \mid y)$ é

$$
\pi_{M}(\delta, \tau \mid \boldsymbol{y})=\pi(\delta, \tau) \sum_{j=1}^{3} w_{j} L_{j}\left(\delta, \tau \mid \boldsymbol{y}_{j}\right)
$$

Na Figura 2.15(a), estão as curvas de nível das distribuições conjuntas a posteriori de $\delta$ e $\tau$ para cada estudo. Na Figura 2.15(b), está a distribuição conjunta a posteriori metanalítica de $(\delta, \tau)$ e na Figura 2.15(c), está o gráfico tridimensional de $\pi_{M}(\delta, \tau \mid \boldsymbol{y})$. A estimação por região HPD de 95\% de credibilidade está na Figura 2.15(d). Observando a Figura 2.15 pode-se notar que a média de $\pi_{M}(\delta, \tau \mid \boldsymbol{y})$ está, aparentemente, distante de zero, indicando um suposto efeito do tratamento. Para se testar a eficácia do tratamento, testa-se via FBST se a média da distribuição conjunta a posteriori metanalítica de $(\delta, \tau)$ é igual a zero, ou seja, a hipótese nula é $H_{0}: \delta=0$. O valor de evidência contra a hipótese nula encontrado foi $\overline{e v}=0,44$, indicando que não há evidência suficiente contra $H_{0}$, entretanto, $P[\delta<0]=0,86$. A distribuição a posteriori marginal de $\delta$ para cada estudo e sua distribuição a posteriori metanalítica podem ser vistas na Figura 2.16. O valor esperado a posteriori de $\delta$ é $E[\delta \mid \boldsymbol{y}]=-6$ e o intervalo HPD de $95 \%$ de credibilidade é $[-17,4 ; 5,6]$. A medida metanalítica encontrada em Pham et al. [2012], calculada pelo método de DerSimonian e Laird, foi igual a $-6.2 \mathrm{mmHg}$ com intervalo de confiança de $95 \%$ igual a $[-11,57 ;-0,73]$. Este resultado mostrou significância a favor do grupo experimental, divergindo dos resultados encontrados pelo método bayesiano proposto em razão da incorporação da variabilidade neste método, o que o torna mais confiável que os procedimentos usuais. 

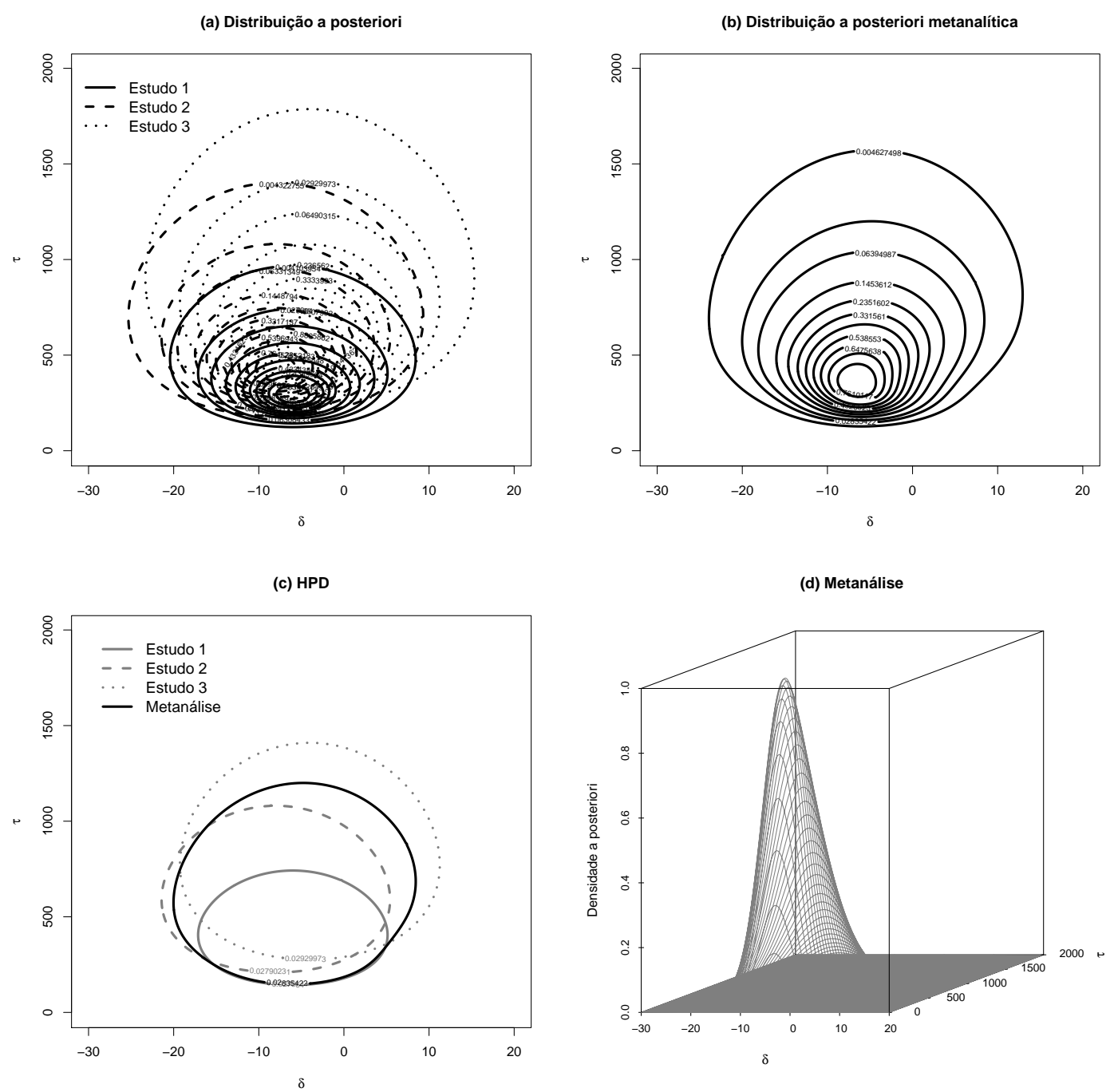

Figura 2.15: (a) Curvas de nivel das distribuições a posteriori de $(\delta, \tau)$ para cada estudo. (b) Curvas de nível da distribuição a posteriori metanalítica de $(\delta, \tau)$. (c) Distribuição a posteriori metanalítica de $(\delta, \tau)$ em 3D. (d) Região HPD de $95 \%$ de credibilidade.

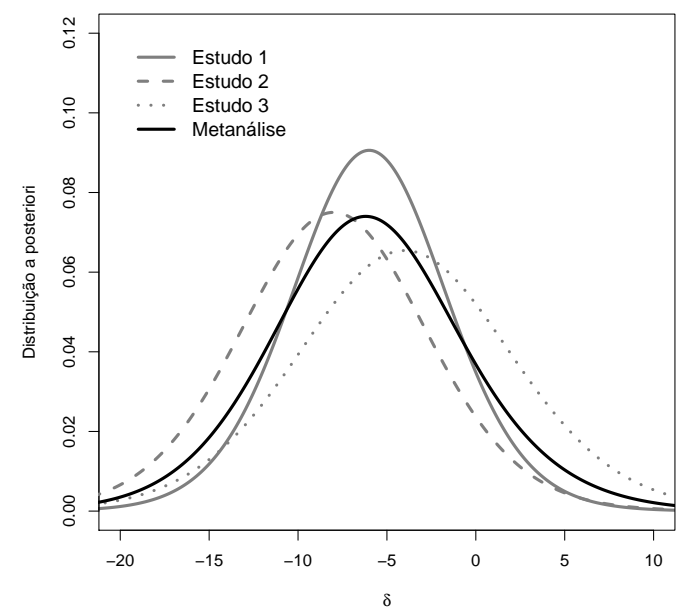

Figura 2.16: Distribuições a posteriori marginal de $\delta$. 


\subsection{Discussão}

O método usualmente utilizado para a realização de uma metanálise baseada na literatura, apoia-se no modelo de DerSimonian e Laird. Este método incorpora a variabilidade existente entre os estudos na ponderação das medidas de efeito de cada estudo para, então, produzir uma medida metanalítica. Tal método tende a dar maior peso aos estudos pequenos, desde que estes estudos possam refletir um viés de publicação, o uso desse modelo pode enfatizar evidências pobres à custa de uma boa evidência (Petitti [2000]). Além do mais, observou-se que a medida metanalítica resultante é pobre, pode ser viesada e não fornece muita informação sobre o parâmetro em questão. O modelo de mistura finita utilizado em metanálise apresentou dificuldade na interpretação dos resultados e, além disso, não levou em conta a variabilidade existente entre os estudos. O modelo hierárquico também deixou a desejar na utilização de toda a informação disponível, ignorando pontos importantes do problema apresentado. Entretanto, tais problemas não ocorreram com a metanálise bayesiana proposta.

A medida metanalítica proposta é uma mistura de distribuições a posteriori do parâmetro de interesse, $\theta$, de cada estudo. É importante ressaltar que esse método produz como medida metanalítica uma distribuição de probabilidade, denominada de distribuição a posteriori metanalítica de $\theta$. Por meio desta distribuição, podem ser calculadas a esperança e a variância a posteriori de $\theta$, além de outras medidas. Portanto, a metodologia proposta é muito mais informativa, intuitiva e precisa que as existentes. No Capítulo 3, a metodologia proposta é aplicada em dados de análise de sobrevivência. 


\title{
Capítulo 3
}

\section{Metanálise para dados de sobrevivência}

\begin{abstract}
Resumo: Neste capítulo, aplica-se a metodologia proposta no Capitulo 2 em dados de sobrevivência. Na análise de sobrevivência, a variável resposta é sempre o tempo até a ocorrência de um evento, denominado tempo de sobrevivência. Uma característica importante desse tipo de dados é a presença de censura, assim, neste trabalho aborda-se o mecanismo de censura mais geral, a censura intervalar. A ideia principal consiste em utilizar, sob uma perspectiva bayesiana, o modelo Weibull, em razão de sua flexibilidade, para estimar as curvas de sobrevivência de cada estudo pertencente à metanálise e obter uma curva de sobrevivência metanalítica. Esta curva metanalítica é encontrada, utilizando a distribuição a posteriori metanalítica dos parâmetros do modelo em questão. Alguns exemplos com dados simulados e reais também são apresentados e discutidos.
\end{abstract}

Palavras-chave: Análise de sobrevivência, Dados censurados, Metanálise

\subsection{Introdução}

Nas últimas décadas, a análise de sobrevivência vem sendo bastante desenvolvida em razão do aprimoramento de técnicas estatísticas combinadas com o avanço computacional. A análise estatística dos dados relacionados ao tempo vem ganhando importância em muitas áreas, incluindo a biomédica, engenharia e ciências sociais, aumentando a produção científica e a disponibilização das informações.

Como referido no Capítulo 2, o modo usual de se fazer metanálise é considerar apenas as medidas de efeito de cada estudo e, então, combiná-las utilizando a média ponderada desses efeitos. Embora se saiba sobre a impossibilidade da realização desse tipo de metanálise com dados de sobrevivência, muitos estudos fazem uma adaptação dos dados para trabalharem desse modo, por causa da complexidade da realização da metanálise caso a caso (Egger et al. [2009]). Neste caso, a variável tempo de sobrevivência é transformada em uma variável dicotômica ( 0 , se o paciente morreu ou 1 se o paciente estiver vivo), calcula-se como medida de efeito o risco em um determinado tempo e, então, realiza-se a metanálise do modo usual. Note que, ao se fazer isso, muita informação é perdida e o foco do problema é alterado pois, não se trabalha mais com tempo de vida dos pacientes, mas, sim, com a proporção de pacientes vivos. Portanto, neste trabalho é dada ênfase à metanálise caso a caso para dados de sobrevivência, sob a abordagem bayesiana, apresentada no Capítulo 2.

$\mathrm{Na}$ análise de sobrevivência, a variável resposta é sempre o tempo até a ocorrência de um evento de interesse. Uma possível característica dos dados de sobrevivência é a presença de censura, que se resume à observação incompleta da variável tempo, causada por algum fator externo. Existem vários tipos de censura, como à direita, à esquerda e intervalar. Por exemplo, considere um estudo clínico em que o acontecimento de interesse seja a morte de um indivíduo, após o diagnóstico de um determinado tumor maligno; se o indivíduo estiver vivo no final do estudo, tem-se uma observação censurada à direita. A censura à esquerda ocorre quando o tempo de vida é menor que o tempo 
observado. Este tipo de censura é menos frequente que à direita. Considere um estudo em que o acontecimento de interesse é o desenvolvimento de um determinado tumor; os indivíduos que já possuem metástases, quando são observados, dão origem a observações censuradas à esquerda. Já a censura intervalar, ocorre quando não se conhece o instante exato de morte de um indivíduo, mas sabe-se que esta verificou-se dentro de um determinado intervalo de tempo. Portanto, neste trabalho, o mecanismo de censura intervalar é abordado, visto que possui como casos especiais os mecanismos de censura à esquerda e à direita, mais comum em estudos médicos.

A ideia principal deste trabalho foi utilizar o modelo Weibull, pela sua flexibilidade, para estimar as curvas de sobrevivência de cada estudo e então, obter uma curva de sobrevivência metanalítica. Esta curva é encontrada via distribuição a posteriori metanalítica dos parâmetros do modelo em questão, assim como em (2.11).

A Seção 2 contém uma breve revisão sobre a análise de sobrevivência. A Seção 3 apresenta a metanálise proposta no Capítulo 2 para dados de sobrevivência. Na Seção 4, são apresentadas diversas aplicações de metanálise para dados de sobrevivência. O primeiro exemplo, para o tempo de vida, considera o modelo Exponencial para dados completos e censurados e o segundo exemplo, o modelo Log-normal. Estes dois exemplos são casos especiais de metanálise caso a caso iguais a metanálise baseada na literatura, o que raramente acontece com dados de sobrevivência. Apresenta-se também, um exemplo de metanálise com modelo de riscos competitivos e um exemplo de metanálise com modelo de regressão em análise de sobrevivência. Finalmente, na Seção 5, encontram-se algumas considerações.

\subsection{Análise de sobrevivência}

A teoria para dados de sobrevivência tem como objetivo o acompanhamento de unidades observacionais até a ocorrência de algum evento de interesse. Em análise de sobrevivência, a variável resposta é sempre o tempo até a ocorrência desse evento: morte de um paciente em tratamento, cura de um doente em tratamento, falha de um equipamento, etc. O tempo entre o início da observação e a ocorrência do evento de interesse recebe diferentes nomes, de acordo com a área de interesse do estudo: tempo de sobrevivência, tempo de vida, tempo de falha, etc. Os dados de sobrevivência podem reunir não só os tempos de vida, mas também um conjunto de variáveis observáveis que pode ou não estar relacionado com esses tempos, tais como as características do próprio indivíduo (gênero, idade, etc.) e/ou fatores externos ao indivíduo (tratamento a que foi sujeito, fatores ambientais, etc.). Estas variáveis são conhecidas como covariáveis ou variáveis explicativas.

Como dito anteriormente, uma possível característica dos dados de sobrevivência é a presença de censura, esta ocorre, quando não é possível observar o acontecimento de interesse durante o período de tempo em que o indivíduo está em observação. Vale ressaltar que, mesmo censurados, todos os resultados procedentes de um estudo de sobrevivência devem ser utilizados na análise. Pois, mesmo sendo incompletas, as observações censuradas fornecem informações sobre o tempo de vida de indivíduos e a omissão das censuras no cálculo das estatísticas de interesse pode levar à conclusões viciadas (Colosimo e Giolo [2006]).

O principal tipo de censura discutido neste trabalho é o intervalar, que tem como casos especiais o tempo exato de falha, os dados censurados à direita e à esquerda. No caso de censura intervalar, os tempo de vida $T$ não são exatamente conhecidos. Sabe-se apenas que o evento de interesse, para os indivíduos observados, ocorreu em algum tempo dentro do intervalo $(U, V]$, com $U<T \leq V$. Esta situação acontece, por exemplo, quando a ocorrência do evento de interesse é monitorada em visitas médicas de rotina. Se o evento ocorrer exatamente em uma das visitas, o tempo exato de falha será observado, ou seja, $T=U=V$. Além disso, nos dados censurados à direita, sabe-se que o evento de interesse não ocorreu antes da última visita e que, provavelmente, ocorrerá em um momento futuro. Ou seja, $T$, provavelmente, ocorrerá em $(U, \infty)$, onde $U$ é o tempo decorrido, desde o início do estudo até à última visita e $V=\infty$. Similarmente, sabe-se que, para os indivíduos censurados à esquerda, o evento de interesse ocorreu antes da primeira visita. Ou seja, $T$ verificou-se em $(0, V]$, 
com $U=0$, representando o início do estudo e $V$, o tempo decorrido desde o início do estudo até a primeira visita (Kleinbaum e Klein [2005]).

O comportamento da variável aleatória contínua e não negativa, tempo de sobrevivência, $T \geq 0$, pode ser representada pela função densidade $(f(t))$, função de sobrevivência $(S(t))$ e função de risco $(h(t))$. Estas três funções podem ser utilizadas para descrever diferentes aspectos fornecidos pelo conjunto de dados do tempo de sobrevivência (Lawless [2003]).

A função densidade de probabilidade é definida, como o limite da probabilidade de um indivíduo falhar (morrer) no intervalo de tempo $[t, t+\Delta t]$ por unidade de tempo, e é expressa por

$$
f(t)=\lim _{\Delta t \rightarrow 0^{+}} \frac{P(t \leq T<t+\Delta t)}{\Delta t},
$$

em que $f(t) \geq 0$, para todo $x$.

A função de sobrevivência $S(x)$ ou confiabilidade $R(x)$ é definida, como a probabilidade de um indivíduo sobreviver mais que um determinado tempo $t$, ou seja, não falhar após um certo tempo, e é expressa por

$$
S(t)=P(T>t)=1-F(t)
$$

no qual $F(t)=\int_{0}^{t} f(u) d u$, representa a função de distribuição acumulada. $S(t)$ é uma função monótona decrescente com $S(0)=1$ e $S(\infty)=\lim _{t \rightarrow \infty} S(t)=0$.

Pela curva de sobrevivência, pode-se comparar, por exemplo, dois ou mais fatores de risco de um indivíduo. Assim, uma curva de sobrevivência com declive acentuado representa curto tempo de vida, e uma curva com aclive, longo tempo de vida. Já a função de risco analisa a taxa de falha de um indivíduo e descreve como a probabilidade de falha modifica-se com o passar do tempo.

A função de risco $h(t)$ também conhecida como taxa de falha, é definida como o limite da probabilidade de um indivíduo falhar no intervalo de tempo $[t, t+\Delta t]$, dado que o mesmo tenha sobrevivido até o tempo $t$, e é expressa por

$$
h(t)=\lim _{\Delta t \rightarrow 0^{+}} \frac{P(t \leq T<t+\Delta t \mid T \geq t)}{\Delta t}=\frac{f(t)}{S(t)} .
$$

Esta função analisa a probabilidade de falha (risco) de um determinado indivíduo e descreve como a probabilidade instantânea de falha modifica-se com o passar do tempo. Pode apresentar diferentes comportamentos, como: crescente, decrescente, constante ou combinada. A função de risco é mais importante que a de sobrevivência, pois podem ser encontradas funções de sobrevivência parecidas e, no entanto, diferentes funções de risco. Será dado ênfase ao modelo Weibull em razão de sua flexibilidade e por acomodar diferentes formas da função de risco.

\subsubsection{Construção da função de verossimilhança para dados censurados}

$\mathrm{Na}$ construção da função de verossimilhança, a natureza intervalar dos dados deve ser levada em consideração. Então, cada indivíduo contribui com uma informação específica na função de verossimilhança (Klein e Moeschberger [1997]). Por exemplo, se o $i$-ésimo indivíduo apresenta tempo de falha exato, sua contribuição na função de verossimilhança será a probabilidade da ocorrência do evento de interesse nesse tempo. Esta contribuição é dada por meio da função densidade de $T$ nesse respectivo tempo. Se o $i$-ésimo indivíduo for censurado à direita, sua contribuição será a função de sobrevivência de $T$ avaliada no último tempo da visita. Similarmente, se o $i$-ésimo indivíduo for censurado à esquerda, sua contribuição será a função distribuição acumulada de $T$ avaliada no primeiro tempo da visita. Finalmente, se o $i$-ésimo indivíduo apresentar tempo de falha em um certo intervalo de tempo, sua contribuição será dada pela probabilidade do tempo de ocorrência do evento pertencer a esse intervalo (Colosimo e Giolo [2006]). A função de verossimilhança para 
dados com censura intervar é dada por:

$$
L(\boldsymbol{t}, \theta)=\prod_{i \in F} f\left(t_{i}, \theta\right) \prod_{i \in C D} S\left(t_{i}, \theta\right) \prod_{i \in C E}\left[1-S\left(t_{i}, \theta\right)\right] \prod_{i \in C I}\left[S\left(u_{i}, \theta\right)-S\left(v_{i}, \theta\right)\right],
$$

onde $F$ é o conjunto, contendo os índices cujas observações falharam, $C D$ é o conjunto contendo os índices cujas observações são censurada à direita, $C E$ é o conjunto de índices em que as observações são censuradas à esquerda e $C I$ é o conjunto contendo os índices, cujas observações são censuras intervalares. Note que $\left[S\left(u_{i}, \theta\right)-S\left(v_{i}, \theta\right)\right]$ é a probabilidade do tempo real de falha $T_{i}$ estar em $\left(u_{i}, v_{i}\right]$.

Simplificando (3.4), pode-se construir a função de verossimilhança para dados de natureza intervalar, $\left(U_{i}, V_{i}\right]$, da seguinte forma

$$
L(\boldsymbol{t}, \theta)=\prod_{i=1}^{n} f\left(t_{i}, \theta\right)^{\delta_{i}}\left[F\left(v_{i}, \theta\right)-F\left(u_{i}, \theta\right)\right]^{1-\delta_{i}},
$$

onde $\delta_{i}$ é uma variável aleatória indicadora de falha. $\delta_{i}$ assume valor 1 para o $i$-ésimo indivíduo se $u_{i}=v_{i}=t_{i}$ e caso contrário, $\delta_{i}=0$.

Como se sabe, a distribuição a posteriori é obtida pela normalização do produto da função de verossimilhança e da priori. Portanto, em geral, a distribuição a posteriori de $\theta$ é

$$
\pi(\theta \mid \boldsymbol{t}) \propto L(\boldsymbol{t}, \theta) \pi(\theta) .
$$

Encontrada a distribuição a posteriori de $\theta$, o próximo passo será a estimação dos parâmetros de interesse. Para a estimação dos parâmetros, são utilizadas, por meio do método de MetropolisHastings (MH), as médias a posteriori. Entretanto, o maior interesse deste trabalho está na estimação da curva de sobrevivência. Polpo et al. [2009] consideram as curvas de sobrevivência pelos estimadores plug-in, o que torna impossível a construção de uma banda de credibilidade para a mesma. Portanto, no presente trabalho, a função de sobrevivência será estimada via MH. Este procedimento pode ser resumido no seguinte algoritmo.

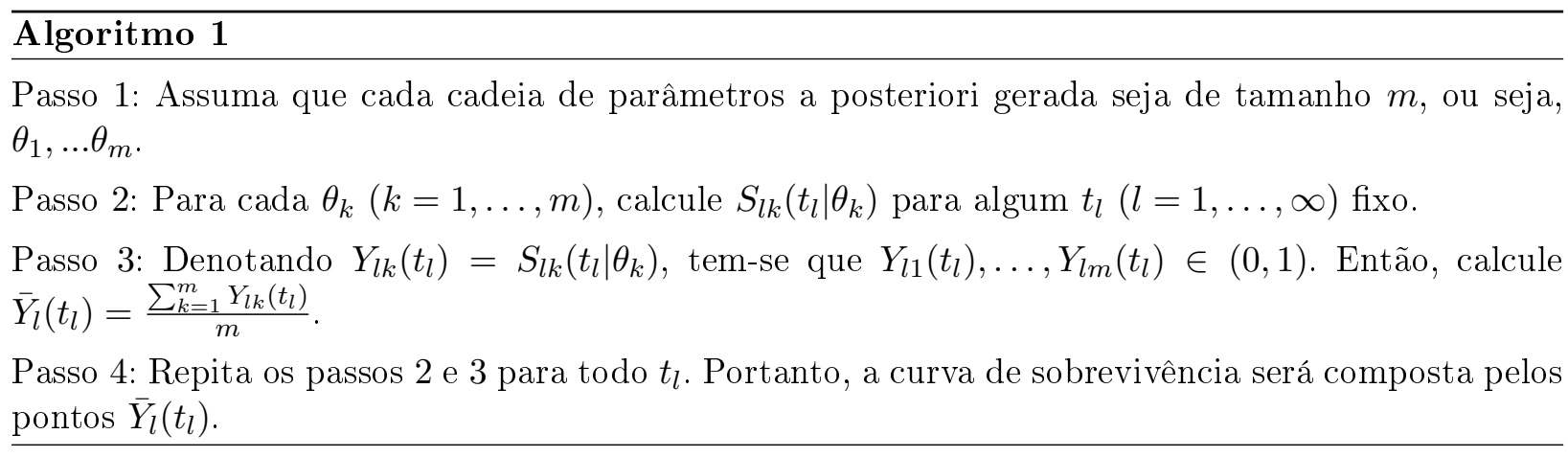

Para se encontar os intervalos de credibilidade para a curva de sobrevivência, basta calcular o primeiro e o terceiro quartis no Passo 3 no lugar de $\bar{Y}_{l}\left(t_{l}\right)$.

Até agora foram apenas mostrados como realizar uma análise de sobrevivência, mas, o objetivo deste estudo é realizar uma metanálise para dados de sobrevivência. Na próxima seção, será mostrada como efetuar uma metanálise com dados referentes a tempo e, então, encontrar a curva de sobrevivência metanalítica.

\subsection{Metanálise para dados de sobrevivência}

A medida metanalítica aqui utilizada é a mesma proposta no Capítulo 2. Como visto, esta medida baseia-se na mistura das distribuições a posteriori da característica de interesse de cada 
estudo incluído na metanálise. A seguir, a construção da distribuição metanalítica do parâmetro em questão será revisada para melhor entendimento.

Considere uma metanálise de $J$ estudos independentes que investigam certa característica de interesse $\theta$. Para o $j$-ésimo estudo $(j=1, \ldots J)$, suponha que $\mathbf{T}_{j}=\left(T_{1, j}, T_{2, j}, \ldots, T_{n_{j}, j}\right)$ denota um vetor transposto de variáveis aleatórias contínuas e não negativas de tamanho $n_{j}$, com função densidade de probabilidade $f\left(\boldsymbol{t}_{j}, \theta\right)$ no espaço de probabilidade $(\mathcal{T}, \mathcal{F}, \mathcal{P})$. Considerando $\boldsymbol{t}_{j}=\left(t_{1, j}, t_{2, j}, \ldots, t_{n_{j}, j}\right)$ um vetor aleatório observado de $\mathbf{T}_{j}$, a função de verossimilhança para o $j$-ésimo estudo é da forma (3.5), denotada por $L_{j}\left(\boldsymbol{t}_{j}, \theta\right)$. Admitindo a metodologia bayesiana, são observadas, respectivamente, sua função a priori de $\theta$ e a distribuição a posteriori de $\theta, \pi(\theta)$ e $\pi_{j}\left(\theta \mid \boldsymbol{t}_{j}\right) \propto L_{j}\left(\boldsymbol{t}_{j}, \theta\right) \pi(\theta)$. Note que a função a priori é a mesma para todos os estudos, porque independe de onde o experimento foi conduzido. Dessa forma, a medida metanalítica chamada de distribuição a posteriori metanalítica de $\theta$, é dada por:

$$
\pi_{M}(\theta \mid \boldsymbol{t})=\sum_{j=1}^{J} \omega_{j} \pi_{j}\left(\theta \mid \boldsymbol{t}_{j}\right) \propto\left[\sum_{j=1}^{J} w_{j} L_{j}\left(\boldsymbol{t}_{j}, \theta\right)\right] \pi(\theta),
$$

em que $\omega_{j}=\frac{n_{j}}{\sum_{j=1}^{J} n_{j}}$.

Este método permite que se obtenha a medida metanalítica de $\theta$. Entretanto, é preciso encontrar a medida metanalítica para a curva de sobrevivência. Então, neste caso, a proposta é encontrar a curva de sobrevivência como na seção anterior, utilizando $\theta$ metanalítico. Para simplificar, considere o algoritmo a seguir para a obtenção da curva de sobrevivência metanalítica.

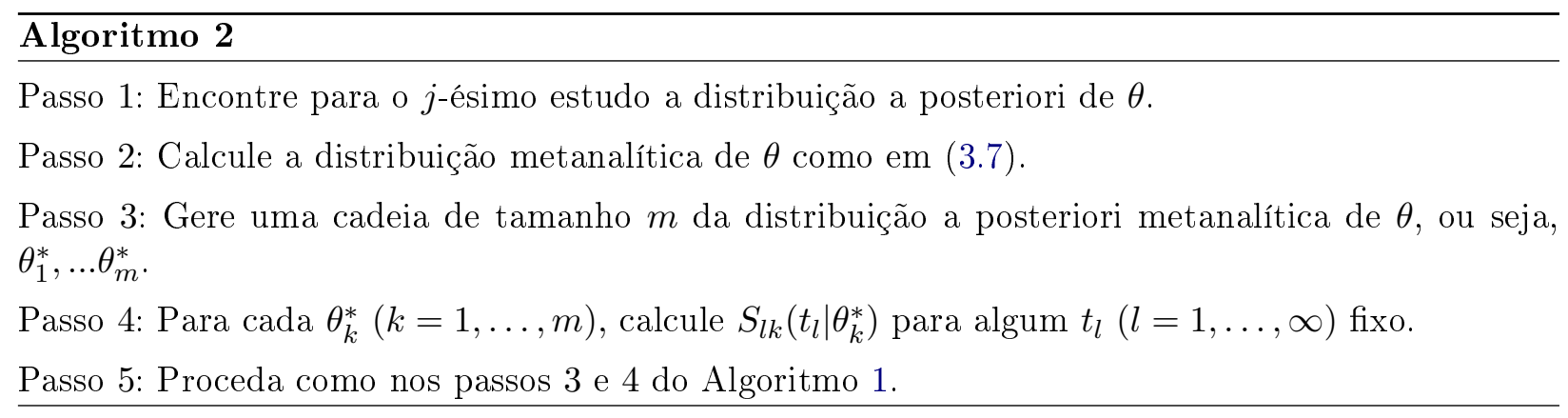

Na próxima seção, alguns exemplos serão mostrados para melhor entendimento.

\subsection{Aplicação}

\subsubsection{Exemplo 1: Modelo Exponencial}

A distribuição Exponencial é, em termos matemáticos, o modelo probabilístico mais simples para descrever dados de sobrevivência. Esta distribuição apresenta apenas um parâmetro, sendo a única que possui função de risco constante, ou seja, tanto uma unidade velha como uma nova tem o mesmo risco de falhar em um intervalo de tempo futuro. Ela é utilizada para descrever o tempo de vida de certos equipamentos e o tempo de vida de pacientes com leucemia por Cox e Snell [1981] (Colosimo e Giolo [2006]).

Para uma metanálise composta de $J$ estudos, a função densidade, de sobrevivência e de risco para a variável aleatória contínua e positiva, tempo de vida, $T$, com distribuição Exponencial são, para o $j$-ésimo estudo, respectivamente,

$$
\begin{gathered}
f_{j}(t, \theta)=\frac{1}{\theta} \exp \left\{-\left(\frac{t}{\theta}\right)\right\}, \\
S_{j}(t, \theta)=\exp \left\{-\left(\frac{t}{\theta}\right)\right\}
\end{gathered}
$$




$$
h_{j}(t, \theta)=\frac{1}{\theta},
$$

em que $j=1,2, \ldots, J$ e o parâmetro $\theta>0$ é o tempo médio de vida. A média da distribuição Exponencial é igual a $E[t]=\theta$ e variância é igual a $\operatorname{Var}[t]=\theta^{2}$.

Para simplificar, considere um exemplo simulado de uma metanálise para dados completos de sobrevivência composta de dois estudos $(J=2)$. Suponha que a variável aleatória de interesse, tempo de vida, é distribuida exponencialmente com parâmetro $\theta$, ou seja, $T \sim \operatorname{Exp}(\theta)$. Os dados do estudo 1 foram gerados de uma distribuição Exponencial com média 4, ou seja, $T_{1} \sim \operatorname{Exp}(4) \mathrm{e}$ o estudo 2 de uma distribuição Exponencial com média 4,2, $T_{2} \sim \operatorname{Exp}(4,2)$. Ambos com tamanho amostral igual a $100,\left(n_{1}=n_{2}=100\right)$. Os dados estão dispostos na Tabela A.3. Pelo histograma (Figura 3.1(a) ), observa-se a distribuição dos tempos de vida referente ao estudo 1 e sua similaridade com a distribuição Exponencial representada pela linha contínua. O mesmo pode ser observado para os dados do estudo 2, Figura 3.1(b). As medidas-resumo dos tempos de vida, para ambos os estudos, encontram-se na Tabela 3.1.

(a) Estudo 1

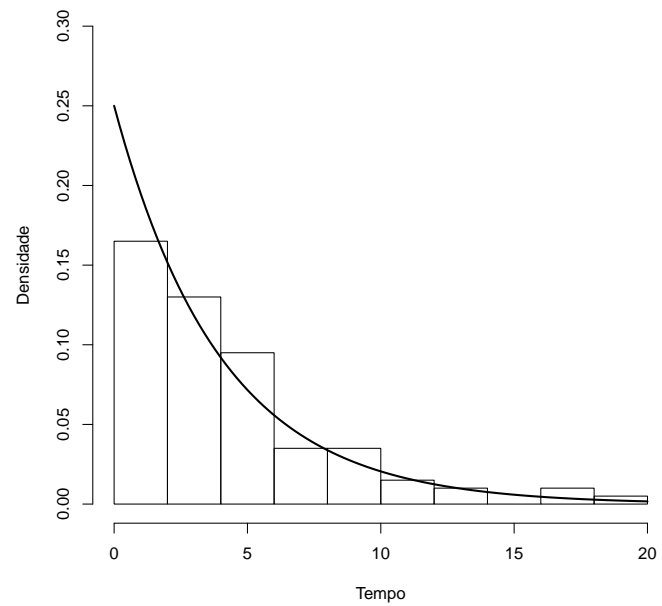

(b) Estudo 2

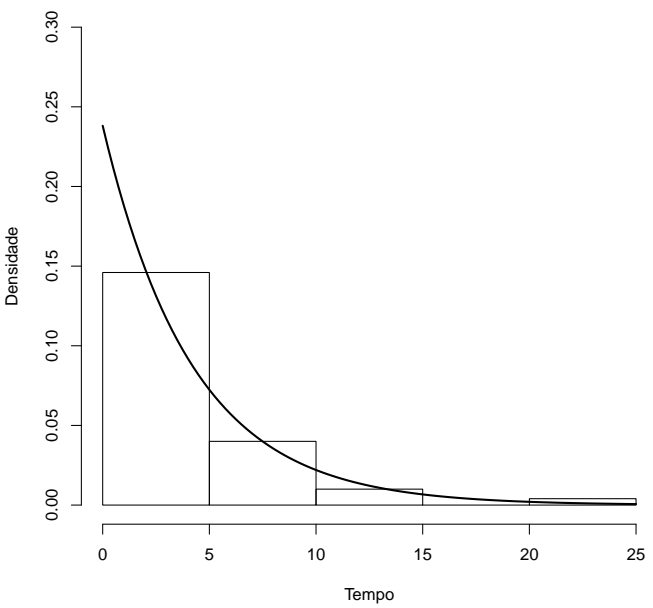

Figura 3.1: (a) Distribuição do tempo de vida do estudo 1. (b) Distribuição do tempo de vida do estudo 2.

Tabela 3.1: Medidas-resumo do tempo de vida de ambos os estudos.

\begin{tabular}{lccccc}
\hline & Tamanho amostral $(n)$ & Média & Mediana & Desvio-Padrão & Soma $\left(\sum_{i=1}^{n_{j}} t_{i j}\right)$ \\
\hline Estudo 1 & 100 & 4,25 & 3,17 & 3,85 & 425,09 \\
Estudo 2 & 100 & 3,86 & 2,52 & 4,12 & 386,75 \\
\hline
\end{tabular}

A função de verossimilhança $\left(L_{j}\left(\boldsymbol{t}_{j}, \theta\right)\right)$ para o $j$-ésimo estudo é

$$
L_{j}\left(\boldsymbol{t}_{j}, \theta\right)=\prod_{i=1}^{n_{j}} \frac{1}{\theta} \exp \left\{-\left(\frac{t_{i j}}{\theta}\right)\right\}=\left(\frac{1}{\theta}\right)^{n_{j}} \exp \left\{-\left(\frac{\sum_{i=1}^{n_{j}} t_{i j}}{\theta}\right)\right\} .
$$

Assumindo que a priori de $\theta$ é a priori não informativa de Jeffreys, $\pi(\theta) \propto \frac{1}{\theta}$, e a função de verossimilhança em (3.11), a distribuição a posteriori de $\theta$, para o $j$-ésimo estudo, é dada por:

$$
\pi_{j}\left(\theta \mid \boldsymbol{t}_{j}\right) \propto\left(\frac{1}{\theta}\right)^{n_{j}+1} \exp \left\{-\left(\frac{\sum_{i=1}^{n_{j}} t_{i j}}{\theta}\right)\right\} .
$$


A distribuição a posteriori de $\theta$, em ambos os estudos, segue distribuição Gama Inversa com parâmetros $n_{j}$ e $\sum_{i=1}^{n_{j}} t_{i j}$. Ou seja, $\theta \mid \boldsymbol{t}_{1} \sim G I(100 ; 425,09)$ e $\theta \mid \boldsymbol{t}_{2} \sim G I(100 ; 386,75)$. Vale ressaltar que a distribuição a posteriori de $\theta$ depende dos dados apenas por meio das estatísticas suficientes $\left(n_{j}, \sum_{i=1}^{n_{j}} t_{i j}\right)$. Assim, tem-se um caso particular cujos resultados obtidos via metanálise caso a caso são equivalentes aos obtidos pela metanálise baseada na literatura. O intervalo HPD de $95 \%$ de credibilidade, média $\left(E\left[\theta \mid t_{j}\right]=\frac{\sum_{i=1}^{n_{j}} t_{i j}}{n_{j}-1}, n_{j}>1\right)$ e variância a posteriori de $\theta$ $\left(\operatorname{Var}\left[\theta \mid t_{j}\right]=\frac{\sum_{i=1}^{n_{j}} t_{i j}^{2}}{\left(n_{j}-1\right)^{2}\left(n_{j}-2\right)}, n_{j}>2\right)$, para ambos os estudos, estão na Tabela 3.2. O interesse deste trabalho está nas curvas de sobrevivência de cada estudo, que são encontradas por meio do procedimento descrito no Algoritmo 1. As cadeias para $\theta$ foram geradas, via $\mathrm{MH}$, com tamanho $m=50.000$, burn in igual a 10.000 e salto de 3, em ambos os estudos. A Figura 3.2 contém as curvas de sobrevivência estimadas para cada estudo. Observe que estas curvas estão muito próximas das verdadeiras, evidenciando um bom ajuste.

Tabela 3.2: Medidas-resumo a posteriori de $\theta$ para ambos os estudos.

\begin{tabular}{lccc}
\hline & $E\left[\theta \mid \boldsymbol{t}_{\boldsymbol{j}}\right]$ & $\operatorname{Var}\left[\theta \mid \boldsymbol{t}_{\boldsymbol{j}}\right]$ & Intervalo HPD de $95 \%$ de credibilidade \\
\hline Estudo 1 & 4,29 & 0,19 & {$[3,47 ; 5,16]$} \\
Estudo 2 & 3,90 & 0,15 & {$[3,16 ; 4,69]$} \\
\hline
\end{tabular}
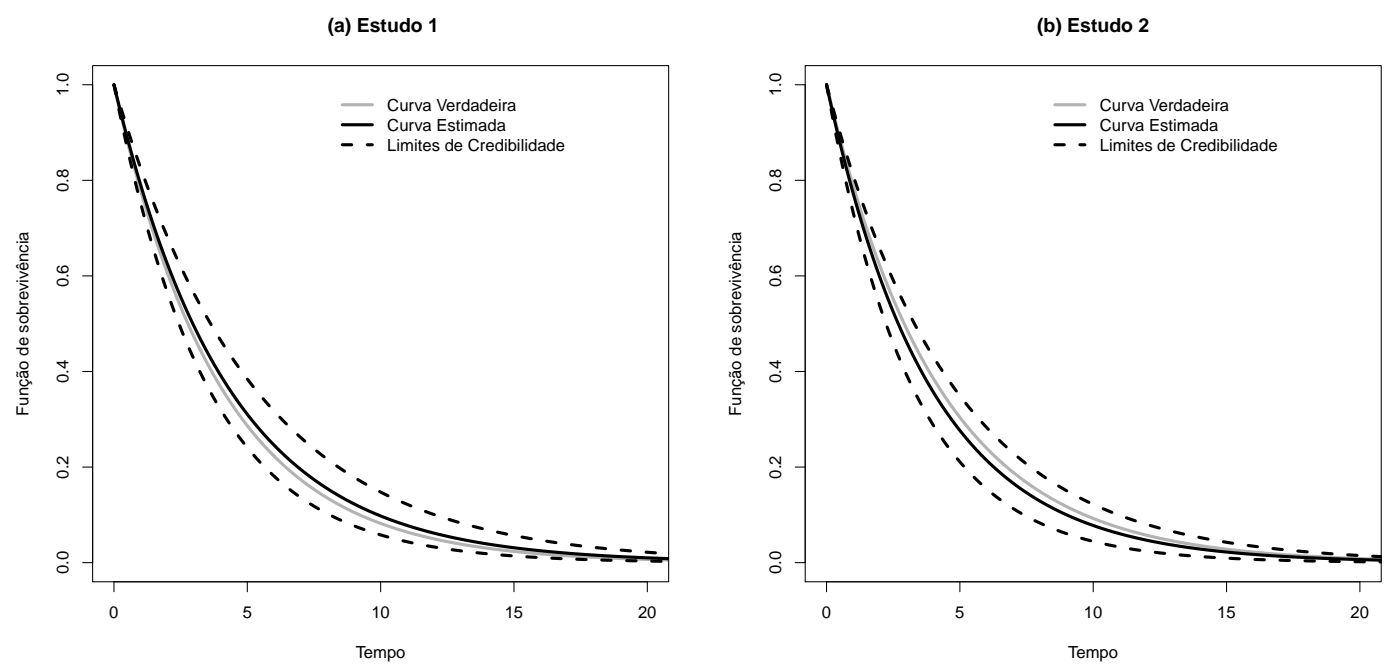

Figura 3.2: Curvas de sobrevivência para o Estudo 1 (a) e para o Estudo 2 (b).

A regra da metanálise é sumarizar os resultados obtidos em cada estudo pertencente à sistematização, permitindo conclusões mais robustas. Então, é preciso obter uma única curva de sobrevivência que resuma as curvas pertencentes a outros estudos. Para tal, primeiramente, deve-se obter a distribuição metanalítica de $\theta$ dada em (3.7).

Baseada em (3.7) e considerando $w_{1}=w_{2}=0,5$, a distribuição a posteriori metanalítica de $\theta$, $\pi_{M}(\theta \mid \boldsymbol{t})$, é dada por:

$$
\pi_{M}(\theta \mid \boldsymbol{t}) \propto \frac{1}{2}\left(\frac{1}{\theta}\right)^{n_{1}+1} \exp \left\{-\left(\frac{\sum_{i=1}^{n_{1}} t_{i 1}}{\theta}\right)\right\}+\frac{1}{2}\left(\frac{1}{\theta}\right)^{n_{2}+1} \exp \left\{-\left(\frac{\sum_{i=1}^{n_{2}} t_{i 2}}{\theta}\right)\right\} .
$$

Utilizando (3.13), calcula-se a curva de sobrevivência metanalítica reportada no Algoritmo 2. A Figura 3.3(a) mostra as distribuições a posteriori de $\theta$ para cada estudo e a distribuição a posteriori 
metanalítica de $\theta$. A média a posteriori metanalítica de $\theta$ é igual a 4,10 , sua variância é igual a 0, 0851 e o intervalo HPD de $95 \%$ de credibilidade é igual a $[3,24 ; 5,01]$. A Figura 3.3(b) contém todas as curvas de sobrevivência para este problema. Observe que todas as curvas pertencem à banda de credibilidade da curva de sobrevivência metanalítica.

Pela curva de sobrevivência, pode-se calcular, por exemplo, a probabilidade de um indivíduo sobreviver mais que o tempo 5 no estudo 1 , estudo 2 e metanálise, ou seja, $S_{1}(t>5)=0,311$, $S_{2}(t>5)=0,277$ e $S_{M}(t>5)=0,294$.

(a)

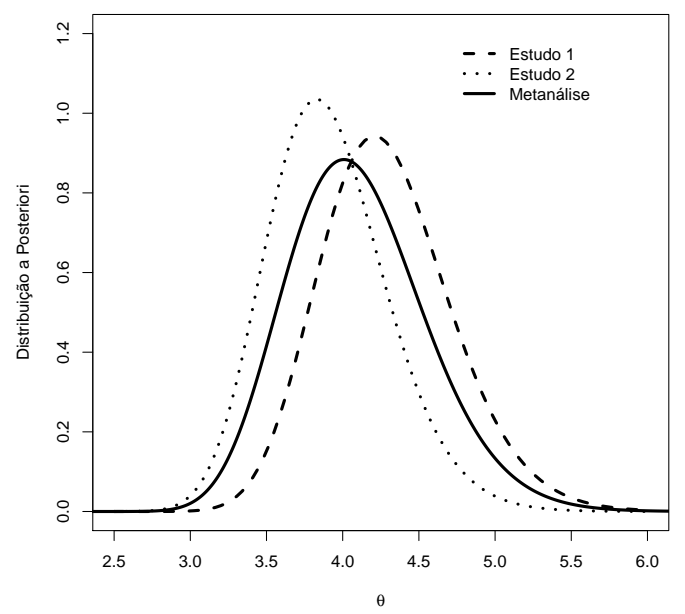

(b)

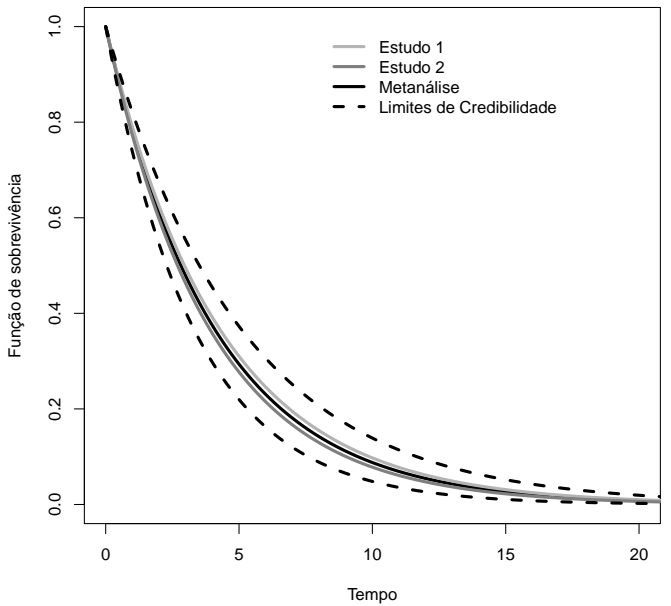

Figura 3.3: (a) Distribuições a posteriori de $\theta$. (b) Curva de sobrevivência metanalítica.

Assuma agora, que as observações são censuradas à direita. Se uma observação for censurada à direita, toda a informação estará resumida no conhecimento de que o tempo de sobrevivência é maior que o tempo registrado. Pode-se representar o mecanismo de censura à direita como um caso especial da censura intervalar. Neste caso, o intervalo $\left[U_{i}, V_{i}\right]$ poderá ser escrito como $\left[U_{i}, \infty\right]$, e a função de verossimilhança dada em (3.5) poderá ser reescrita para dados censurados à direita, como

$$
L(\boldsymbol{t}, \theta)=\prod_{i=1}^{n}\left[f\left(t_{i}, \theta\right)\right]^{\delta_{i}}\left[S\left(t_{i}, \theta\right)\right]^{1-\delta_{i}}
$$

onde $\delta_{i}=1$ se $t_{i}$ é exato e $\delta_{i}=0$ se $t_{i}$ é censurado à direita.

Suponha uma metanálise para dados de sobrevivência censurados à direita seja composta de dois estudos. Os dados dos estudos são os mesmos do exemplo anterior, entretanto, gerou-se a variável indicadora de falha para cada indivíduo pertencente à metanálise. Os números de censuras nos estudo 1 e 2 são, respectivamente, 11 e 16, ou seja, $\sum_{i=1}^{n_{1}} \delta_{i 1}=89$ e $\sum_{i=1}^{n_{2}} \delta_{i 2}=84$. A função de verossimilhança para o $j$-ésimo estudo é

$$
L_{j}\left(\boldsymbol{t}_{j}, \theta\right)=\prod_{i=1}^{n_{j}}\left[\frac{1}{\theta} \exp \left\{-\frac{t_{i j}}{\theta}\right\}\right]^{\delta_{i j}}\left[\exp \left\{-\frac{t_{i j}}{\theta}\right\}\right]^{1-\delta_{i j}}=\left[\frac{1}{\theta}\right]^{\sum_{i=1}^{n_{j}} \delta_{i j}} \exp \left\{-\frac{\sum_{i=1}^{n_{j}} t_{i j}}{\theta}\right\} .
$$

Considerando a priori de Jeffreys $\pi(\theta) \propto \frac{1}{\theta}$ e a função de verossimilhança em (3.15), a distribuição a posteriori de $\theta$ para o $j$-ésimo estudo é dada por:

$$
\pi_{j}\left(\theta \mid \boldsymbol{t}_{j}\right) \propto\left[\frac{1}{\theta}\right]^{\left(\sum_{i=1}^{n_{j}} \delta_{i j}\right)+1} \exp \left\{-\frac{\sum_{i=1}^{n_{j}} t_{i j}}{\theta}\right\} .
$$

Logo, a distribuição a posteriori de $\theta$, em ambos os estudos, segue distribuição Gama Inversa com parâmetros $\sum_{i=1}^{n_{j}} \delta_{i j}$ e $\sum_{i=1}^{n_{j}} t_{i j}$, ou seja, $\theta \mid \boldsymbol{t}_{1} \sim G I(89 ; 425,09)$ e $\theta \mid \boldsymbol{t}_{2} \sim G I(84 ; 386,75)$. 
Novamente, nota-se que a distribuição a posteriori de $\theta$ depende dos dados por meio das estatísticas suficientes $\left(\sum_{i=1}^{n_{j}} t_{i j} ; \sum_{i=1}^{n_{j}} \delta_{i j}\right)$. O intervalo HPD de $95 \%$ de credibilidade, a média e a variância a posteriori de $\theta$, para ambos os estudos, encontram-se na Tabela 3.3. Entretanto, o maior interesse está na estimação da curva de sobrevivência para cada estudo e, consequentemente, encontrar a curva de sobrevivência metanalítica. A Figura 3.4 contém as curvas de sobrevivência estimadas para cada estudo, como no Algoritmo 1. As cadeias a posteriori de $\theta$ foram geradas, via MH, com tamanho $m=50.000$, burn in igual a 10.000 e salto de 3 , em ambos os estudos. Note que, no Estudo 1, a curva de sobrevivência estimada está um pouco distante da real. No entanto, a verdadeira curva de sobrevivência encontra-se dentro da banda de $95 \%$ de credibilidade. Apesar da presença de censura, a curva estimada do Estudo 2 está próxima da curva verdadeira.

Tabela 3.3: Medidas-resumo a posteriori de $\theta$ para ambos os estudos com dados censurados.

\begin{tabular}{lccc}
\hline & $E\left[\theta \mid \boldsymbol{t}_{\boldsymbol{j}}\right]$ & $\operatorname{Var}\left[\theta \mid \boldsymbol{t}_{\boldsymbol{j}}\right]$ & Intervalo HPD de $95 \%$ de credibilidade \\
\hline Estudo 1 & 4,83 & 0,27 & {$[3,86 ; 5,86]$} \\
Estudo 2 & 4,65 & 0,26 & {$[3,69 ; 5,68]$} \\
\hline
\end{tabular}
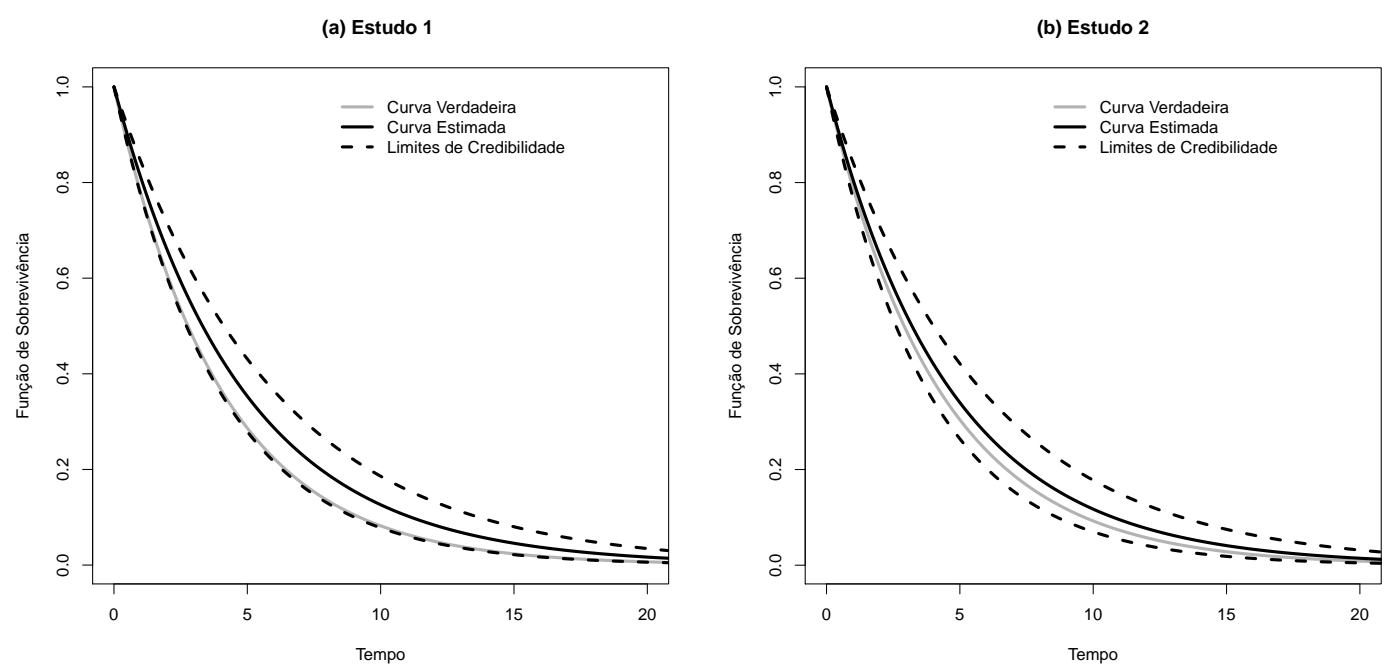

Figura 3.4: Curvas de sobrevivência relacionadas aos estudos 1 (a) e 2 (b), respectivamente.

Agora, é preciso obter uma única curva de sobrevivência que sumarize as curvas pertencentes aos estudos. Para tal, primeiramente, deve-se obter a medida metanalítica de $\theta$ dada em (3.7).

Baseada em (3.7) e considerando $w_{1}=w_{2}=0,5$, a distribuição a posteriori metanalítica de $\theta$, $\pi_{M}(\theta \mid \boldsymbol{t})$, é

$$
\pi_{M}(\theta \mid \boldsymbol{t}) \propto \frac{1}{2}\left[\frac{1}{\theta}\right]^{(89)+1} \exp \left\{-\frac{425,09}{\theta}\right\}+\frac{1}{2}\left[\frac{1}{\theta}\right]^{(84)+1} \exp \left\{-\frac{386,75}{\theta}\right\} .
$$

A Figura 3.5(a) mostra as distribuições a posteriori de $\theta$ para cada estudo e sua distribuição a posteriori metanalítica. A média a posteriori do $\theta$ metanalítico é igual a 4,74 , sua variância a posteriori é igual a 0,13 , e o intervalo HPD de $95 \%$ de credibilidade é igual a $[3,76 ; 5,78]$. Baseandose na distribuição metanalítica (3.17), calcula-se a curva de sobrevivência metanalítica reportada no Algoritmo 2. A Figura 3.5(b) contém todas as curvas de sobrevivência para esse problema e todas estas curvas pertencem à banda de credibilidade da curva de sobrevivência metanalítica. A probabilidade de um indivíduo sobreviver mais que o tempo 5 nos estudos 1 e 2 e na metanálise, são respectivamente, $S_{1}(t>5)=0,353, S_{2}(t>5)=0,340$ e $S_{M}(t>5)=0,347$. 
(a)

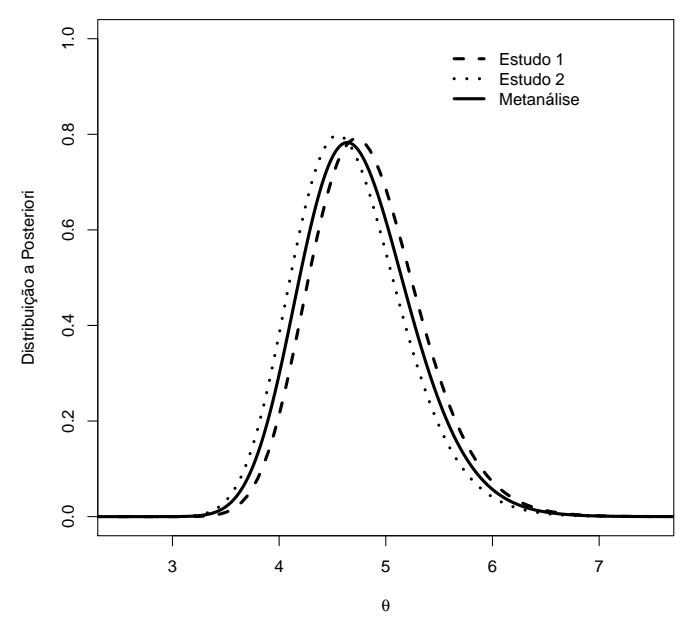

(b)

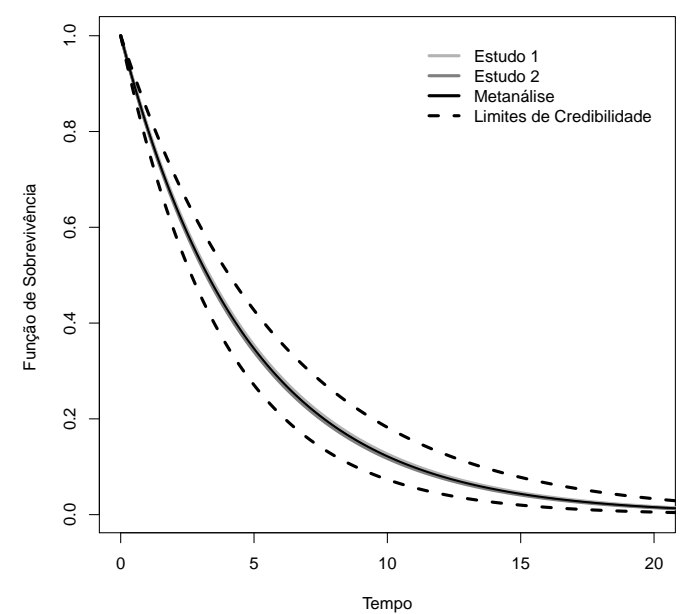

Figura 3.5: (a) Distribuiçỗes a posteriori de $\theta$. (b) Curvas de sobrevivência.

\subsubsection{Exemplo 2: Modelo Log-normal}

A distribuição Log-normal é muito empregada para caracterizar o tempos de vida dos produtos e dos indivíduos. Como o nome sugere, o logaritmo de uma variável com distribuição Log-normal de parâmetros $\mu$ e $\sigma$ tem distribuição Normal com média $\mu$ e desvio-padrão $\sigma$. Esta relação significa que dados provenientes de uma distribuição Log-normal podem ser analisados, conforme uma distribuição Normal se o logaritmo dos dados for trabalhado ao invés dos valores originais (Colosimo e Giolo [2006]).

A função densidade de uma variável aleatória $T$ com distribuição Log-normal é dada por:

$$
f\left(t, \mu, \sigma^{2}\right)=\frac{1}{\sqrt{2 \pi} t \sigma} \exp \left\{-\frac{1}{2}\left(\frac{\log (t)-\mu}{\sigma}\right)^{2}\right\}, t>0,
$$

em que $\mu$ é a média do logaritmo do tempo de falha, assim como $\sigma$ é o desvio-padrão.

As funções de sobrevivência e de taxa de falha de uma variável Log-normal não apresentam uma forma analítica explícita e, desse modo, são representadas, respectivamente, por:

$$
S\left(t, \mu, \sigma^{2}\right)=\Phi\left(\frac{-\log (t)+\mu}{\sigma}\right)
$$

e

$$
h\left(t, \mu, \sigma^{2}\right)=\frac{f(t)}{S(t)},
$$

em que $\Phi(\bullet)$ é a função de distribuição acumulada de uma Normal padrão.

As funções densidade, sobrevivência e risco estão representadas na Figura 3.6(a), Figura 3.6(b) e Figura 3.6(c). A média e a variância da distribuição Log-normal são dadas, respectivamente, por:

$$
E(t)=\exp \left\{\mu+\sigma^{2} / 2\right\}
$$

e

$$
\operatorname{Var}(t)=\exp \left\{2 \mu+\sigma^{2}\right\}\left(\exp \left\{\sigma^{2}\right\}-1\right)
$$


(a)

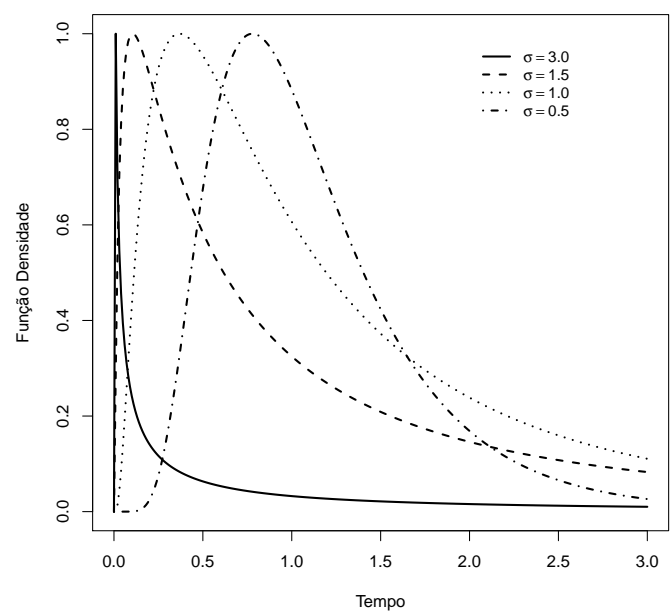

(b)

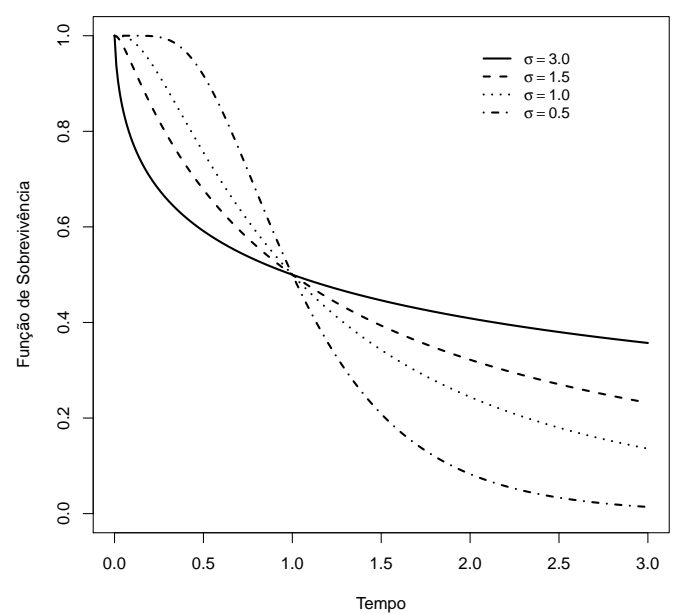

(c)

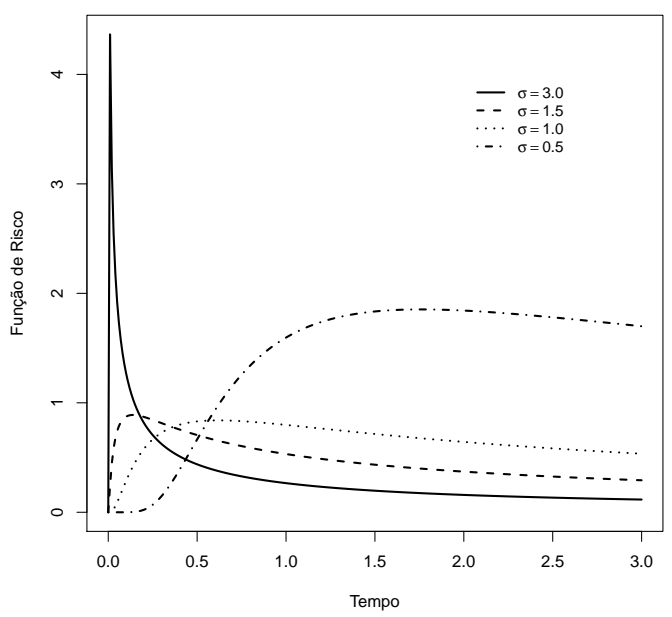

Figura 3.6: (a) Função densidade Log-normal, (b) Função de sobrevivência Log-normal e (c) Função de risco Log-normal.

Considere um exemplo simulado de uma metanálise para dados completos de sobrevivência composta de três estudos $(J=3)$. Suponha que a variável aleatória de interesse, tempo de vida, é distribuída log-normalmente com parâmetros $\mu$ e $\sigma$, ou seja, $T \sim L N(\mu, \sigma)$. Os dados do Estudo 1 foram gerados de uma distribuição Log-normal com parâmetros 2,5 e 1/2, ou seja, $T_{1} \sim L N(2,5 ; 1 / 2)$, o Estudo 2 de uma distribuição Log-normal com parâmetros 3 e $1 / 2, T_{2} \sim L N(3 ; 1 / 2)$ e o Estudo 3 de uma distribuição Log-normal com parâmetros 2,8 e $2 / 3, T_{3} \sim L N(2,8 ; 2 / 3)$. Todos os estudos com tamanho amostral iguais a $40,\left(n_{1}=n_{2}=n_{3}=40\right)$. Os dados estão dispostos na Tabela A.1 e as medidas-resumo (mínimo, primeiro quartil (Q1), mediana, média, terceiro quartil (Q3), máximo e desvio-padrão) dos tempos de vida, para todos os estudos, encontram-se na Tabela 3.4.

Tabela 3.4: Medidas-resumo do tempo de sobrevivência em cada estudo pertencente à metanálise.

\begin{tabular}{lccccccc}
\hline & Mínimo & Q1 & Mediana & Média & Q3 & Máximo & Desvio-padrão \\
\hline Estudo 1 & 4,30 & 10,10 & 13,75 & 14,72 & 17,15 & 39,10 & 6,93 \\
Estudo 2 & 7,10 & 16,08 & 19,10 & 21,56 & 27,60 & 65,60 & 10,22 \\
Estudo 3 & 3,30 & 10,25 & 13,75 & 18,53 & 23,48 & 70,20 & 13,56 \\
\hline
\end{tabular}


(a) Estudo 1

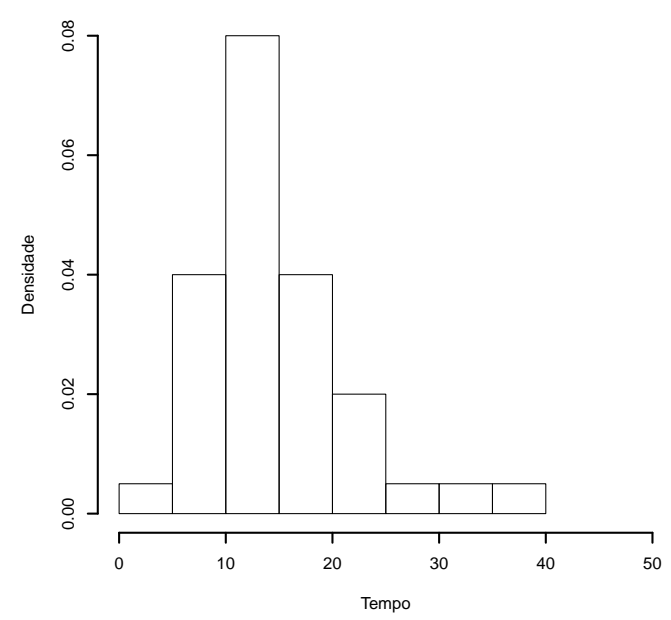

(b) Estudo 2

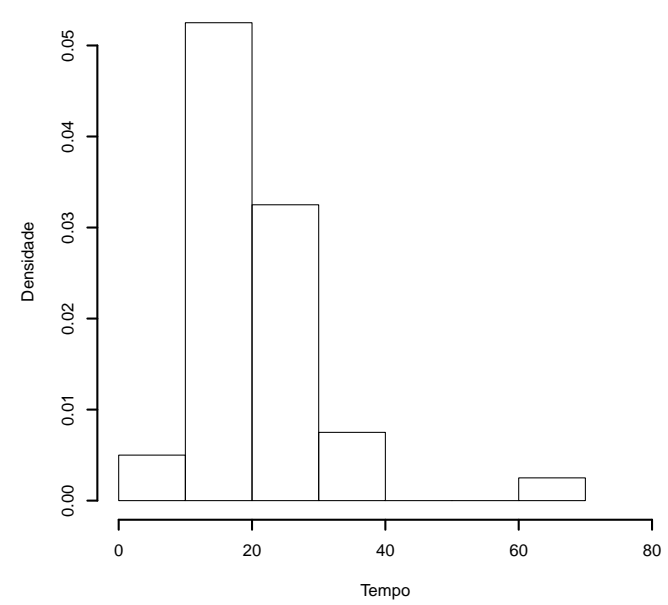

(c) Estudo 3

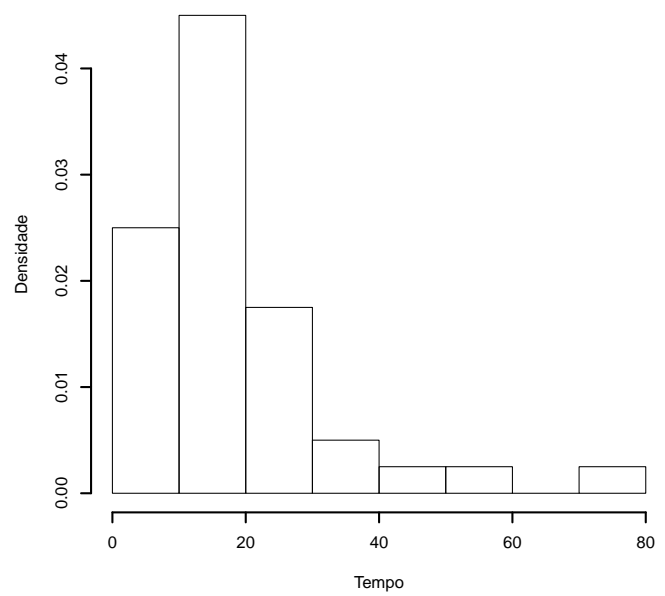

Figura 3.7: (a) Distribuição dos tempos de vida do Estudo 1. (b) Distribuição dos tempos de vida do Estudo 2. (c) Distribuição dos tempos de vida do Estudo 3.

Utilizando o fato que, se $T \sim L N(\mu, \sigma), Y=\log (T) \sim N(\mu, \sigma)$, o problema torna-se simples. Então, a partir de agora, considera-se que o modelo é Normal. Logo, a função de verossimilhança para o $j$-ésimo estudo é dada por:

$$
L_{j}\left(\boldsymbol{y}_{j}, \mu, \sigma^{2}\right) \propto\left(\sigma^{2}\right)^{-n_{j} / 2} \exp \left\{-\frac{1}{2 \sigma^{2}}\left[\left(n_{j}-1\right) s_{j}^{2}+n_{j}\left(\mu-\bar{y}_{j}\right)^{2}\right]\right\},
$$

onde $n_{j}$ é o tamanho amostral do $j$-ésimo estudo, $\bar{y}_{j}$ é a média amostral e $s_{j}^{2}$, a variância amostral do $j$-ésimo estudo.

Considerando a priori de Jeffreys $\pi\left(\mu, \sigma^{2}\right) \propto\left(\sigma^{2}\right)^{-1}$ e a função de verossimilhança em (3.23), a distribuição a posteriori de $\mu$ e $\sigma^{2}$ em todos os estudos segue distribuição Normal-Gama-Inversa, dada por:

$$
\pi_{j}\left(\mu, \sigma^{2} \mid \boldsymbol{y}_{j}\right)=\frac{\sqrt{n_{j}}}{\sigma \sqrt{2 \pi}} \frac{\left[\left(n_{j}-1\right) s_{j}^{2} / 2\right]^{n_{j} / 2}}{\Gamma\left(n_{j} / 2\right)}\left(\frac{1}{\sigma^{2}}\right)^{\frac{n_{j}+1}{2}+1} \exp \left\{-\frac{\left(n_{j}-1\right) s_{j}^{2}+n_{j}\left(\mu-\bar{y}_{j}\right)^{2}}{2 \sigma^{2}}\right\} .
$$

Novamente, a distribuição a posteriori de $\left(\mu, \sigma^{2}\right)$ depende dos dados apenas por meio das estatísticas suficientes $\left(n_{j}, \bar{y}_{j}, s_{j}^{2}\right)$. Os valores esperados a posteriori e a matriz de variância-covariância 
a posteriori de $\left(\mu, \sigma^{2}\right)$ para cada estudo são, respectivamente,

$$
\begin{gathered}
E\left[\mu, \sigma^{2} \mid \boldsymbol{y}\right]=\left(\begin{array}{ccc}
\bar{y}_{1} & \bar{y}_{2} & \bar{y}_{3} \\
\frac{\left(n_{1}-1\right) s_{1}^{2}}{\left(n_{1}-3\right)} & \frac{\left(n_{2}-1\right) s_{2}^{2}}{\left(n_{2}-3\right)} & \frac{\left(n_{3}-1\right) s_{3}^{2}}{\left(n_{3}-3\right)}
\end{array}\right)=\left(\begin{array}{ccc}
2,59 & 2,98 & 2,71 \\
0,22 & 0,19 & 0,44
\end{array}\right), n_{j}>3 \\
\operatorname{Cov}\left[\mu, \sigma^{2} \mid \boldsymbol{y}_{1}\right]=\left[\begin{array}{cc}
\frac{s_{1}^{2}\left(n_{1}-1\right)}{n_{1}\left(n_{1}-3\right)} & 0 \\
0 & \frac{2 s_{1}^{2}\left(n_{1}-1\right)}{\left(n_{1}-5\right)\left(n_{1}-3\right)^{2}}
\end{array}\right]=\left[\begin{array}{cc}
0,00551 & 0 \\
0 & 0,00034
\end{array}\right], n_{1}>5 \\
\operatorname{Cov}\left[\mu, \sigma^{2} \mid \boldsymbol{y}_{2}\right]=\left[\begin{array}{cc}
\frac{s_{2}^{2}\left(n_{2}-1\right)}{n_{2}\left(n_{2}-3\right)} & 0 \\
0 & \frac{2 s_{2}^{2}\left(n_{2}-1\right)}{\left(n_{2}-5\right)\left(n_{2}-3\right)^{2}}
\end{array}\right]=\left[\begin{array}{cc}
0,0048 & 0 \\
0 & 0,0003
\end{array}\right], n_{2}>5 \\
\operatorname{Cov}\left[\mu, \sigma^{2} \mid \boldsymbol{y}_{3}\right]=\left[\begin{array}{cc}
\frac{s_{3}^{2}\left(n_{3}-1\right)}{n_{3}\left(n_{3}-3\right)} & 0 \\
0 & \frac{2 s_{3}^{2}\left(n_{3}-1\right)}{\left(n_{3}-5\right)\left(n_{3}-3\right)^{2}}
\end{array}\right]=\left[\begin{array}{cc}
0,0110 & 0 \\
0 & 0,0007
\end{array}\right], n_{3}>5 .
\end{gathered}
$$

Após encontrar as distribuições a posteriori de $\left(\mu, \sigma^{2}\right)$ e seus respectivos valores esperados, para cada estudo pertencente à metanálise, o próximo passo será a estimação das curvas de sobrevivência via MH. Para tal, utiliza-se o procedimento descrito no Algoritmo 1. As cadeias para $\mu$ e $\sigma^{2}$ foram geradas com tamanho $m=50.000$, burn in igual a 10.000 e salto de 5. A Figura 3.8 contém as curvas de sobrevivência estimadas para cada estudo. 

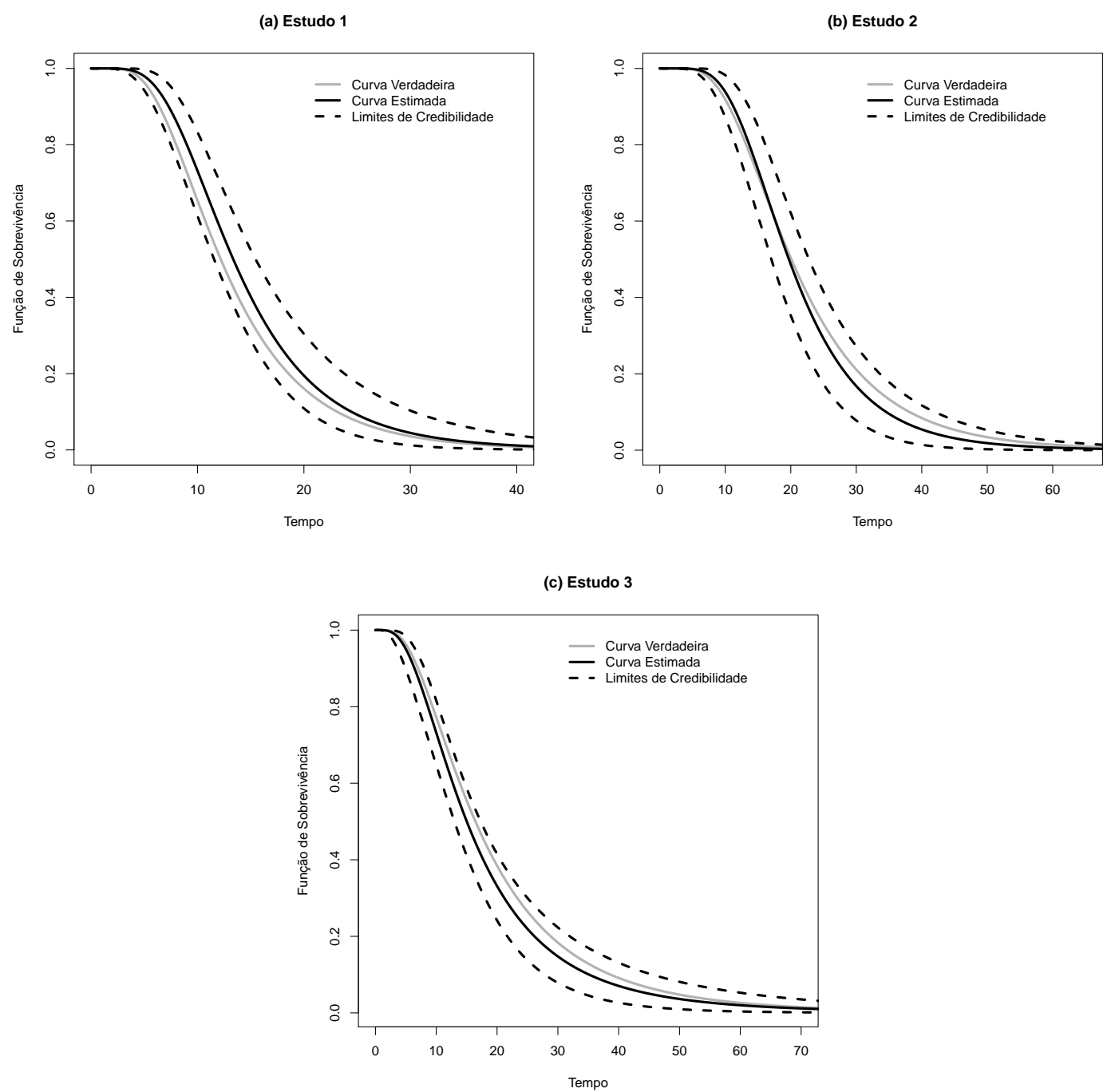

Figura 3.8: Curvas de sobrevivência para o Estudo 1 (a), para o Estudo 2 (b) e para o Estudo 3 (c).

Para se obter uma única curva de sobrevivência que resuma as curvas pertencentes a outros estudos, primeiramente, precisa-se obter a distribuição metanalítica de $\mu$ e $\sigma^{2}$ dada em (3.7).

Considerando $w_{1}=w_{2}=w_{3}=1 / 3$, a distribuição a posteriori metanalítica de $(\mu, \sigma), \pi_{M}(\mu, \sigma \mid \boldsymbol{y})$, é dada por:

$$
\pi_{M}(\mu, \sigma \mid \boldsymbol{y}) \propto \sum_{j=1}^{3} \frac{1}{3} \pi\left(\mu, \sigma^{2} \mid \boldsymbol{y}_{j}\right) .
$$

A Figura 3.9(a) contém as curvas de nível das distribuições a posteriori de $\left(\mu, \sigma^{2}\right)$ para cada estudo e a Figura 3.9(b), as curvas de nível da distribuição a posteriori metanalítica de $\left(\mu, \sigma^{2}\right)$. Na Figura 3.9(c), está o gráfico tridimensional da distribuição a posteriori metanalítica $\left(\mu, \sigma^{2}\right)$. A estimação por região HPD de 95\% de credibilidade está na Figura 3.9(d). Utilizando (3.29), calculase a curva de sobrevivência metanalítica por meio do Algoritmo 2. A Figura 3.10(a) contém a curva de sobrevivência metanalítica e sua banda de 95\% credibilidade. Pela Figura 3.10(b), que contém todas as curvas de sobrevivência para este problema, observa-se que todas as curvas pertencem à banda de credibilidade da curva de sobrevivência metanalítica. Pela curva de sobrevivência, podese calcular, por exemplo, a probabilidade de um indivíduo sobreviver mais que o tempo 20 no estudo 1,2 e metanálise, ou seja, $S_{1}(t>20)=0,195, S_{2}(t>20)=0,486, S_{3}(t>20)=0,331$ e $S_{M}(t>20)=0,337$. 
(a)

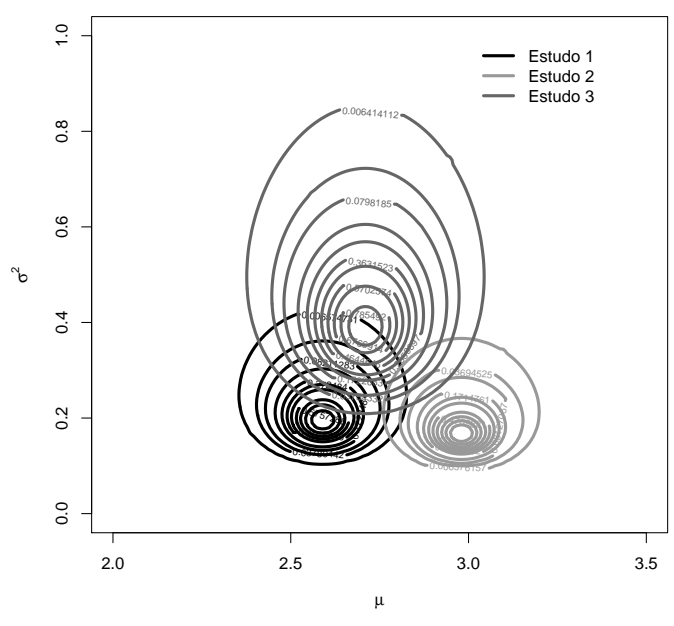

(c)

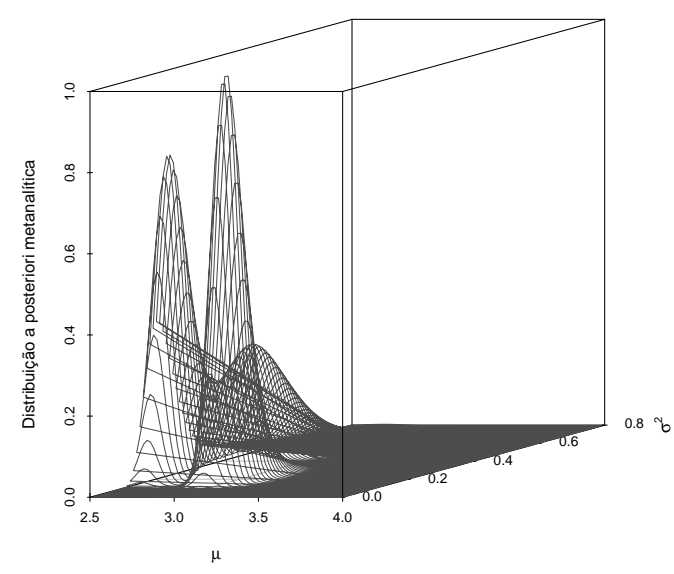

(b)

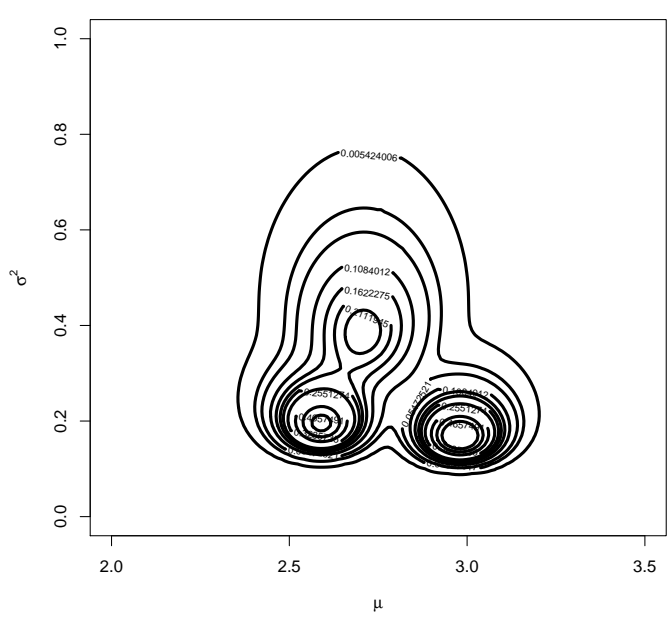

(d)

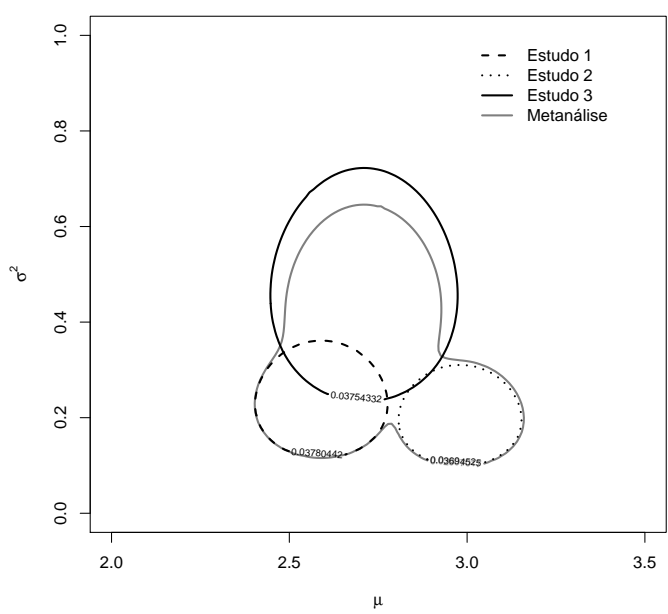

Figura 3.9: (a) Curvas de nivel das distribuições a posteriori de $(\mu, \sigma)$ para cada estudo. (b) Curvas de nivel da distribuição a posteriori metanalítica de $(\mu, \sigma)$. (c) Distribuição a posteriori metanalítica de $(\mu, \sigma)$ em 3D. (d) Região HPD de $95 \%$ de credibilidade.

(a)

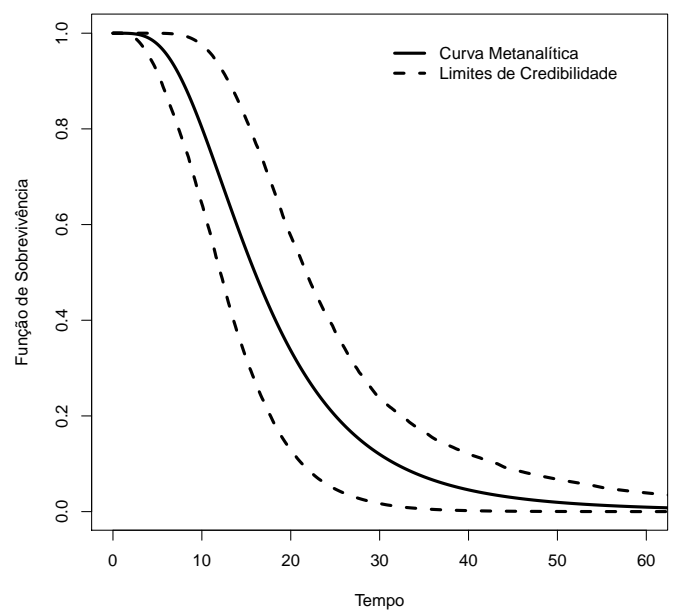

(b)

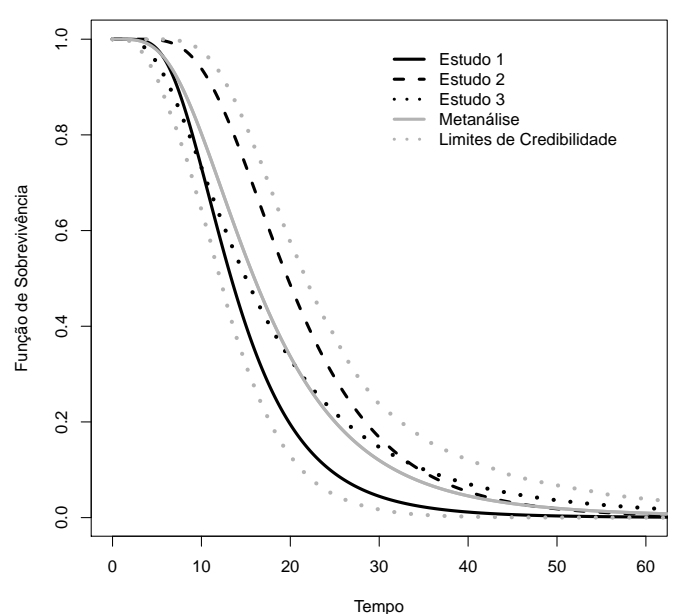

Figura 3.10: (a) Curva de sobrevivência metanalitica. (b) Curvas de sobrevivência para o problema. 


\subsubsection{Exemplo 3: Modelo Weibull}

A distribuição Weibull é amplamente usada em confiabilidade, foi apresentada originalmente por Weibull [1939] e utilizada em confiabilidade, posteriormente por Weibull [1951] e, desde então, vem sendo amplamente utilizada, como modelo em aplicações biométricas, industriais e em muitas outras áreas. Uma das características importantes da distribuição Weibull na modelagem de tempos de sobrevivência ou confiabilidade está relacionada à sua flexibilidade em acomodar diferentes formas na taxa de falha (função de risco), apenas variando os valores de seus parâmetros.

Para o tempo de vida $T \geq 0$, dados os parâmetros de forma $(\beta>0)$ e escala $(\eta>0)$, as funções densidade, de sobrevivência e de risco da distribuição Weibull $(W e i(\beta, \eta))$ são dadas, respectivamente, por:

$$
\begin{gathered}
f(t, \beta, \eta)=\frac{\beta}{\eta^{\beta}} t^{\beta-1} \exp \left[-\left(\frac{t}{\eta}\right)^{\beta}\right], \\
S(t, \beta, \eta)=1-F(t \mid \beta, \eta)=\exp \left[-\left(\frac{t}{\eta}\right)^{\beta}\right]
\end{gathered}
$$

e

$$
h(t, \beta, \eta)=\frac{\beta}{\eta^{\beta}} t^{\beta-1} .
$$

A função de verossimilhança para $n$ dados de sobrevivência, utilizando o modelo Weibull é expressa por

$$
L(\boldsymbol{t}, \beta, \eta)=\prod_{i=1}^{n} \frac{\beta}{\eta^{\beta}} t_{i}^{\beta-1} \exp \left[-\left(\frac{t_{i}}{\eta}\right)^{\beta}\right] .
$$

A média e a variância da distribuição Weibull são dadas por:

$$
E(t \mid \beta, \eta)=\eta \Gamma\left(1+\eta^{-1}\right),
$$

em que $\Gamma(\cdot)$ é a função gama, e

$$
\operatorname{Var}(t \mid \beta, \eta)=\eta^{2}\left[\Gamma\left(1+2 \eta^{-1}\right)-\Gamma\left(1+\eta^{-1}\right)^{2}\right] .
$$

Para se obter uma variedade de comportamentos dessa distribuição, basta alterar o parâmetro de forma $\beta$. Determinados valores desse parâmetro fornecem alguns casos particulares, por exemplo: $\beta=1$ dá a distribuição Exponencial; $\beta=2$, a distribuição Rayleigh, obtém-se uma aproximação das distribuições Log-normal e Normal para $\beta=2,5$ e $\beta=3,6$, respectivamente.

Os comportamentos das funções de densidade, de sobrevivência e de risco do modelo Weibull estão representados nas Figura 3.11(a), Figura 3.11(b) e Figura 3.11(c).

Pela Figura 3.11(b), verifica-se o comportamento da função de sobrevivência Weibull para os diferentes valores de $\beta$. Vale ressaltar que, independente do valor de $\beta$, quando $t=\eta$, tem-se $S(t)=$ $e^{-1}$. Na Figura 3.11(c), são observadas as principais formas da taxa de falha para a distribuição Weibull, que podem ser descritas por $\beta<1$ (taxa de falha decrescente), $\beta=1$ (taxa de falha constante) e $\beta>1$ (taxa de falha crescente). 
(a)

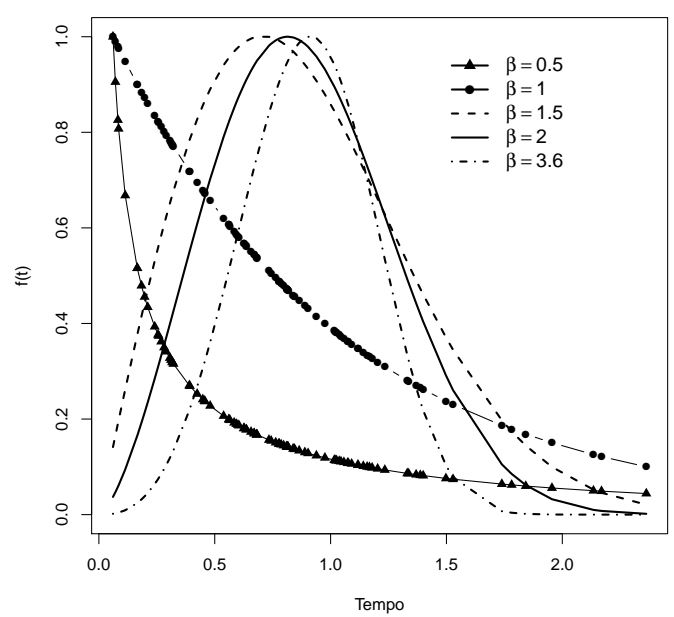

(b)

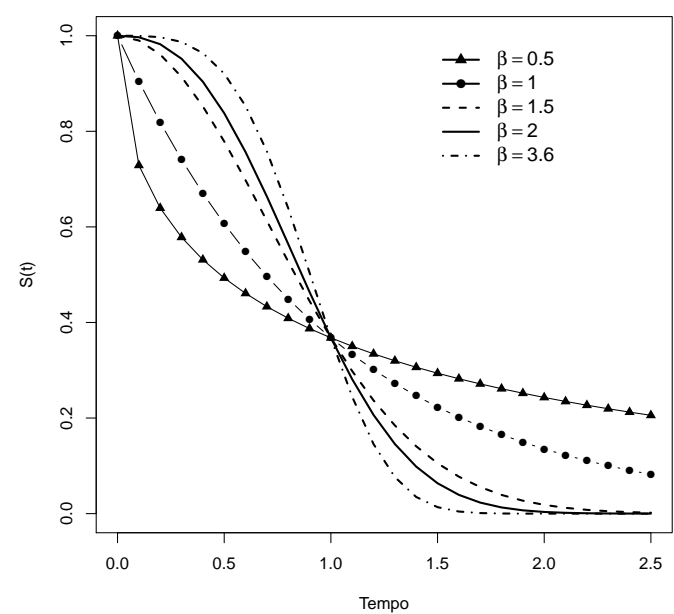

(c)

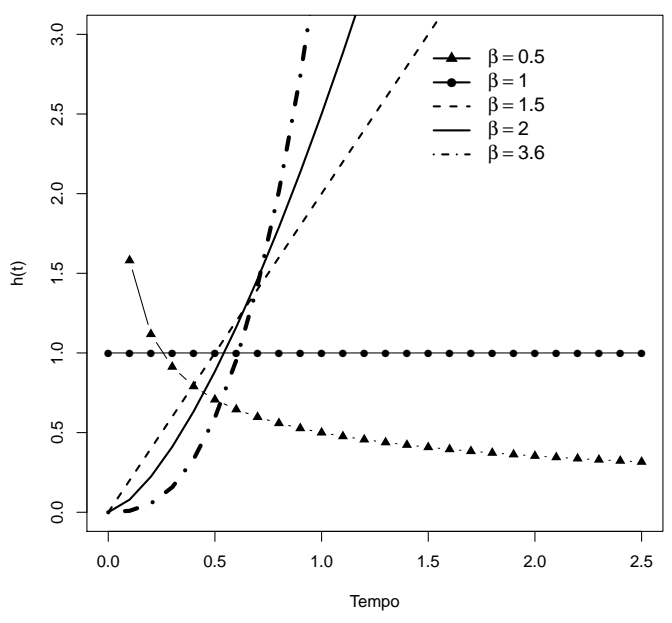

Figura 3.11: (a) Função densidade do modelo Weibull $(\eta=1)$. (b) Função de confiabilidade do modelo Weibull $(\eta=1)$. (c) Função de risco do modelo Weibull $(\eta=1)$.

Considere um exemplo simulado de uma metanálise caso a caso para dados completos de sobrevivência composta de dois estudos. Suponha que a variável aleatória de interesse, tempo de vida, segue distribuição Weibull com parâmetros $\beta$ e $\eta$. Ou seja, $T \sim(W e i(\beta, \eta))$. Os dados do Estudo 1 foram gerados de uma distribuição Weibull com parâmetros 2 e 1 , ou seja, $\boldsymbol{t}_{1} \sim($ Wei $(2,1))$ e o Estudo 2 de uma distribuição Weibull com parâmetros 1 e $1, \boldsymbol{t}_{2} \sim(W e i(1,1))$. Ambos os estudos com tamanho amostral igual a $50,\left(n_{1}=n_{2}=50\right)$. Os dados estão dispostos na Tabela A.2 e as medidas-resumo (mínimo, primeiro quartil (Q1), mediana, média, terceiro quartil (Q3), máximo e desvio-padrão) dos tempos de vida, para todos os estudos, encontram-se na Tabela 3.5.

Tabela 3.5: Medidas-resumo do tempo de sobrevivência para ambos os estudos pertencentes à metanálise.

\begin{tabular}{lccccccc}
\hline & Mínimo & Q1 & Mediana & Média & Q3 & Máximo & Desvio-padrão \\
\hline Estudo 1 & 0,15 & 0,44 & 0,74 & 0,85 & 1,15 & 2,15 & 0,52 \\
Estudo 2 & 0,01 & 0,39 & 0,85 & 1,02 & 1,30 & 4,27 & 0,97 \\
\hline
\end{tabular}


(a) Estudo 1

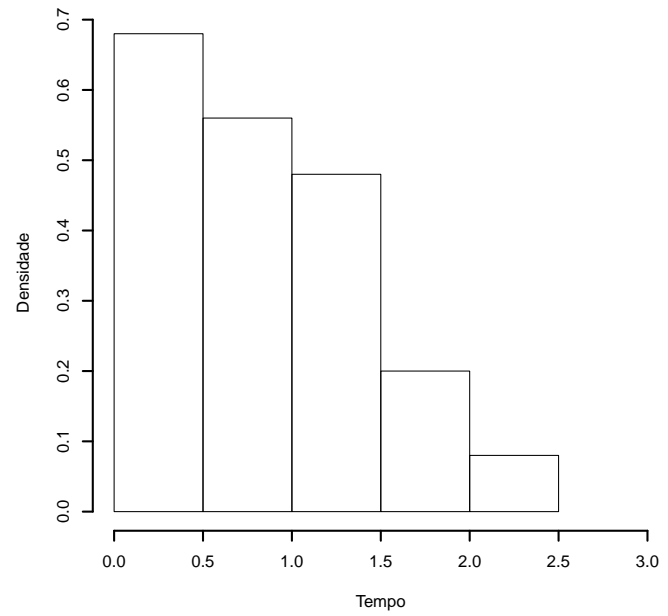

(b) Estudo 2

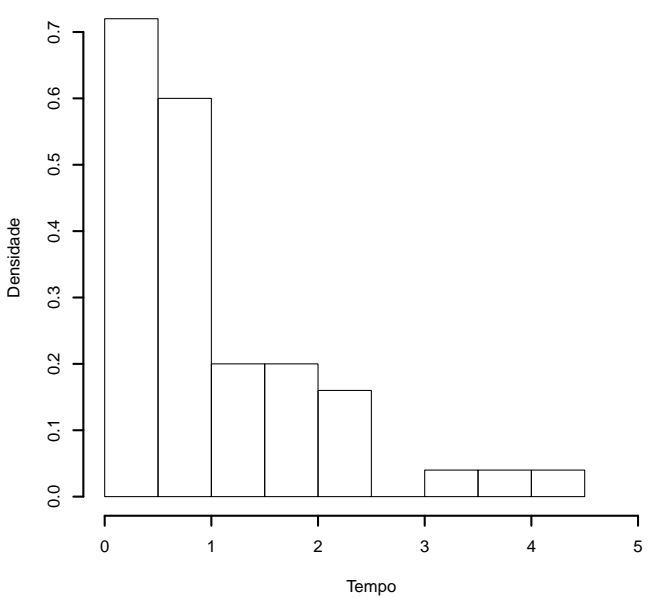

Figura 3.12: (a) Distribuição dos tempos de vida do Estudo 1. (b) Distribuição dos tempos de vida do Estudo 2.

Dado que os tempos de vida seguem distribuição Weibull, a função de verossimilhança para o $j$-ésmio estudo é expressa por:

$$
L_{j}\left(\boldsymbol{t}_{j}, \beta, \eta\right)=\prod_{i=1}^{n_{j}} \frac{\beta}{\eta^{\beta}} t_{i j}^{\beta-1} \exp \left[-\left(\frac{t_{i j}}{\eta}\right)^{\beta}\right] .
$$

Considerando a priori de Jeffreys $\pi(\beta, \eta) \propto \frac{1}{\beta \eta}$ e a função de verossimilhança em (3.34), a distribuição a posteriori de $(\beta, \eta)$ dado $\boldsymbol{t}_{j}$, para o $j$-ésimo estudo, é dada por:

$$
\pi_{j}\left(\beta, \eta \mid \boldsymbol{t}_{j}\right) \propto \frac{1}{\beta \eta} \prod_{i=1}^{n_{j}} \frac{\beta}{\eta^{\beta}} t_{i j}^{\beta-1} \exp \left[-\left(\frac{t_{i j}}{\eta}\right)^{\beta}\right] .
$$

As distribuições a posteriori condicionais de $\beta$ e $\eta$ são dadas, respectivamente, por:

$$
\pi_{j}\left(\beta \mid \boldsymbol{t}_{j}, \eta\right) \propto \frac{\beta^{n_{j}-1}}{\eta^{n_{j} \beta+1}} \prod_{i=1}^{n_{j}} t_{i j}^{\beta-1} \exp \left[-\left(\frac{t_{i} j}{\eta}\right)^{\beta}\right]
$$

$\mathrm{e}$

$$
\pi_{j}\left(\eta \mid \boldsymbol{t}_{j}, \beta\right) \propto \frac{1}{\eta^{n_{j} \beta+1}} \prod_{i=1}^{n_{j}} \exp \left[-\left(\frac{t_{i j}}{\eta}\right)^{\beta}\right] .
$$

Definidas as distribuições a posteriori e condicionais de $\beta$ e $\eta$, o próximo passo será a obtenção das medidas-resumo a posteriori (média, desvio-padrão (DP) e intervalo de $95 \%$ de credibilidade (Int. Cred. 95\%)) para os parâmetros de interesse (Tabela 3.6). Como as distribuições condicionais não são conhecidas, devem ser utilizados métodos de aproximação para a obtenção dessas medidas, como por exemplo, o método de Metropolis-Hastings. As cadeias para $\beta$ e $\eta$ foram geradas com tamanho $m=100.000$, burn in igual a 10.000 e salto de 20. As densidades marginais a posteriori de $\beta$ e $\eta$ encontram-se na Figura 3.14. Como o maior interesse está nas funções de sobrevivência de ambos os estudos, o próximo passo será encontrá-las, de acordo com o Algoritmo 1. A Figura 3.13 contém as curvas de sobrevivência estimadas para ambos os estudos. Observa-se que as curvas estimadas estão muito próximas da verdadeira curva. 
Tabela 3.6: Medidas-resumo a posteriori para os parâmetros do modelo Weibull.

\begin{tabular}{lcccccccc}
\hline & & \multicolumn{3}{c}{$\beta$} & & \multicolumn{3}{c}{$\eta$} \\
\cline { 3 - 5 } \cline { 7 - 9 } & $n$ & Média & DP & Int. Cred. 95\% & & Média & DP & Int. Cred. 95\% \\
\hline Estudo 1 & 50 & 1,73 & 0,19 & {$[1,37 ; 2,13]$} & & 0,97 & 0,09 & {$[0,82 ; 1,15]$} \\
Estudo 2 & 50 & 1,02 & 0,11 & {$[0,79 ; 1,25]$} & & 1,06 & 0,16 & {$[0,77 ; 1,41]$} \\
\hline
\end{tabular}

(a) Estudo 1

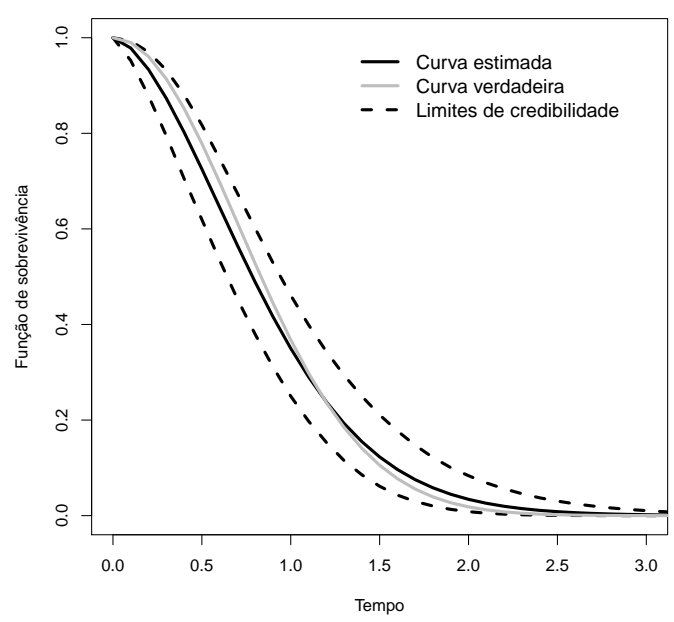

(b) Estudo 2

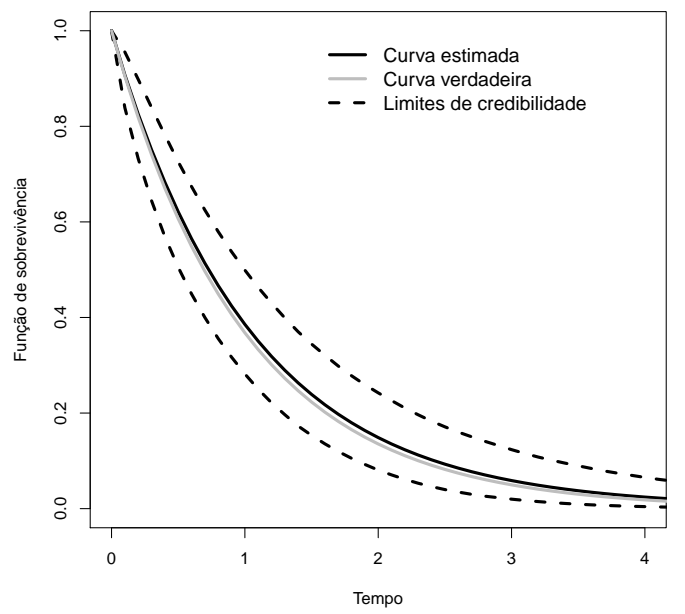

Figura 3.13: Curvas de sobrevivência para o Estudo 1 (a) e para o Estudo 2 (b).

Para a realização de uma metanálise, precisa-se obter uma única curva de sobrevivência que resuma as curvas pertencentes aos outros estudos. Para tal, primeiramente, é necessário obter a distribuição metanalítica de $\beta$ e $\eta$ dada em (3.7).

Considerando $w_{1}=w_{2}=1 / 2$, a distribuição a posteriori metanalítica de $(\beta, \eta), \pi_{M}(\beta, \eta \mid \boldsymbol{t})$, é dada por:

$$
\pi_{M}(\beta, \eta \mid \boldsymbol{t}) \propto \sum_{j=1}^{2} \frac{1}{2} \pi_{j}\left(\beta, \eta \mid \boldsymbol{t}_{j}\right) .
$$

As medidas-resumo (média, desvio-padrão (DP) e intervalo de $95 \%$ de credibilidade (Int. Cred. 95\%)) a posteriori metanalítica para os parâmetros de interesse encontram-se na Tabela 3.7. A Figura 3.14(a) contém a distribuição a posteriori metanalítica marginal de $\beta$ e a Figura 3.14(b), a distribuição a posteriori metanalítica marginal de $\eta$. A Figura 3.15(a) contém a curva de sobrevivência metanalítica e sua banda de $95 \%$ de credibilidade. Já a Figura 3.15(b), contém todas as curvas de sobrevivência para este problema. A probabilidade de sobrevivência pode ser calculada para cada estudo percentente à metanálise e, posteriormente, sua probabilidade de sobrevivência metanálitica. Por exemplo, a probabilidade de um indivíduo sobreviver mais que o tempo 1 no estudo 1 é $S_{1}(t>1)=0,35$; no estudo 2 , é igual a $S_{2}(t>1)=0,39$ e na metanálise, $S_{M}(t>1)=0,37$. As funções de risco para ambos os estudos podem ser calculadas, assim, encontra-se a função de risco metanalítica de forma similar ao Algoritmo 2. Note que o Estudo 2 possui risco constante, e o Estudo 1 e a metanálise apresentam risco crescente.

Tabela 3.7: Medidas-resumo a posteriori para os parâmetros metanalíticos.

\begin{tabular}{cccccccc}
\hline & \multicolumn{3}{c}{$\beta$} & & \multicolumn{3}{c}{$\eta$} \\
\cline { 2 - 4 } \cline { 6 - 8 } \cline { 6 - 8 } & Média & DP & Int. Cred. 95\% & & Média & DP & Int. Cred. 95\% \\
\hline Metanálise & 1,37 & 0,39 & {$[0,83 ; 2,1]$} & & 1,02 & 0,14 & {$[0,79 ; 1,34]$} \\
\hline
\end{tabular}


(a)

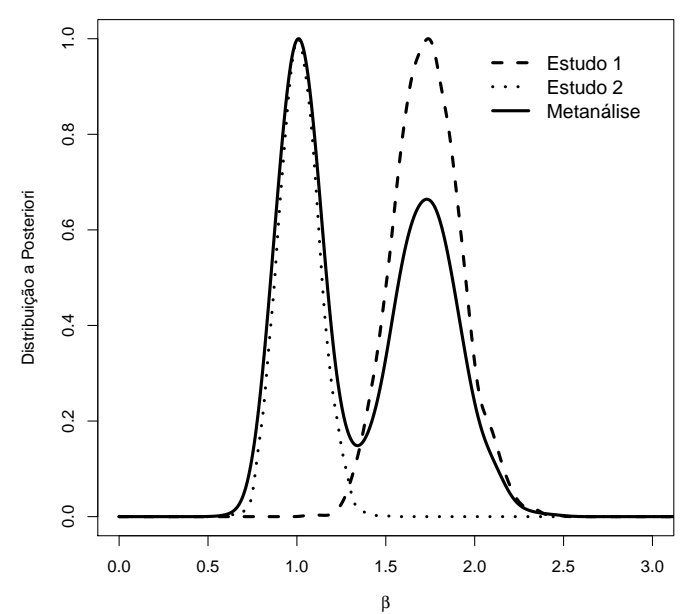

(b)

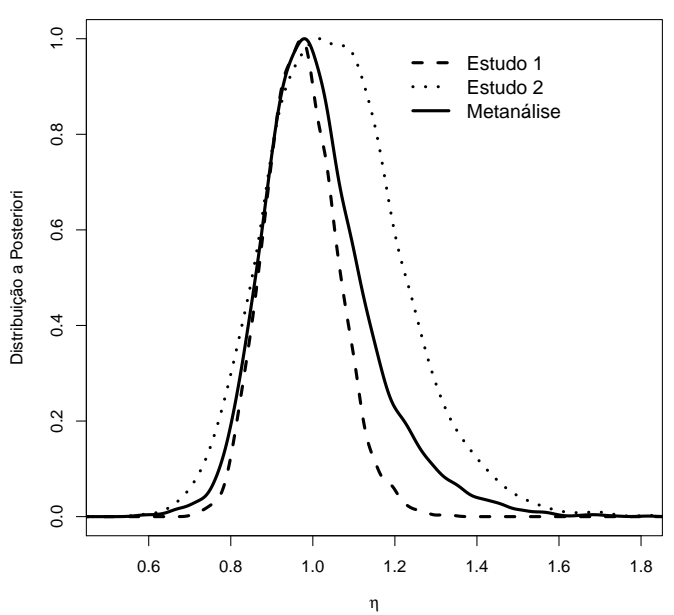

Figura 3.14: (a) Densidades a posteriori estimadas de $\beta$. (b) Densidades a posteriori estimadas de $\eta$.

(a)

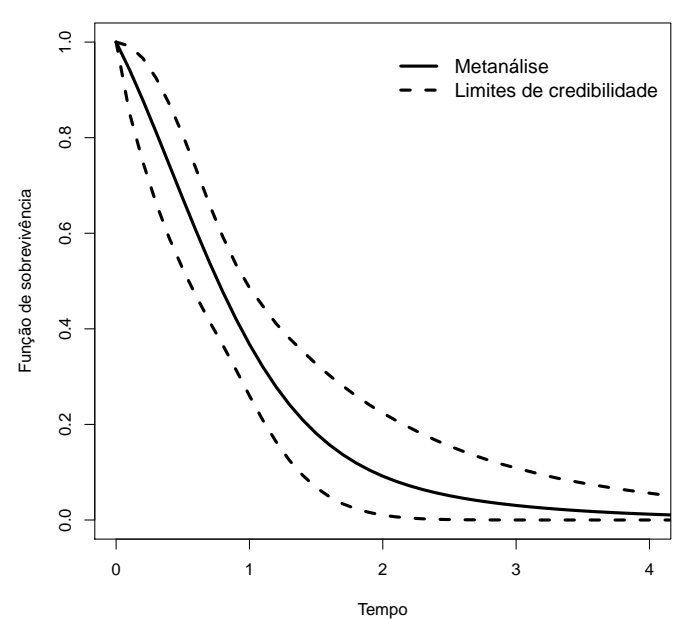

(b)

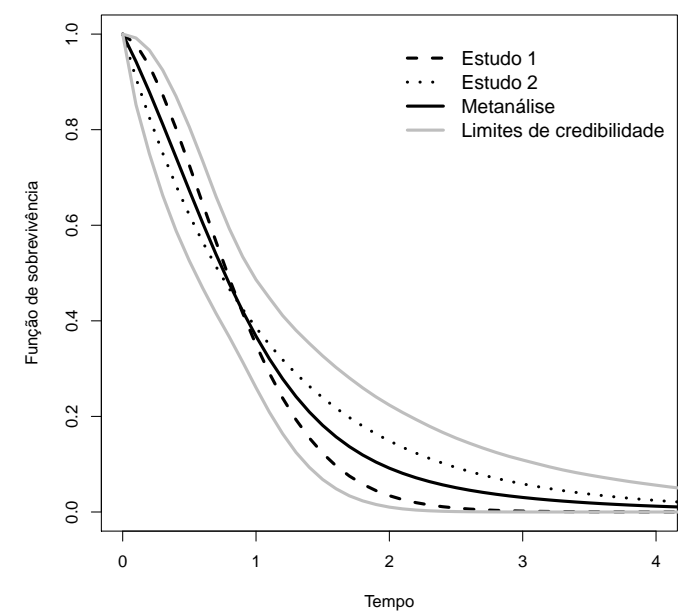

Figura 3.15: Curvas de sobrevivência para o problema.

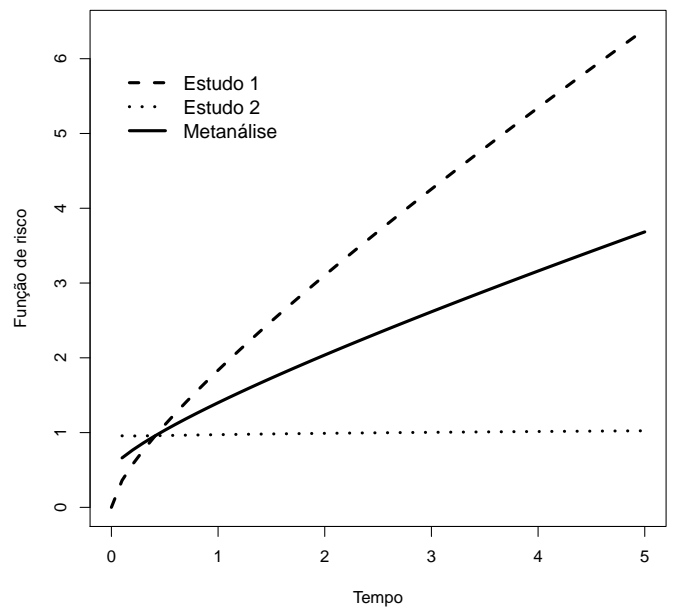

Figura 3.16: Funções de risco para o problema. 


\subsubsection{Exemplo 4: Metanálise com modelos de riscos competitivos}

Muitos estudos em análise de sobrevivência estão centrados em apenas uma causa de morte (falha). Entretanto, causas que competem entre si, para que o evento de interesse ocorra são também comuns. Os estudos que consideram diversas causas de morte (falha) são conhecidos na literatura, como análise de dados de riscos competitivos.

Uma situação comum é que um indivíduo esteja sujeito a diversos fatores de risco de morte e que apenas um desses fatores causará sua morte. Portanto, diz-se que esses fatores estão competindo para a morte desse indivíduo. O modelo para o tempo de vida sujeito a esses fatores é conhecido como modelo de fatores de risco competitivos ou, por apenas, modelo de riscos competitivos. Cada indivíduo está exposto a $\mathrm{C}$ fatores de risco independentes, e somente um causará a morte desse indivíduo. O fator é conhecido como a causa da morte.

Suponha que $X_{i 1}, X_{i 2}, \ldots, X_{i \mathrm{C}}$ sejam variáveis aleatórias independentes, contínuas e positivas denominadas tempos de vida para um determinado indivíduo $i(i=1, \ldots, n)$. Ou seja, $X_{i \ell}$ representa o tempo de vida do $i$-ésimo indivíduo sob o $\ell$-ésimo fator de risco de morte. Contudo, todos os $\mathrm{C}$ fatores agem simultaneamente, e como somente um irá causar a morte do indivíduo, será adotada outra variável aleátoria $T_{i}=\min \left\{X_{i 1}, X_{i 2}, \ldots, X_{i c}\right\}$. Além disso, observa-se uma variável indicadora $\delta_{i \ell}$, que representa a causa da falha do $i$-ésimo indivíduo sob o $\ell$-ésimo fator de risco, ou seja,

$$
\delta_{i \ell}=\left\{\begin{array}{l}
1, \text { se o indivíduo } i \text { morreu por } \ell, \text { ou seja, } T_{i}=X_{i \ell} \\
0, \text { se o indivíduo } i \text { não morreu por } \ell, \text { ou seja, } T_{i} \neq X_{i \ell}
\end{array} .\right.
$$

Considerando uma amostra de $n$ observações nas condições anteriores, os dados estão dispostos da seguinte forma: $\left(T_{1}, \delta_{11}, \delta_{12}, \ldots, \delta_{1 \mathrm{C}}\right), \ldots,\left(T_{n}, \delta_{n 1}, \delta_{n 2}, \ldots, \delta_{n \mathrm{C}}\right)$. Na prática, tais dados são com frequência censurados à direita por alguma causa independente dos C riscos. Por exemplo, a censura poderia ser por causa do tempo limite do experimento. Se o $i$-ésimo indivíduo é não censurado, ou seja, morreu por $\ell$, observa-se o tempo de morte $T_{i}$ e a indicadora de morte/ falha $\delta_{i \ell}=1$. Por outro lado, se o $i$-ésimo indivíduo é censurado à direita, sabe-se apenas que $X_{i \ell}>T_{i}$. Então, a função de verossimilhança (Lindqvist [2006]) é dada por:

$$
\begin{aligned}
L\left(\boldsymbol{t}, \theta_{1}, \ldots, \theta_{\mathrm{C}}\right) & =\prod_{\ell=1}^{\mathrm{C}} \prod_{i=1}^{n} f\left(t_{i} \mid \theta_{\ell}\right)^{\delta_{i \ell}} S\left(t_{i} \mid \theta_{\ell}\right)^{1-\delta_{i \ell}} \\
& =\prod_{\ell=1}^{\mathrm{C}} L_{\ell}\left(\boldsymbol{t}, \theta_{\ell}\right) .
\end{aligned}
$$

Pode-se escrever $L\left(\boldsymbol{t}, \theta_{1}, \ldots, \theta_{\mathrm{C}}\right)$ como o produto de verossimilhanças $L_{\ell}\left(\boldsymbol{t}, \theta_{\ell}\right)$, em que $L_{\ell}\left(\boldsymbol{t}, \theta_{\ell}\right)=$ $\prod_{i=1}^{n} f_{\ell}\left(t_{i}\right)^{\delta_{i \ell}} S_{\ell}\left(t_{i}\right)^{1-\delta_{i \ell}}$ tem a forma da função de verossimilhança de uma amostra censurada (maiores detalhes ver Rodrigues et al. [2008]) com a $\ell$-ésima causa de morte em que todas as observações, onde $T_{i} \neq X_{i \ell}$, são tratadas como censura.

Note que, quando se analisa um determinado fator $\ell$, o modelo de fatores de risco competitivos considera como morte/ falha os tempos em que as mortes ocorreram pelo fator $\ell$ e considera como censura, além dos tempos que não apresentaram mortes, os tempos de falha provocados por fatores diferentes de $\ell$.

Considere uma amostra de $n$ tempos $t_{i}(i=1,2, \ldots, n)$ com os respectivos rótulos $\delta_{i \ell}$. Suponha que para uma população que siga o modelo Weibull (3.30), existam C fatores de morte independentes. As funções densidade e de sobrevivência sob o modelo Weibull na aplicação de riscos competitivos, para o indivíduo $i$, são dadas, respectivamente, por

$$
f\left(t_{i}, \beta_{\ell}, \eta_{\ell}\right)=\frac{\beta_{\ell}}{\eta_{\ell}^{\beta_{\ell}}} t_{i}^{\beta_{\ell}-1} \exp \left[-\left(\frac{t_{i}}{\eta_{\ell}}\right)^{\beta_{\ell}}\right], \ell=1,2, \ldots, \mathrm{C}
$$


$\mathrm{e}$

$$
S\left(t_{i}, \beta_{\ell}, \eta_{\ell}\right)=\exp \left[-\left(\frac{t_{i}}{\eta_{\ell}}\right)^{\beta_{\ell}}\right] .
$$

Substituindo (3.41) e (3.42) em (3.39), a função de verossimilhança sob o modelo Weibull assume a forma

$$
L(\boldsymbol{t}, \boldsymbol{\beta}, \boldsymbol{\eta})=\prod_{i=1}^{n} \prod_{\ell=1}^{\mathrm{C}}\left(\frac{\beta_{\ell}}{\eta_{\ell}^{\beta_{\ell}}} t_{i}^{\beta_{\ell}-1}\right)^{\delta_{i \ell}} \exp \left[-\left(\frac{t_{i}}{\eta_{\ell}}\right)^{\beta_{\ell}}\right] .
$$

Considerando a priori de Jeffreys, $\pi(\boldsymbol{\eta}, \boldsymbol{\beta}) \propto \prod_{\ell=1}^{\mathrm{C}} \frac{1}{\eta_{\ell} \beta_{\ell}}$, e a função de verossimilhança em (3.43), a distribuição a posteriori de $(\boldsymbol{\beta}, \boldsymbol{\eta})$ é dada por

$$
\pi(\boldsymbol{\beta}, \boldsymbol{\eta} \mid \boldsymbol{t}) \propto \prod_{i=1}^{n} \prod_{\ell=1}^{\mathrm{C}}\left(\left(\frac{\beta_{\ell}}{\eta_{\ell}^{\beta_{\ell}}} t_{i}^{\beta_{\ell}-1}\right)^{\delta_{i \ell}} \exp \left[-\left(\frac{t_{i}}{\eta_{\ell}}\right)^{\beta_{\ell}}\right] \frac{1}{\eta_{\ell} \beta_{\ell}}\right) .
$$

Portanto, para uma metanálise com dados de riscos competitivos, considerando $j$ estudos, a distribuição metanalítica de $(\boldsymbol{\beta}, \boldsymbol{\eta})$ é dada por:

$$
\pi_{M}(\boldsymbol{\beta}, \boldsymbol{\eta} \mid \boldsymbol{t})=\sum_{j=1}^{J} \omega_{j} \pi_{j}\left(\boldsymbol{\beta}, \boldsymbol{\eta} \mid \boldsymbol{t}_{j}\right)
$$

em que $\omega_{j}=\frac{n_{j}}{\sum_{j=1}^{J} n_{j}}$ é o peso do $j$-ésimo estudo e $\pi_{j}\left(\boldsymbol{\beta}, \boldsymbol{\eta} \mid \boldsymbol{t}_{j}\right)$ é a distribuição a posteriori conjunta de $(\boldsymbol{\beta}, \boldsymbol{\eta})$ para o $j$-ésimo estudo como em (3.44).

Considere uma metanálise composta de três estudos $(J=3)$ relacionados ao tempo de vida de pacientes com câncer expostos a três causas de morte $(\mathrm{C}=3)$ : Fator 1: o próprio câncer; Fator 2: algum tipo de infecção ou Fator 3: fatores externos. Portanto, são gerados três conjuntos de dados com tamanhos amostrais iguais a $n_{1}=100, n_{2}=120, n_{3}=90$, conforme o modelo Weibull (3.41). Os parâmetros utilizados na geração dos conjuntos de dados encontram-se na Tabela 3.8. Os dados estão dispostos na Tabela A.4, e as medidas-resumo dos tempos de vida (mínimo (Min), primeiro quartil (Q1), mediana (Med), média, terceiro quartil (Q3), máximo (Max), desvio-padrão (DP) e $\left.\Delta_{\ell}=\sum_{i=1}^{n_{j}} \delta_{i \ell}, j=\ell=1,2,3\right)$, para todos os estudos, encontram-se na Tabela 3.9 .

Tabela 3.8: Parâmetros do modelo Weibull.

\begin{tabular}{lcccccc}
\hline & $\beta_{1}$ & $\eta_{1}$ & $\beta_{2}$ & $\eta_{2}$ & $\beta_{3}$ & $\eta_{3}$ \\
\hline Estudo 1 & 2 & 10 & 3 & 10 & 4 & 10 \\
Estudo 2 & 2.5 & 10 & 3.5 & 10 & 5 & 10 \\
Estudo 3 & 3 & 10 & 3 & 10 & 4 & 10 \\
\hline
\end{tabular}

Tabela 3.9: Medidas-resumo do tempo de sobrevivência independente do fator de risco em cada estudo pertencente à metanálise.

\begin{tabular}{lccccccccccc}
\hline & $\mathrm{n}$ & Min & Q1 & Med & Média & Q3 & Max & DP & $\Delta_{1}$ & $\Delta_{2}$ & $\Delta_{3}$ \\
\hline Estudo 1 & 100 & 0,88 & 4,05 & 5,65 & 5,70 & 7,33 & 11,15 & 2,31 & 44 & 32 & 24 \\
Estudo 2 & 120 & 1,81 & 5,23 & 6,61 & 6,75 & 8,10 & 12,05 & 2,03 & 47 & 39 & 34 \\
Estudo 3 & 90 & 0,93 & 5,55 & 6,84 & 6,62 & 8,15 & 10,37 & 2,13 & 32 & 31 & 27 \\
\hline
\end{tabular}


Para se estimar os parâmetros em questão e as curvas de sobrevivência para cada estudo, assim como as medidas metanalíticas, foi obtida uma amostra das distribuições a posteriori marginais de $\beta_{\ell}$ e $\eta_{\ell}(\ell=1,2,3)$ para cada estudo, utilizando o algoritmo de Metropolis-Hastings. As medidasresumo (média, desvio-padrão (DP) e intervalo de 95\% de credibilidade (Int. Cred. 95\%)) das amostras a posteriori de todas as cadeias simuladas, adotando aquecimento (burn in) de 10.000, com tamanho inicial de 100.000 e salto igual a 10, encontram-se na Tabela 3.10, Tabela 3.11 e Tabela 3.12 .

Tabela 3.10: Medidas-resumo a posteriori para os parâmetros relacionados ao fator de risco 1.

\begin{tabular}{lcccccccc}
\hline & & \multicolumn{3}{c}{$\beta_{1}$} & & \multicolumn{3}{c}{$\eta_{1}$} \\
\cline { 3 - 5 } \cline { 7 - 9 } & $n$ & Média & DP & Int. Cred. 95\% & & Média & DP & Int. Cred. 95\% \\
\hline Estudo 1 & 100 & 1,97 & 0,12 & {$[1,74 ; 2,21]$} & & 9,94 & 0,52 & {$[8,67 ; 10,71]$} \\
Estudo 2 & 120 & 2,56 & 0,14 & {$[2,29 ; 2,84]$} & & 10,12 & 0,34 & {$[9,47 ; 10,80]$} \\
Estudo 3 & 90 & 2,99 & 0,07 & {$[2,86 ; 3,13]$} & & 10,01 & 0,19 & {$[9,64 ; 10,40]$} \\
\hline
\end{tabular}

Tabela 3.11: Medidas-resumo a posteriori para os parâmetros relacionados ao fator de risco 2.

\begin{tabular}{lcccccccc}
\hline & & \multicolumn{4}{c}{$\beta_{2}$} & & \multicolumn{3}{c}{$\eta_{2}$} \\
\cline { 3 - 5 } \cline { 7 - 9 } & $n$ & Média & DP & Int. Cred. 95\% & & Média & DP & Int. Cred. 95\% \\
\hline Estudo 1 & 100 & 2,99 & 0,17 & {$[2,66 ; 3,35]$} & & 9,75 & 0,42 & {$[8,98 ; 10,62]$} \\
Estudo 2 & 120 & 3,50 & 0,18 & {$[3,15 ; 3,88]$} & & 10,10 & 0,28 & {$[9,56 ; 10,65]$} \\
Estudo 3 & 90 & 3,00 & 0,07 & {$[2,87 ; 3,14]$} & & 10,03 & 0,19 & {$[9,65 ; 10,40]$} \\
\hline
\end{tabular}

Tabela 3.12: Medidas-resumo a posteriori para os parâmetros relacionados ao fator de risco 3 .

\begin{tabular}{lcccccccc}
\hline & & \multicolumn{4}{c}{$\beta_{3}$} & & \multicolumn{3}{c}{$\eta_{3}$} \\
\cline { 3 - 5 } \cline { 7 - 9 } & $n$ & Média & DP & Int. Cred. 95\% & & Média & DP & Int. Cred. 95\% \\
\hline Estudo 1 & 100 & 3,97 & 0,27 & {$[3,45 ; 4,51]$} & & 9,90 & 0,34 & {$[9,26 ; 10,60]$} \\
Estudo 2 & 120 & 5,13 & 0,31 & {$[4,53 ; 5,77]$} & & 10,02 & 0,24 & {$[9,56 ; 10,51]$} \\
Estudo 3 & 90 & 4,07 & 0,14 & {$[3,80 ; 4,34]$} & & 9,99 & 0,18 & {$[9,63 ; 10,36]$} \\
\hline
\end{tabular}

Ao analisar os dados das Tabela 3.10, a Tabela 3.11 e a Tabela 3.12, observa-se que as estimativas dos parâmetros de interesse estão próximas do verdadeiro valor de cada parâmetro e os intervalos de $95 \%$ de credibilidade contêm o verdadeiro valor de cada parâmetro utilizado na simulação. As funções densidade a posteriori marginal estimadas dos parâmetros dos fatores de risco 1,2 e 3 , assim como o metanalítico, encontram-se na Figura 3.17. Posteriormente, ao encontrar as distribuições a posteriori dos parâmetros de interesse e seus respectivos valores esperados, para cada estudo pertencente à metanálise, o próximo passo será a estimação das curvas de sobrevivência via MH por meio do Algoritmo 1. A Figura 3.18 contém as curvas de sobrevivência estimadas para cada fator de risco e para o indivíduo utilizando os dados do Estudo 1. O mesmo ocorre para o Estudo 2, Figura 3.19, e para o Estudo 3, Figura 3.20. Verifica-se que o ajuste do modelo Weibull é adequado aos dados em questão, indicando o esperado.

Utilizando (3.45), calcula-se a curva de sobrevivência metanalítica reportada no Algoritmo 2. As Figura 3.21(a), Figura 3.22(a) e Figura 3.23(a) contêm as curvas de sobrevivência metanalítica dos Fatores 1, 2 e 3, respectivamente, com suas bandas de $95 \%$ de credibilidade. Já as Figuras 3.21 (b), 3.22(b) e 3.23(b) contêm todas as curvas de sobrevivência para esses fatores. A função de sobrevivência metanalítica estimada para o indivíduo encontra-se na Figura 3.24. 
(a) Fator 1

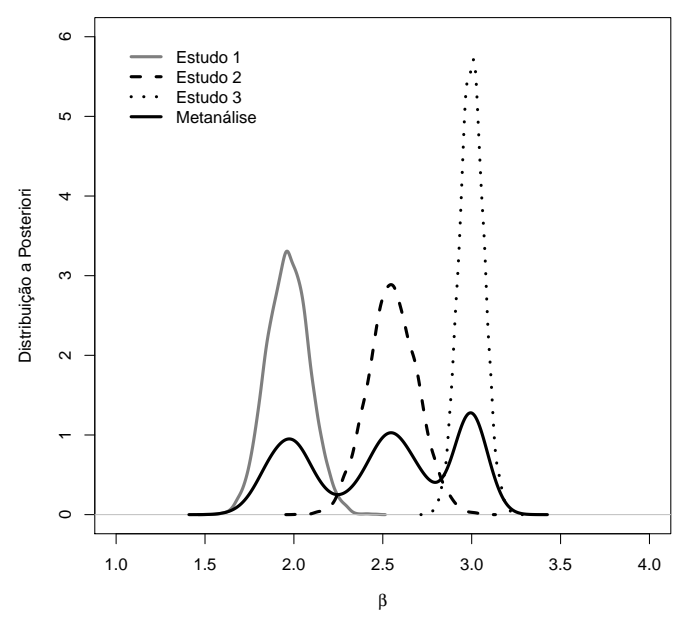

(c) Fator 2

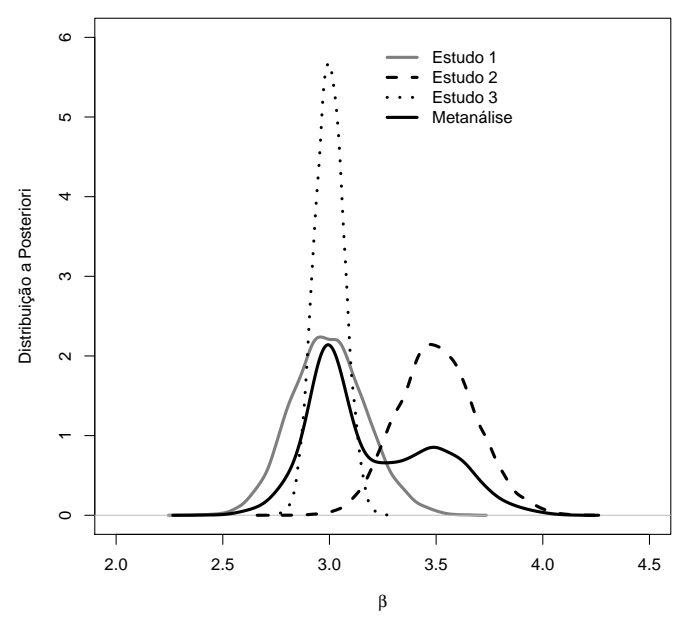

(e) Fator 3

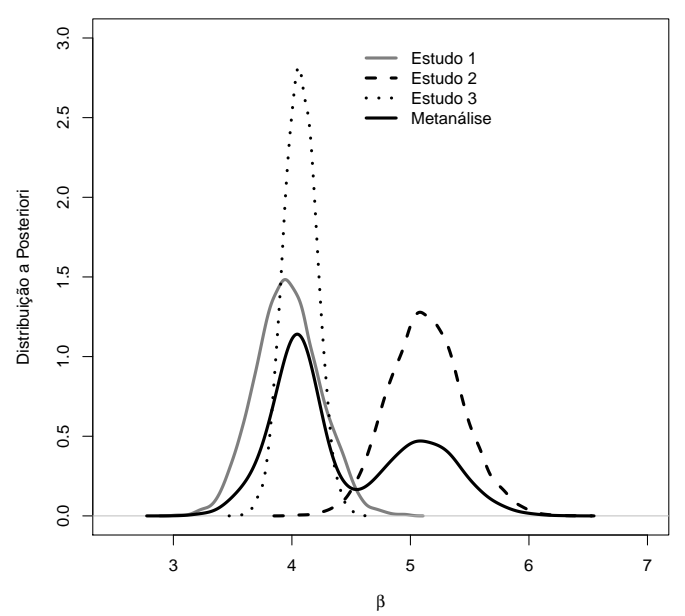

(b)

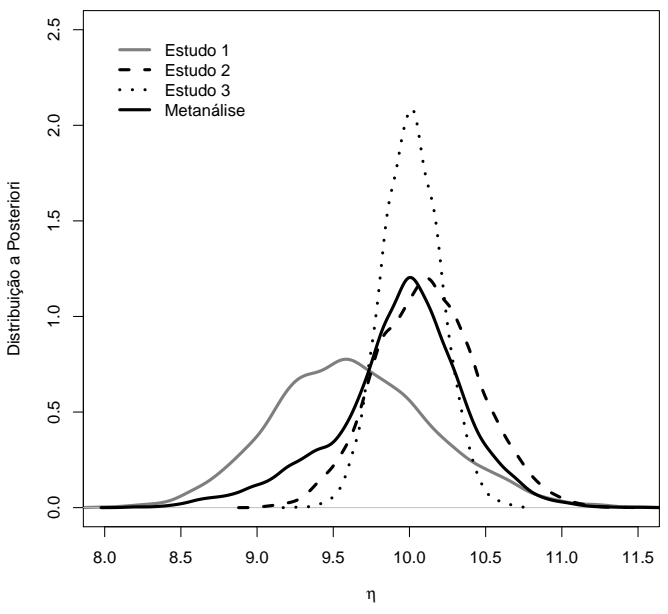

(d)

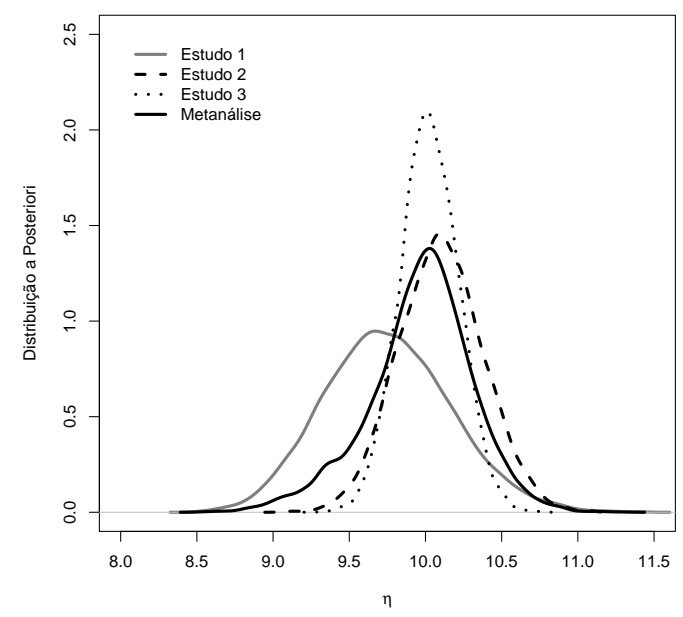

(f)

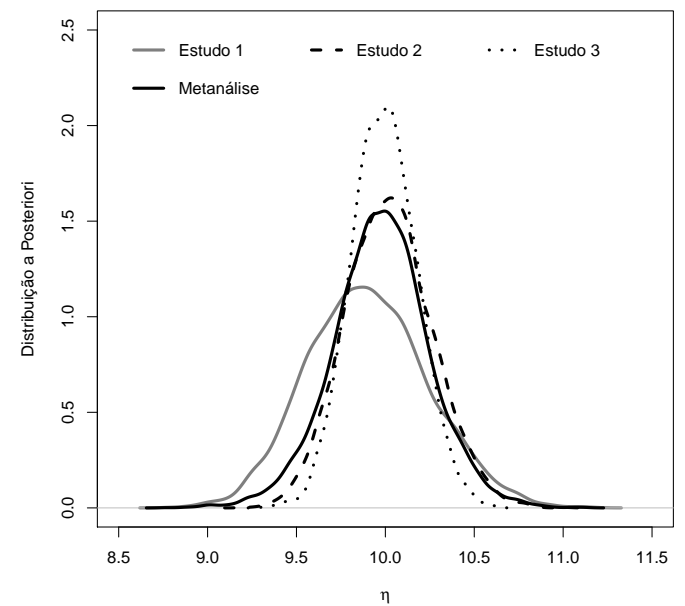

Figura 3.17: Distribuições a posteriori estimadas dos parâmetros do fator de risco $1((a)$ e (b)), do fator de risco 2 ((c) e (d)) e do fator de risco 3 ((e) e (f)). 

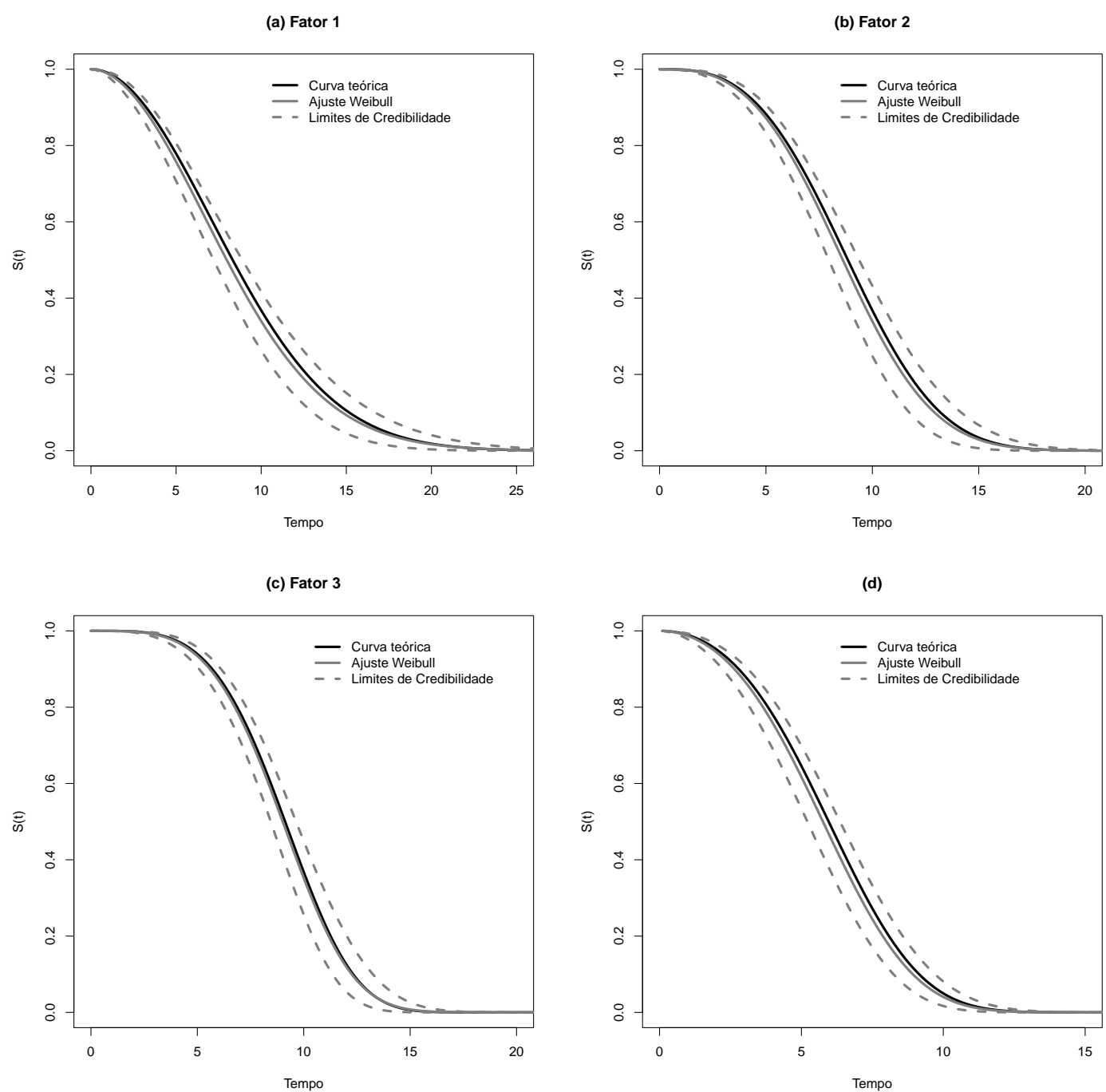

Figura 3.18: Ajustes referentes ao Estudo 1. (a) Ajuste da função de sobrevivência dos fatores de risco 1 (a), 2 (b), 3 (c) e do indivíduo (d). 

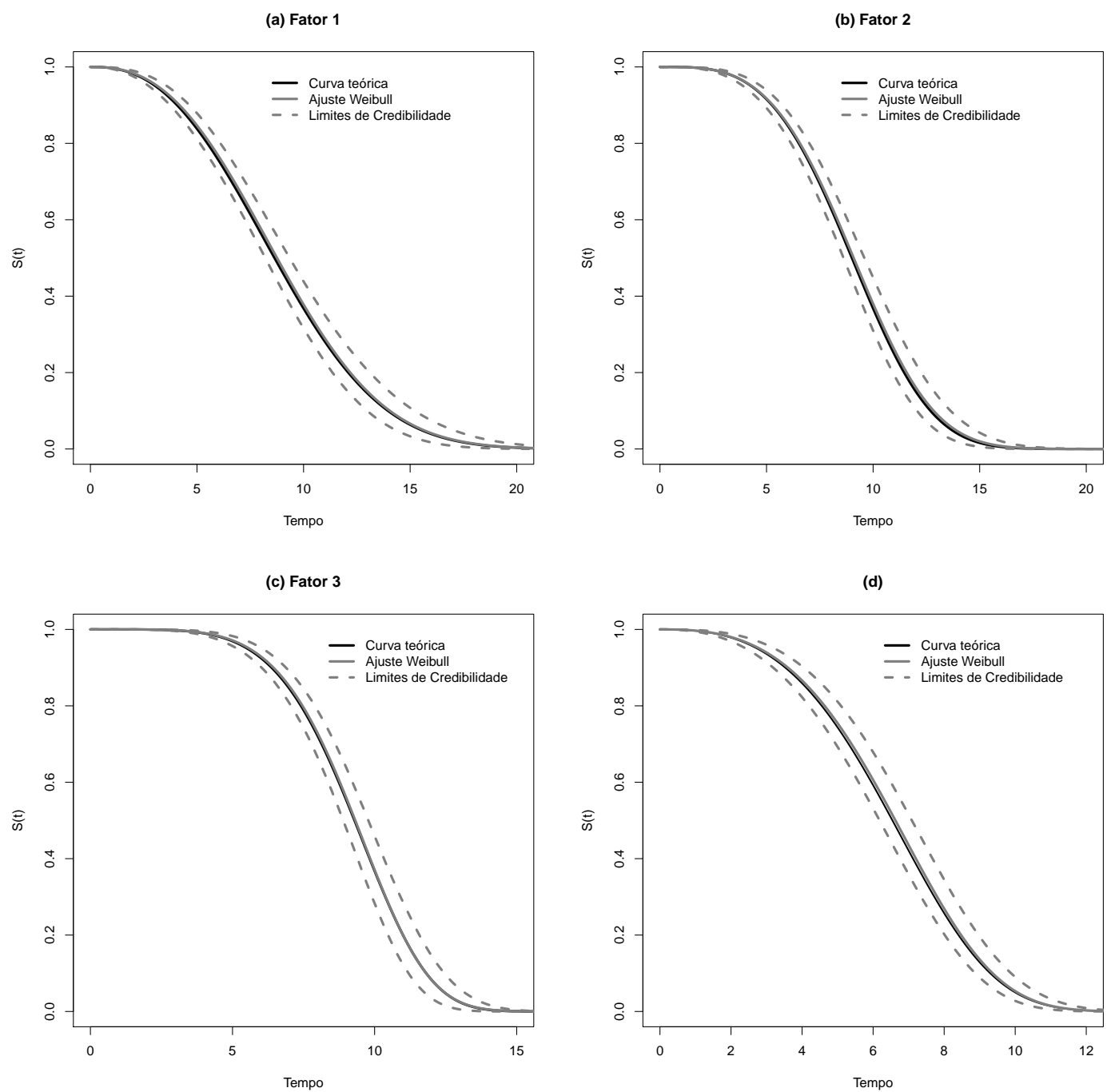

Figura 3.19: Ajustes referentes ao Estudo 2. (a) Ajuste da função de sobrevivência dos fatores de risco 1 (a), 2 (b), 3 (c) e do indivíduo (d). 

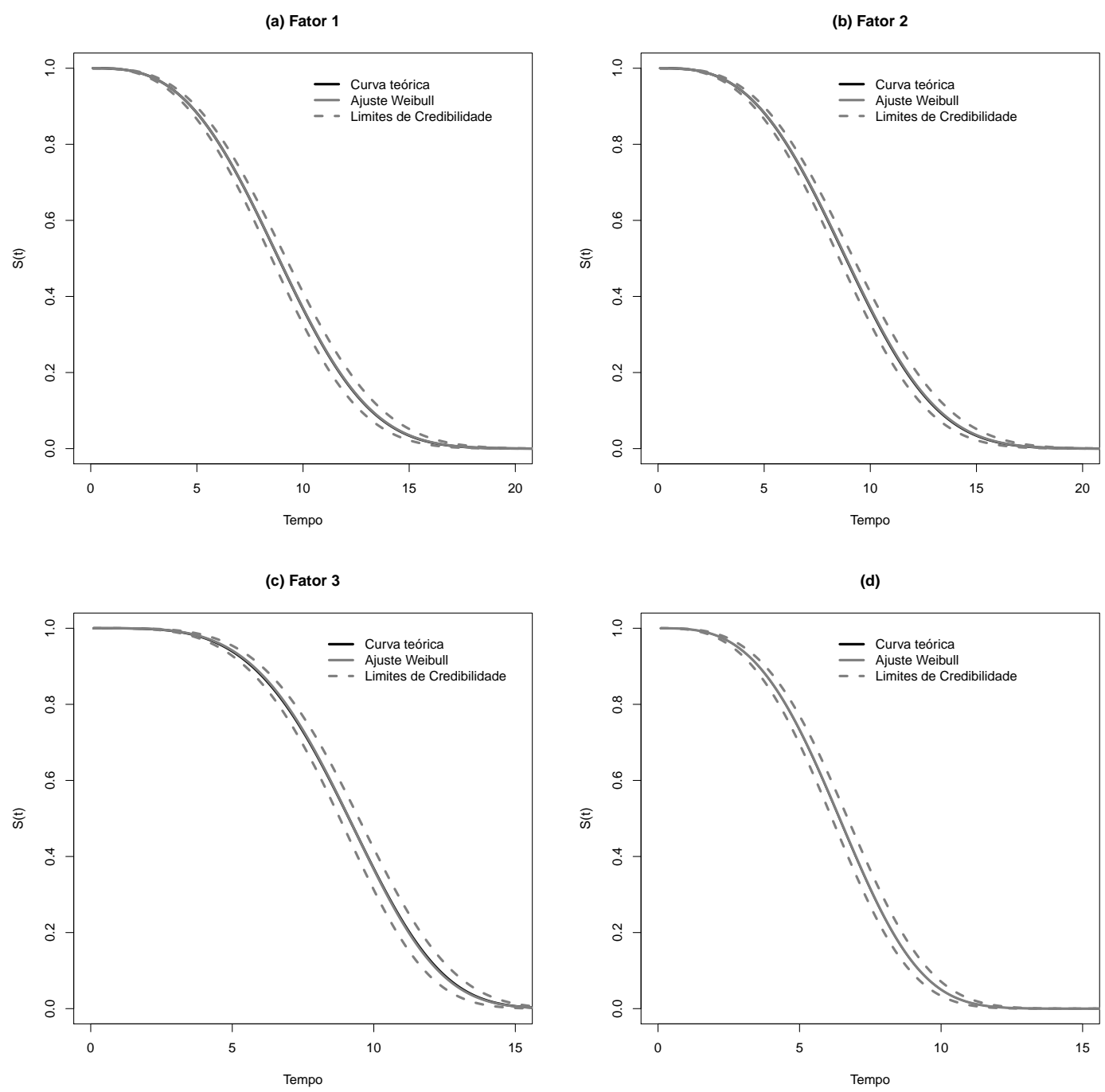

Figura 3.20: Ajustes referentes ao Estudo 3. (a) Ajuste da função de sobrevivência dos fatores de risco 1 (a), 2 (b), 3 (c) e do indivíduo (d).

(a) Fator 1

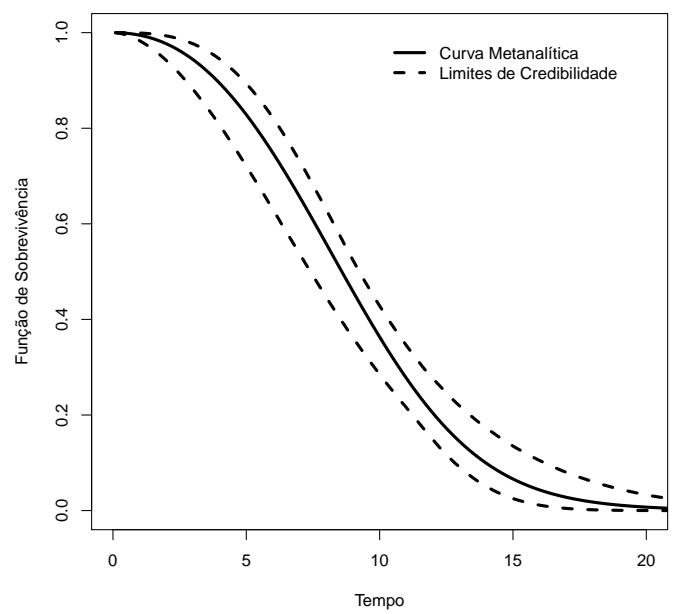

(b)

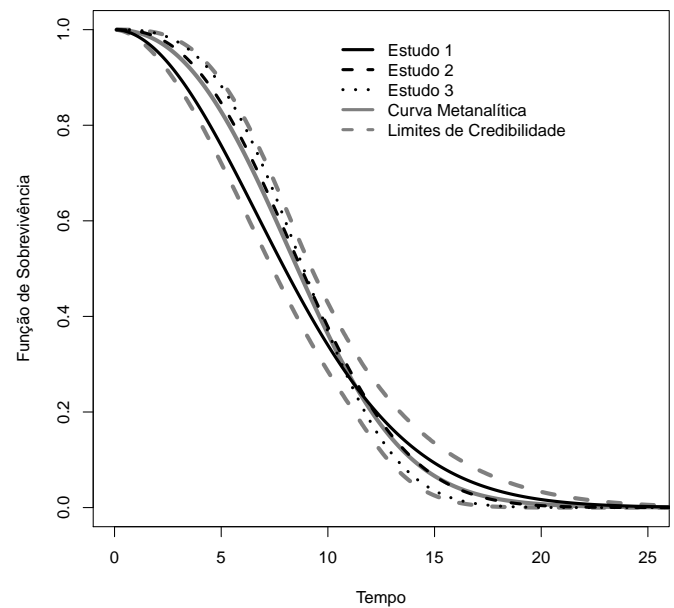

Figura 3.21: (a) Curva de sobrevivência metanalítica para o fator de risco 1 e banda de credibilidade de 95\%. (b) Curvas de sobrevivência relacionadas ao Fator 1. 
(a) Fator 2

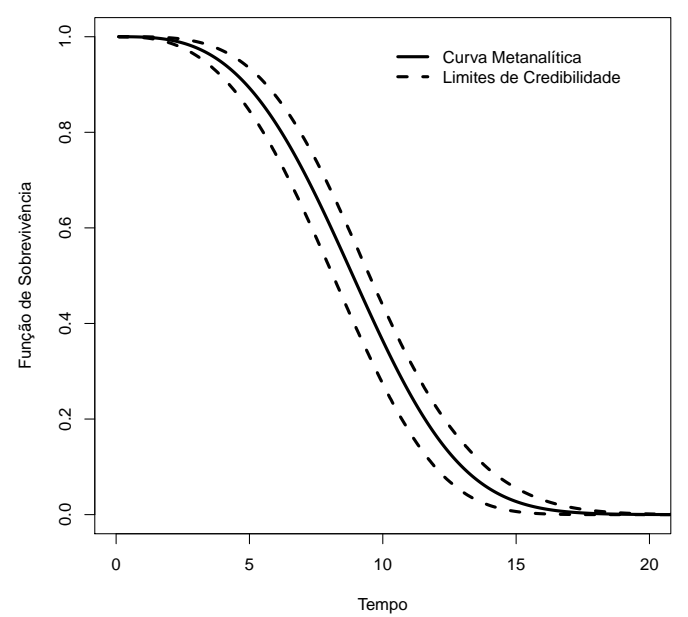

(b)

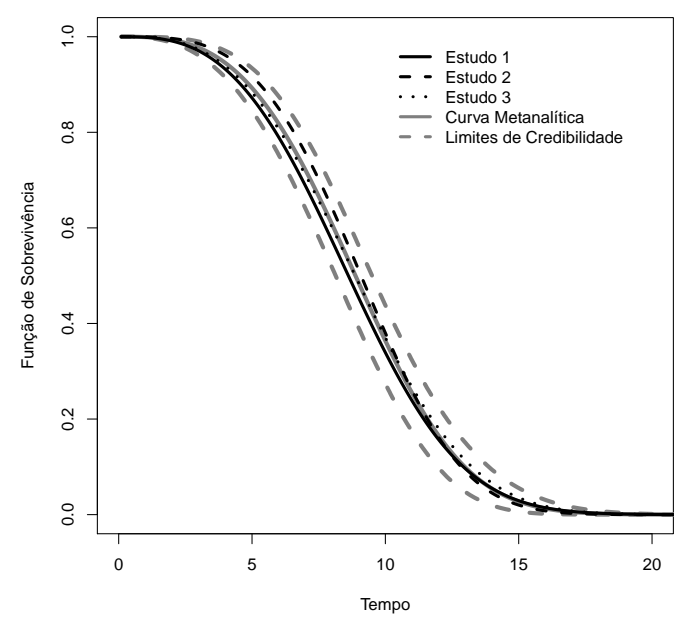

Figura 3.22: (a) Curva de sobrevivência metanalítica para o fator de risco 2 e banda de credibilidade de 95\%. (b) Curvas de sobrevivência relacionadas ao Fator 2.

(a) Fator 3

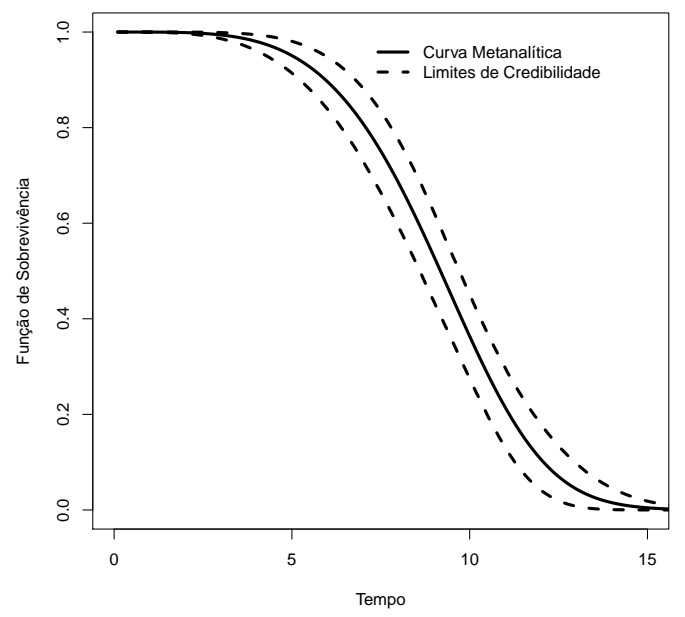

(b)

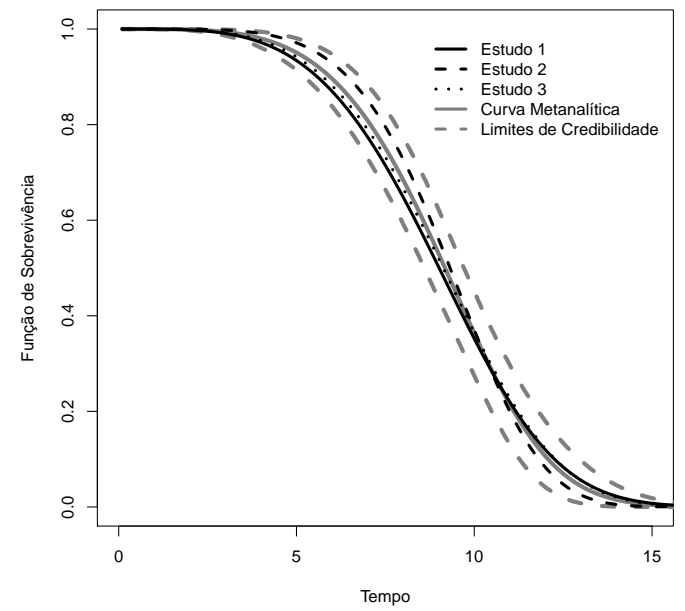

Figura 3.23: (a) Curva de sobrevivência metanalítica para o fator de risco 3 e banda de credibilidade de $95 \%$. (b) Curvas de sobrevivência relacionadas ao Fator 3. 
(a)

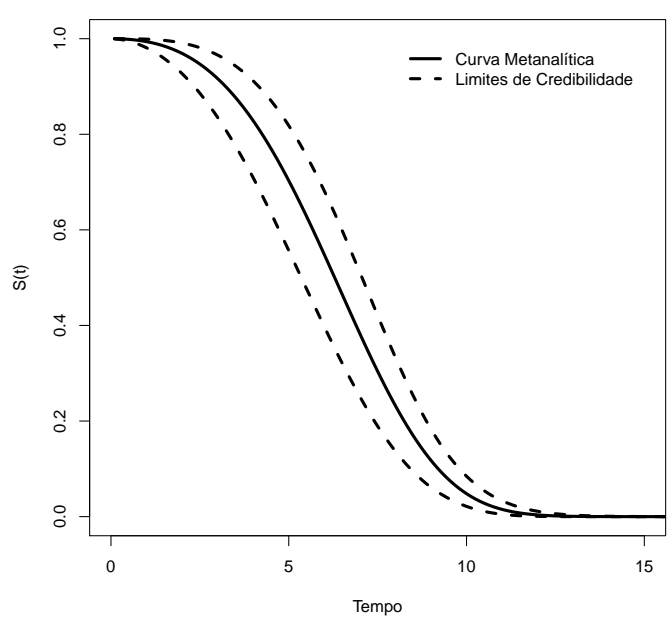

(b)

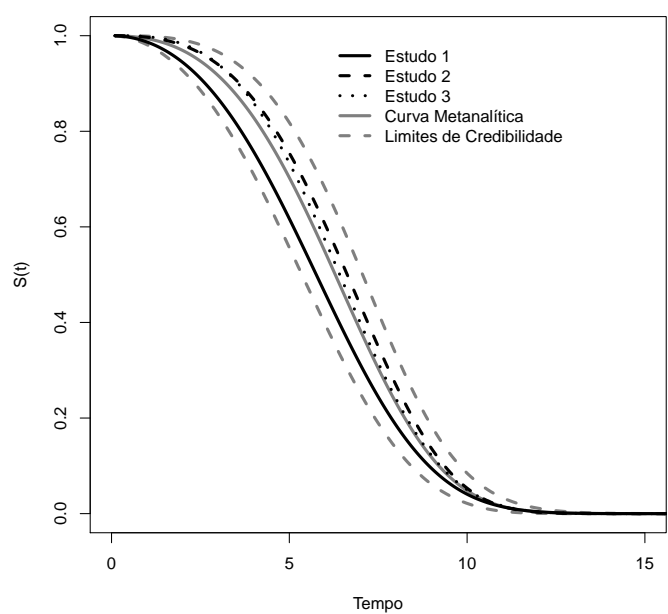

Figura 3.24: (a) Curva de sobrevivência metanalitica do indivíduo e banda de credibilidade de 95\%. (b) Curvas de sobrevivência do indivíduo.

A probabilidade de sobrevivência pode ser calculada dado um determinado fator de risco para cada estudo pertencente à metanálise e, posteriormente, a probabilidade de sobrevivência metanalítica de cada fator de risco. Por exemplo, a probabilidade de um indivíduo, exposto ao fator de risco 1 (câncer), sobreviver mais que o tempo 5 no estudo 1 é de $S_{11}(t>5)=0,76$; no estudo 2 , é igual a $S_{12}\left(t_{2}>5\right)=0,85$ e no estudo $3, S_{13}\left(t_{1}>5\right)=0,88$. Já a probabilidade metanalítica de um indivíduo exposto ao fator de risco 1 sobreviver mais que o tempo 5 é igual a 0,83 . A probabilidade de um indivíduo sobreviver, independente do fator de risco, mais que 5 é igual 0,63 no estudo 1. Considerando o tempo $t=5$, calcula-se a probabilidade de sobrevivência para indivíduos expostos aos fatores de risco em todos os estudos e na metanálise, Tabela 3.13.

Tabela 3.13: Calculo da probabilidade de um indivíduo sobreviver mais que o tempo 5.

\begin{tabular}{ccccc}
\hline & Estudo 1 & Estudo 2 & Estudo 3 & Metanálise \\
\hline Fator 1 & 0,76 & 0,85 & 0,88 & 0,83 \\
Fator 2 & 0,87 & 0,92 & 0,88 & 0,89 \\
Fator 3 & 0,93 & 0,97 & 0,94 & 0,95 \\
Indivíduo & 0,62 & 0,75 & 0,73 & 0,70 \\
\hline
\end{tabular}

\subsubsection{Exemplo 5: Modelos de regressão em análise de sobrevivência}

Considere uma metanálise composta de dois estudos reais, que se referem ao uso de antipsicóticos e ao tempo até a próxima re-hospitalização de pacientes com esquizofrenia. Os dados foram fornecidos pelos pesquisadores Herceg et al. [2008] e Werneck et al. [2011]. De acordo com Werneck et al. [2011], a re-hospitalização é uma medida de desfecho reconhecida e utilizada em estudos para acessar a prevenção de recaída, que é considerada um dos principais indicadores de efetividade de um antipsicótico. Dentre as variáveis coletadas, foram analisadas as covariáveis: idade, gênero (masculino ou feminino), tipo de antipsicótico (típico ou atípico), tempo de re-hospitalização e o indicador de internação (indicador de censura) dos pacientes com esquizofrenia.

Primeiramente, foi realizada uma análise descritiva dos dados com o objetivo de traçar o perfil das amostras de ambos os estudos. Para as variáveis contínuas, idade e tempo de re-hospitalização, foram calculados: mínimo, máximo, mediana, média, erro-padrão (EP) e desvio-padrão (DP) (Tabela 3.14). A idade média dos pacientes com esquizofrenia pertencentes ao Estudo 1 é de 38, 7 anos e, para os pacientes do Estudo 2 é de 41,8 anos. O tempo de re-hospitalização médio dos pacientes esquizofrênicos para os estudos são, respectivamente, iguais a 1,6 e 1,3 anos. O desvio-padrão do 
tempo de re-hospitalização são relativamente altos em ambos os estudos, 1,22 e 0,98 anos. A distribuição do tempo de re-hospitzalização pode ser vista através do Box Plot, Figura 3.25. Para as variáveis categóricas, gênero, tipo de antipsicótico e indicador de internação, calculamos a frequência e o percentual (Tabela 3.15). O percentual de pacientes do gênero masculino é de 55, $8 \%$ no Estudo 1 e de $65,1 \%$, no Estudo 2. No Estudo 1, o percentual de uso do antipsicótico típico é de 28,9\% e, já no Estudo 2, é de $58 \%$. O percentual de re-internação nos estudos 1 e 2 é, respectivamente, $19,8 \%$ e $49,5 \%$.

Tabela 3.14: Medidas-resumo das variáveis Tempo de re-hospitalização (anos) e Idade.

\begin{tabular}{lcccccccc}
\hline & Variáveis & $\mathrm{N}$ & Mínimo & Máximo & Mediana & Média & $\mathrm{EP}$ & $\mathrm{DP}$ \\
\hline Estudo 1 & Tempo & 242 & 0,008 & 3 & 1,295 & 1,559 & 0,078 & 1,221 \\
& Idade & 242 & 21 & 77 & 37 & 38,70 & 0,66 & 10,20 \\
\hline Estudo 2 & Tempo & 398 & 0,003 & 2,975 & 1,123 & 1,332 & 0,049 & 0,983 \\
& Idade & 398 & 21 & 65 & 42 & 41,82 & 0,50 & 10,06 \\
\hline
\end{tabular}

Tabela 3.15: Frequência e percentual das variáveis Gênero, Tipo de antipsicótico e Censura.

\begin{tabular}{ccccc}
\hline & \multicolumn{2}{c}{ Estudo 1 } & \multicolumn{2}{c}{ Estudo 2 } \\
\hline Gênero & Frequência & Percentual $(\%)$ & Frequência & Percentual (\%) \\
Masculino & 135 & 55,8 & 259 & 65,1 \\
Feminino & 107 & 44,2 & 139 & 34,9 \\
Total & 242 & 100,0 & 398 & 100,0 \\
& & & & \\
Tipo de antipsicótico & & & & 58,0 \\
Típico & 70 & 28,9 & 231 & 42,0 \\
Atípico & 172 & 71,1 & 167 & 100,0 \\
Total & 242 & 100,0 & 398 & \\
& & & & 50,5 \\
Censura & & & & 49,5 \\
0 & 194 & 80,2 & 201 & 100,0 \\
1 & 48 & 19,8 & 197 & \\
Total & 242 & 100 & 398 & \\
& & & &
\end{tabular}

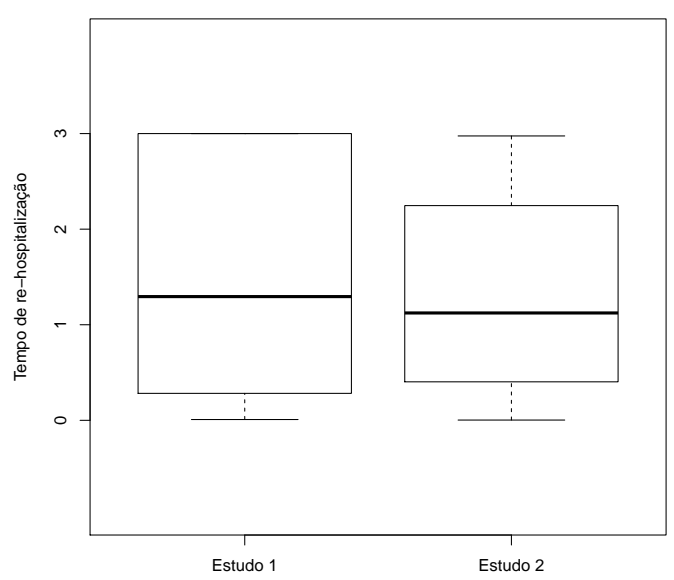

Figura 3.25: Distribuição do tempo de re-hospitalização (anos) em ambos os estudos.

O objetivo dos estudos foi investigar se o tipo de antipsicótico influencia no tempo de re- 
hospitalização (anos) e, caso o tipo influencie, qual o antipsicótico que mantém por mais tempo o paciente fora do Hospital. Para tal, foi ajustado um modelo de regressão paramétrico para dados com censura à direita. A análise de regressão de tempos de vida envolve especificações para a distribuição desse tempo, $T$, dado um vetor de covariáveis $\boldsymbol{z}$. Estes modelos podem ser linearizados tomando logaritmo:

$$
\ln (T)=\alpha_{0}+\alpha_{1} z_{1}+\ldots+\alpha_{p} z_{p}+\epsilon=z^{\prime} \boldsymbol{\alpha}+\epsilon,
$$

onde $z^{\prime}=\left(1, z_{1}, z_{2}, \ldots, z_{p}\right)$ é o vetor de covariáveis, $\boldsymbol{\alpha}=\left(\alpha_{0}, \alpha_{1}, \ldots, \alpha_{p}\right)^{\prime}$ é o vetor de parâmetros do modelo a ser estimado e $\epsilon$ é o erro aleatório. A distribuição do erro pode ser especificada, por exemplo, pelas distribuições Exponencial, Log-normal, Log-logística e Weibull.

A distribuição fundamental proposta para modelos de sobrevivência é a distribuição Weibull, em razão da sua flexibilidade. O modelo de regressão Weibull é um dos mais utilizados na modelagem de sobrevivência para investigar o impacto de outros fatores no tempo de vida e pode ser representado da seguinte maneira:

$$
\ln (T)=\boldsymbol{z}^{\prime} \boldsymbol{\alpha}+\sigma v
$$

onde $\eta=\exp \left(\boldsymbol{z}^{\prime} \boldsymbol{\alpha}\right)$ e o erro $v=\ln (\epsilon)$ tem distribuição do valor extremo com parâmetro de escala $\sigma=\beta^{-1}$ (Lawless, 2003). Portanto, a função de sobrevivência para $Y$ condicional a $\boldsymbol{z}$ é expressa por

$$
S(\ln (t) \mid \boldsymbol{z})=\exp \left\{-\exp \left\{\frac{\ln (t)-\boldsymbol{z}^{\prime} \boldsymbol{\alpha}}{\sigma}\right\}\right\}
$$

e, para $T$ condicional a $\boldsymbol{z}$, por

$$
S(t \mid \boldsymbol{z})=\exp \left\{-\left(\frac{t}{\exp \left\{\boldsymbol{z}^{\prime} \boldsymbol{\alpha}\right\}}\right)^{\beta}\right\}
$$

Como se sabe, na análise de sobrevivência, a variável resposta é sempre o tempo até a ocorrência de um evento, denominado tempo de sobrevivência. Neste exemplo, nossa variável resposta é o tempo até a re-hospitalização do paciente com esquizofrenia e com o mecanismo de censura à direita. Podese escrever a função de verossimilhança dada em (3.5), para o $j$-ésimo estudo $(j=1,2)$, do seguinte modo

$$
L_{j}\left(\boldsymbol{t}_{j}, \alpha\right)=\prod_{i=1}^{n_{j}} f\left(t_{j}, \alpha\right)^{\delta_{i j}}\left[S\left(t_{j}, \alpha\right)\right]^{1-\delta_{i j}} .
$$

Portanto, a função de verossimilhança para os dados de sobrevivência censurados à direita utilizando o modelo de regressão Weibull (3.47), para ambos os estudos, é dada por:

$$
L_{j}\left(\boldsymbol{t}_{j}, \beta, \eta\left(\boldsymbol{z}_{j}\right)\right)=\prod_{i=1}^{n_{j}}\left[\frac{\beta}{\eta\left(\boldsymbol{z}_{j}\right)^{\beta}} t_{j}^{\beta-1} \exp \left[-\left(\frac{t_{j}}{\eta\left(\boldsymbol{z}_{j}\right)}\right)^{\beta}\right]^{\delta_{i j}}\left[\exp \left[-\left(\frac{t_{j}}{\eta\left(\boldsymbol{z}_{j}\right)}\right)^{\beta}\right]^{\delta_{i j}}\right]^{1-\delta_{i j}},\right.
$$

onde $\eta\left(\boldsymbol{z}_{j}\right)=\exp \left\{\alpha_{0}+\alpha_{1} z_{j}\right\}, z_{j}$ é a covariável que representa o tipo de antipsicótico no $j$-ésimo estudo $\left(z_{j}=0\right.$, se antipsicótico típico e $z_{j}=1$, se antipsicótico atípico), $n_{j}$ é o tamanho amostral e $\delta_{i j}$ é uma variável aleatória indicadora de censura para o $j$-ésimo estudo. Neste caso, $\delta_{i j}$, é a variável indicadora da re-hospitzalização. Ou seja, para o estudo $j,(j=1,2), \delta_{i j}$ assume valor 1 para o $i$-ésimo paciente se o mesmo re-hospitalizou e, caso contrário, $\delta_{i j}=0$.

Para se encontrar a distribuição a posteriori conjunta dos parâmetros $(\boldsymbol{\alpha}, \beta)$, primeiramente é preciso definir a função a priori dos mesmos. Para tal, considera-se que os parâmetros regressores seguem, a priori, distribuição Normal com média 0 e desvio-padrão 1.000 e, para $\beta$ considera-se a priori não informativa de Jeffreys, $\pi(\beta) \propto \frac{1}{\beta}$. Logo, a priori conjunta de $(\boldsymbol{\alpha}, \beta)$ é

$$
\pi\left(\beta, \eta\left(\boldsymbol{z}_{j}\right)\right) \propto \pi(\beta) \pi\left(\alpha_{0}\right) \pi\left(\alpha_{1}\right) .
$$


Portanto, a distribuição a posteriori conjunta dos parâmetros em questão, para o $j$-ésimo estudo, é dada por

$$
\pi_{j}\left(\beta, \eta\left(\boldsymbol{z}_{j}\right)\right) \propto L_{j}\left(\boldsymbol{t}_{j} \beta, \eta\left(\boldsymbol{z}_{j}\right)\right) \pi\left(\beta, \eta\left(\boldsymbol{z}_{j}\right)\right) .
$$

Para uma metanálise com dados de sobrevivência considerando o modelo regressão Weibull com dados de censura à direita, a distribuição metanalítica de $\left(\beta, \eta\left(\boldsymbol{z}_{j}\right)\right)$ é dada por:

$$
\pi_{M}(\beta, \eta(\boldsymbol{z}) \mid \boldsymbol{t})=\sum_{j=1}^{J} \omega_{j} \pi_{j}\left(\beta, \eta\left(\boldsymbol{z}_{j}\right) \mid \boldsymbol{t}_{j}\right),
$$

em que $\omega_{j}=\frac{n_{j}}{\sum_{j=1}^{J} n_{j}}$ é o peso do $j$-ésimo estudo.

Para se estimar os parâmetros em questão e as curvas de sobrevivência para cada estudo, assim como as medidas metanalíticas, foi obtida uma amostra das distribuições a posteriori marginais de $\alpha_{0}, \alpha_{1}$ e $\beta$ para ambos os estudos, utilizando o algoritmo de Metropolis-Hastings. As medidas-resumo (média, desvio-padrão (DP) e intervalo de 95\% de credibilidade (Int. Cred. 95\%)) das amostras de todas as cadeias simuladas, adotando aquecimento (burn in) de 10.000, com tamanho inicial de 100.000 e salto igual a 10, encontram-se na Tabela 3.16. Note que o tipo de antipsicótico não interfere no tempo de re-hospitalização. As funções densidade a posteriori marginal estimadas dos parâmetros $\beta, \alpha_{0}$ e $\alpha_{1}$, assim como, a metanalítica, encontram-se na Figura 3.26. Posteriormente, para cada estudo, foram ajustadas as curvas de sobrevivência para cada tipo de antipsicótico via Algoritmo 1 (Figura 3.27(a) e Figura 3.27(b)) e suas respectivas curvas de sobrevivência metanalítica (Figura $3.27(\mathrm{c})$ ), via Algoritmo 2. Por meio da Figura 3.27, observa-se que as curvas de sobrevivência para os tipos de antipsicóticos são muito próximas em ambos os estudos e na metanálise, confirmando que não há influência do tipo de antipsicótico no tempo de re-hospitalização. O mesmo pode ser observado por meio das probabilidades de re-hospitalização. As probabilidades de um paciente esquizofrênico que utiliza antipsicótico típico re-hospitalizar, após 1 ano para o estudo 1, estudo 2 e metanálise são, respectivamente, iguais a $0,85,0,62$ e 0,71. Já, as probabilidades de um paciente esquizofrênico que utiliza antipsicótico atípico re-hospitalizar após um ano para o estudo 1, estudo 2 e metanálise são, respectivamente, iguais a $0,86,0,67$ e 0,73 .

Tabela 3.16: Medidas-resumo a posteriori para os parâmetros de interesse.

\begin{tabular}{cccccccc}
\hline & \multicolumn{3}{c}{ Estudo 1 } & & \multicolumn{3}{c}{ Estudo 2 } \\
\cline { 2 - 3 } \cline { 6 - 8 } & Média & DP & Int. Cred. 95\% & & Média & DP & Int. Cred. 95\% \\
\hline$\beta$ & 0,77 & 0,10 & {$[0,59 ; 0,98]$} & & 0,59 & 0,04 & {$[0,53 ; 0,68]$} \\
$\alpha_{0}$ & 2,48 & 0,48 & {$[1,68 ; 3,53]$} & & 1,23 & 0,16 & {$[0,93 ; 1,57]$} \\
$\alpha_{1}$ & 0,04 & 0,46 & {$[-0,93 ; 0,91]$} & & 0,22 & 0,24 & {$[-0,25 ; 0,71]$} \\
\hline
\end{tabular}


(a)

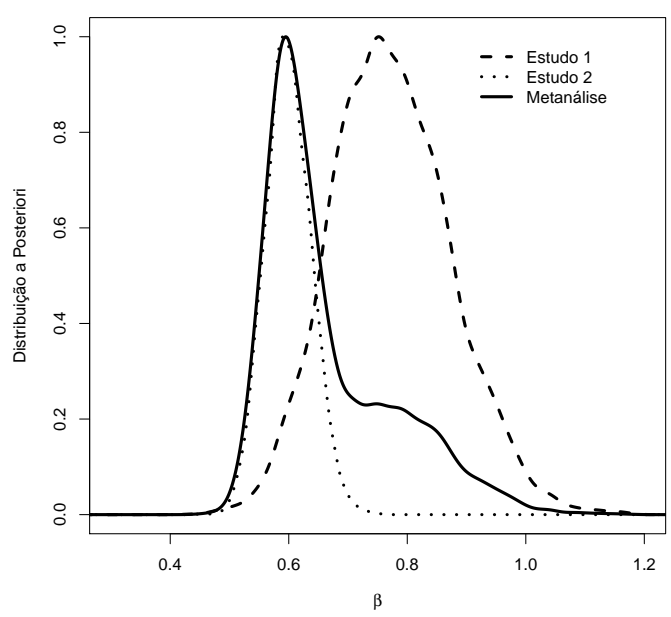

(b)

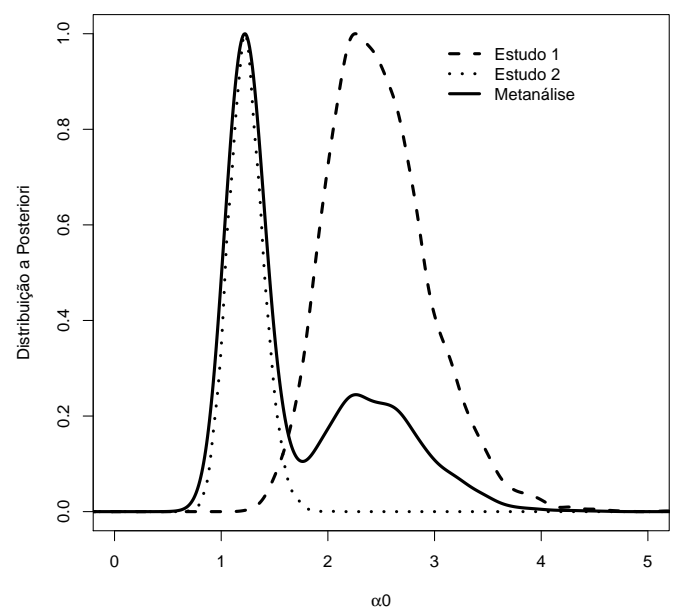

(c)

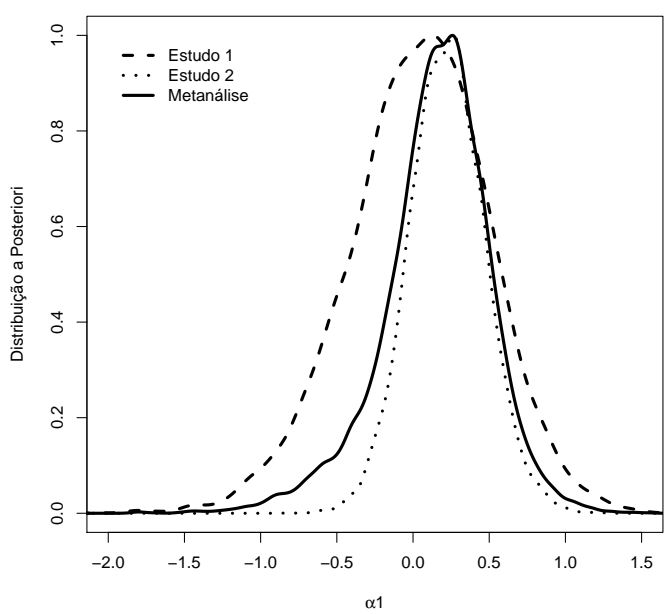

Figura 3.26: (a) Distribuições a posteriori de $\beta$. (b) Distribuições a posteriori de $\alpha_{0}$. (c) Distribuições a posteriori de $\alpha_{1}$. 

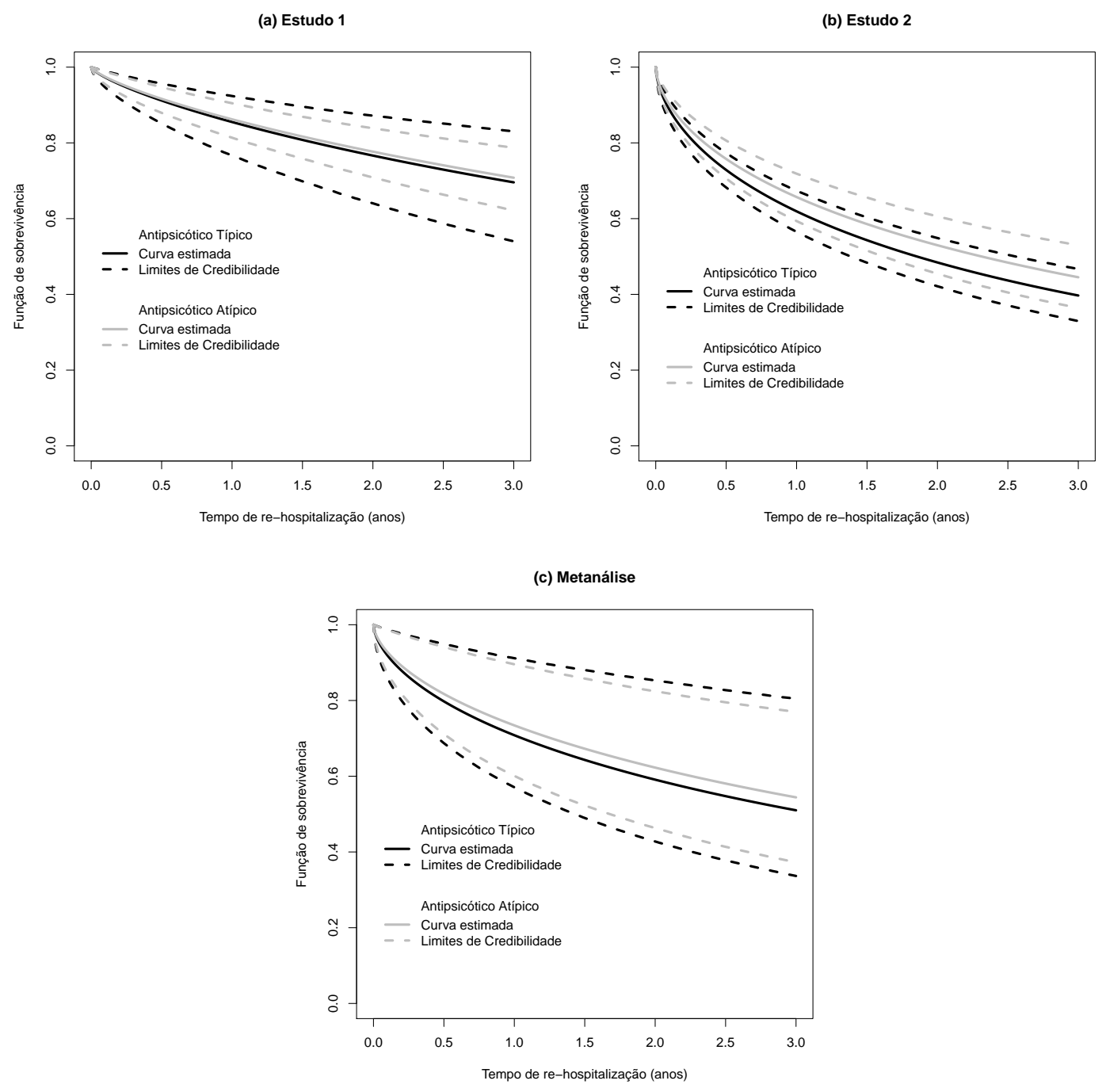

Figura 3.27: Curvas de sobrevivência relacionadas aos Estudos 1 (a), Estudo 2 (b) e metanálise (c).

\subsection{Discussão}

O tratamento inadequado dos dados de sobrevivência na realização de uma metanálise é comum. Então, como uma alternativa aos métodos existentes, utiliza-se a medida metanalítica proposta no Capítulo 2, para a realização de metanálise com dados de sobrevivência completos ou censurados. Percebe-se que, para os modelos Exponencial e Log-normal, realizar uma metanálise baseada na literatura com dados de sobrevivência é equivalente a realizar uma metanálise caso a caso, o que parecia impossível em análises com esses tipos de dados.

Na modelagem do tempo de vida são utilizados alguns modelos estatísticos, entre eles, o modelo Weibull, que é bastante empregado na análise de dados de sobrevivência em razão de sua flexibilidade na acomodação dos diversos tipo de taxas de falha. Geralmente, as curvas de sobrevivência são estimadas por meio de estimadores plug-in (Polpo et al. [2009]), o que impossibilita a construção de uma banda de credibilidade para as mesmas. Portanto, as funções de sobrevivência e suas bandas de credibilidade são estimadas via Metropolis-Hastings. A curva de sobrevivência metanalítica também foi encontrada via Metropolis-Hastings considerando as distribuições a posteriori metanalíticas dos parâmetros do modelo utilizado. 


\section{Capítulo 4}

\section{Considerações finais e pesquisas futuras}

Os resultados desta tese basicamente resumem-se nos seguintes aspectos:

- Uma medida metanalítica bayesiana foi proposta como alternativa aos métodos utilizados. Esta medida é uma mistura de distribuições a posteriori do parâmetro de interesse, $\theta$, de cada estudo. O método proposto produz como medida metanalítica uma distribuição de probabilidade a posteriori de $\theta$. Por meio desta distribuição podem ser calculadas a esperança e a variância a posteriori de $\theta$, além de outras medidas.

- Observou-se que a metodologia proposta é uma medida geral, fazendo com que ela possa ser utilizada, desde o problema mais trivial de metanálise aos mais complexos. Além disso, quando há estatística suficiente disponível, realizar uma metanálise baseada na literatura é equivalente a realizar uma metanálise caso a caso, sem perda de informação. O que resulta em um ganho de qualidade para a metanálise baseada na literatura, uma vez que a metanálise caso a caso é considerada o padrão ouro.

- A metodologia proposta foi utilizada para resolver problemas de metanálise com dados de sobrevivência. Observou-se que, para os modelos Exponencial e Log-normal, realizar metanálise caso a caso com dados de sobrevivência é equivalente a fazer uma metanálise baseada na literatura, o que parecia difícil em análises com esses tipos de dados.

- Na modelagem do tempo de vida, utilizou-se o modelo Weibull. As curvas de sobrevivência e suas bandas de credibilidade foram estimadas via Metropolis-Hastings, assim como a curva de sobrevivência metanalítica.

Vários trabalhos poderão ser desenvolvidos apoiados nas ideias e resultados desta tese. Alguns deles são citados:

- Aperfeiçoamento computacional para a realização da metanálise proposta;

- Aplicações em outros tipos de modelos estatísticos;

Espera-se que, por meio dos resultados desta tese, tenhamos contribuído de forma significativa na pesquisa do aperfeiçoamento de métodos utilizados em metanálise, e que futuros trabalhos sejam frutos das ideias aqui discutidas. 


\section{Apêndice A}

\section{Anexo}

Neste capitulo encontram-se os conjuntos de dados utilizados ao longo da tese.

Tabela A.1: Dados referente ao Exemplo 2 - Capítulo 3

\begin{tabular}{lcccccccccccc}
\hline & 19.6 & 13.5 & 12.7 & 14.2 & 15.3 & 13.1 & 21.8 & 18.0 & 8.4 & 10.6 & 10.0 & 13.5 \\
Estudo 1 & 11.5 & 14.0 & 13.3 & 12.0 & 12.2 & 15.0 & 14.4 & 9.9 & 15.8 & 15.7 & 12.2 & 14.3 \\
& 12.6 & 12.0 & 10.9 & 8.9 & 9.7 & 11.1 & 11.9 & 11.1 & 7.3 & 16.2 & 13.3 & 14.4 \\
& 8.8 & 14.5 & 12.4 & 16.5 & & & & & & & & \\
\hline \multirow{2}{*}{ Estudo 2 } & 19.3 & 22.7 & 13.2 & 21.5 & 23.4 & 25.1 & 19.1 & 19.1 & 23.2 & 15.9 & 22.8 & 15.5 \\
& 20.9 & 20.7 & 23.3 & 32.3 & 22.8 & 18.5 & 19.8 & 22.9 & 18.1 & 16.0 & 18.6 & 18.0 \\
& 22.1 & 14.9 & 20.5 & 24.1 & 18.7 & 26.0 & 19.4 & 23.2 & 19.7 & 18.2 & 16.8 & 19.7 \\
& 18.8 & 15.8 & 19.7 & 18.4 & & & & & & & & \\
\hline \multirow{2}{*}{ Estudo 3 3} & 25.9 & 14.4 & 11.3 & 23.1 & 18.8 & 13.3 & 20.0 & 15.5 & 12.2 & 17.9 & 16.4 & 28.3 \\
& 17.3 & 19.1 & 13.9 & 13.9 & 14.8 & 22.1 & 9.1 & 10.0 & 21.3 & 12.8 & 14.1 & 11.8 \\
& 18.8 & 14.5 & 14.5 & 17.2 & 17.2 & 13.1 & 20.6 & 14.7 & 16.5 & 15.1 & 16.7 & 17.1 \\
& 19.8 & 15.2 & 13.2 & 13.4 & & & & & & & & \\
\hline
\end{tabular}

Tabela A.2: Dados referente ao Exemplo 3 - Capítulo 3

\begin{tabular}{llllllllllll}
\hline & 0,75 & 1,01 & 0,33 & 0,60 & 0,49 & 1,17 & 0,49 & 0,32 & 1,31 & 1,40 & 0,40 \\
Estudo 1 & 0,19 & 0,26 & 1,78 & 0,70 & 0,88 & 0,61 & 0,42 & 0,73 & 1,06 & 0,53 & 0,49 \\
& 1,09 & 1,28 & 1,26 & 1,66 & 0,15 & 1,57 & 0,35 & 0,88 & 0,96 & 0,39 & 0,56 \\
& 1,97 & 0,54 & 1,08 & 2,06 & 0,74 & 0,21 & 0,29 & 0,42 & 0,88 & 1,08 & 0,53 \\
& 0,50 & 2,15 & 1,01 & 1,67 & 1,17 & 0,42 & & & & & \\
& 1,72 & 0,06 & 3,82 & 0,84 & 0,92 & 4,27 & 3,28 & 0,90 & 1,86 & 0,48 & 0,08 \\
Estudo 2 2 & 1,55 & 0,52 & 0,03 & 0,90 & 0,34 & 0,90 & 0,68 & 0,40 & 0,52 & 0,05 & 0,43 \\
& 1,97 & 0,87 & 0,61 & 1,09 & 2,18 & 0,39 & 0,97 & 0,39 & 0,18 & 0,01 & 1,02 \\
& 2,36 & 0,15 & 0,85 & 1,03 & 0,99 & 0,67 & 0,31 & 2,46 & 0,46 & 1,94 & 0,21 \\
& 1,34 & 0,33 & 0,04 & 2,10 & 0,54 & 1,15 & & & & & \\
\hline
\end{tabular}


Tabela A.3: Dados referente ao Exemplo 1 - Capítulo 3.

\begin{tabular}{cccc|cccc}
\hline$T_{1}$ & $\delta_{1}$ & $T_{2}$ & $\delta_{2}$ & $T_{1}$ & $\delta_{1}$ & $T_{2}$ & $\delta_{2}$ \\
\hline 11.31 & 1 & 3.72 & 1 & 1.47 & 0 & 0.66 & 1 \\
1.18 & 1 & 9.57 & 1 & 1.51 & 1 & 0.34 & 0 \\
6.47 & 1 & 6.97 & 1 & 0.27 & 1 & 0.32 & 0 \\
7.87 & 1 & 2.19 & 1 & 3.34 & 1 & 6.59 & 1 \\
1.83 & 1 & 3.43 & 1 & 11.70 & 1 & 2.30 & 1 \\
8.71 & 1 & 1.46 & 1 & 1.01 & 1 & 8.12 & 1 \\
9.51 & 1 & 1.00 & 1 & 1.92 & 1 & 2.69 & 1 \\
3.03 & 0 & 9.60 & 1 & 0.46 & 1 & 1.36 & 1 \\
2.65 & 1 & 2.42 & 1 & 0.09 & 0 & 12.26 & 1 \\
3.19 & 1 & 1.46 & 1 & 3.81 & 1 & 1.49 & 1 \\
7.93 & 1 & 0.36 & 1 & 2.01 & 1 & 2.64 & 1 \\
1.78 & 1 & 4.51 & 1 & 4.34 & 1 & 2.12 & 1 \\
1.16 & 1 & 0.34 & 1 & 5.51 & 1 & 2.57 & 0 \\
5.27 & 1 & 5.21 & 1 & 1.45 & 1 & 1.73 & 1 \\
4.21 & 1 & 3.14 & 1 & 5.34 & 1 & 0.36 & 1 \\
0.02 & 1 & 4.87 & 1 & 13.63 & 1 & 0.39 & 1 \\
0.60 & 1 & 5.13 & 1 & 6.50 & 1 & 0.81 & 0 \\
4.02 & 1 & 14.18 & 1 & 3.67 & 1 & 3.66 & 0 \\
4.52 & 0 & 0.00 & 0 & 9.80 & 1 & 10.96 & 1 \\
2.13 & 0 & 3.89 & 1 & 4.46 & 1 & 0.09 & 1 \\
0.51 & 1 & 4.03 & 1 & 1.34 & 1 & 0.06 & 0 \\
9.37 & 1 & 4.24 & 1 & 9.14 & 1 & 4.72 & 1 \\
6.72 & 1 & 4.67 & 1 & 7.04 & 1 & 7.41 & 1 \\
2.31 & 1 & 0.30 & 1 & 1.90 & 1 & 1.65 & 1 \\
0.46 & 0 & 1.22 & 1 & 4.37 & 1 & 0.21 & 1 \\
4.81 & 1 & 1.14 & 1 & 2.61 & 1 & 5.51 & 1 \\
1.61 & 1 & 0.36 & 1 & 3.04 & 1 & 6.01 & 1 \\
16.25 & 1 & 5.69 & 0 & 2.74 & 1 & 1.43 & 1 \\
2.30 & 1 & 4.09 & 1 & 1.07 & 1 & 1.50 & 1 \\
3.76 & 1 & 0.16 & 1 & 3.06 & 1 & 2.46 & 1 \\
0.99 & 1 & 0.69 & 1 & 12.83 & 1 & 6.02 & 1 \\
3.97 & 1 & 0.16 & 1 & 4.14 & 1 & 2.16 & 0 \\
0.56 & 1 & 2.75 & 0 & 2.21 & 1 & 5.52 & 1 \\
1.34 & 1 & 3.89 & 1 & 9.50 & 1 & 1.71 & 0 \\
3.42 & 1 & 0.13 & 0 & 16.94 & 1 & 12.54 & 1 \\
0.10 & 1 & 0.42 & 0 & 0.84 & 1 & 1.16 & 1 \\
0.29 & 1 & 5.52 & 1 & 3.16 & 1 & 1.10 & 1 \\
4.31 & 0 & 0.32 & 1 & 6.74 & 1 & 4.07 & 1 \\
4.56 & 1 & 5.87 & 1 & 4.91 & 1 & 4.83 & 1 \\
18.20 & 1 & 1.83 & 1 & 5.88 & 0 & 0.17 & 1 \\
2.58 & 0 & 4.81 & 1 & 4.04 & 1 & 0.88 & 0 \\
0.79 & 1 & 3.54 & 1 & 3.75 & 0 & 22.45 & 1 \\
4.26 & 1 & 2.34 & 1 & 0.05 & 1 & 21.74 & 1 \\
5.34 & 1 & 6.46 & 0 & 1.56 & 1 & 0.84 & 1 \\
2.85 & 0 & 4.97 & 1 & 2.21 & 1 & 0.23 & 1 \\
8.18 & 1 & 9.21 & 1 & 0.67 & 1 & 7.65 & 1 \\
4.93 & 1 & 12.34 & 1 & 0.45 & 1 & 5.69 & 1 \\
11.56 & 1 & 2.29 & 1 & 2.83 & 1 & 2.22 & 1 \\
2.56 & 1 & 3.84 & 1 & 1.99 & 1 & 8.26 & 0 \\
2.00 & 1 & 2.47 & 1 & 3.51 & 1 & 1.89 & 1 \\
\hline & & & & & & &
\end{tabular}


Tabela A.4: Dados referente ao Exemplo 4 - Capitulo 3

\begin{tabular}{|c|c|c|c|c|c|c|c|c|c|c|c|}
\hline \multicolumn{4}{|c|}{ Estudo 1} & \multicolumn{4}{|c|}{ Estudo 2} & \multicolumn{4}{|c|}{ Estudo 3} \\
\hline Tempo & Fator & Tempo & Fator & Tempo & Fator & Tempo & Fator & Tempo & Fator & Tempo & Fator \\
\hline 0,88 & 1 & 4,67 & 2 & 2,98 & 2 & 6,27 & 3 & 8,02 & 1 & 9,32 & 1 \\
\hline 7,07 & 1 & 8,87 & 2 & 7,64 & 3 & 4,33 & 2 & 8,16 & 3 & 7,73 & 2 \\
\hline 3,64 & 1 & 6,1 & 2 & 6,21 & 1 & 5,51 & 1 & 5,84 & 3 & 7,75 & 1 \\
\hline 6,79 & 1 & 7,87 & 2 & 6,01 & 1 & 9,88 & 2 & 9,37 & 3 & 6,29 & 3 \\
\hline 6,36 & 1 & 5,57 & 2 & 4,57 & 1 & 5,52 & 1 & 7,52 & 2 & 5,11 & 2 \\
\hline 5,3 & 1 & 8,67 & 2 & 5,98 & 2 & 4,54 & 1 & 7,55 & 3 & 4,77 & 1 \\
\hline 5,77 & 1 & 2,91 & 2 & 4,67 & 1 & 4,52 & 1 & 10,06 & 3 & 6,31 & 2 \\
\hline 5,87 & 1 & 1,64 & 2 & 5,41 & 1 & 8,06 & 2 & 9,17 & 1 & 7,32 & 1 \\
\hline 2,71 & 1 & 7,46 & 2 & 10,49 & 1 & 12,05 & 3 & 9,59 & 2 & 5,68 & 1 \\
\hline 5,97 & 1 & 4,07 & 2 & 7,17 & 1 & 8,13 & 1 & 6,37 & 1 & 9,8 & 3 \\
\hline 3,3 & 1 & 8,28 & 2 & 8,36 & 3 & 8,77 & 3 & 0,93 & 2 & 8,38 & 1 \\
\hline 2,21 & 1 & 2,58 & 2 & 11,41 & 2 & 4,65 & 2 & 3,68 & 3 & 7,11 & 2 \\
\hline 3,59 & 1 & 7,08 & 2 & 5,02 & 1 & 5,1 & 1 & 8,37 & 3 & 3,53 & 2 \\
\hline 6,83 & 1 & 6,33 & 2 & 5,56 & 3 & 8,5 & 3 & 2,4 & 2 & 6,22 & 1 \\
\hline 7,35 & 1 & 6,8 & 2 & 6,63 & 2 & 4,98 & 2 & 5,8 & 2 & 6,38 & 3 \\
\hline 4,52 & 1 & 11,15 & 2 & 3,64 & 1 & 6,5 & 1 & 4,56 & 1 & 9,35 & 1 \\
\hline 0,95 & 1 & 9,51 & 3 & 6,16 & 1 & 4,79 & 2 & 4,15 & 1 & 9,93 & 3 \\
\hline 7,03 & 1 & 10,54 & 3 & 5,96 & 3 & 7,12 & 3 & 6,02 & 3 & 5,57 & 1 \\
\hline 6,48 & 1 & 6,44 & 3 & 5,95 & 3 & 4 & 1 & 2,19 & 1 & 6 & 3 \\
\hline 7,52 & 1 & 10,47 & 3 & 4,28 & 2 & 8,28 & 2 & 6,86 & 1 & 6,99 & 2 \\
\hline 3,59 & 1 & 4,1 & 3 & 6,38 & 3 & 9,46 & 3 & 7,27 & 3 & 5,2 & 2 \\
\hline 2,72 & 1 & 6,51 & 3 & 5,81 & 1 & 9,22 & 1 & 9,35 & 2 & 4,6 & 1 \\
\hline 4,2 & 1 & 7 & 3 & 9,96 & 3 & 7,86 & 1 & 5,73 & 2 & 6,62 & 2 \\
\hline 4,33 & 1 & 9,25 & 3 & 4,9 & 2 & 9,89 & 3 & 7,52 & 3 & 5,6 & 2 \\
\hline 5,12 & 1 & 6 & 3 & 4,75 & 1 & 6,89 & 2 & 3,79 & 1 & 9,73 & 2 \\
\hline 7,16 & 1 & 3,74 & 3 & 2,26 & 1 & 6,38 & 2 & 7,43 & 3 & 3,61 & 1 \\
\hline 2,5 & 1 & 8,13 & 3 & 5,52 & 1 & 6,99 & 1 & 7,88 & 1 & 8,78 & 3 \\
\hline 5,3 & 1 & 4,64 & 3 & 4,25 & 1 & 5,52 & 1 & 8,66 & 2 & 6,21 & 2 \\
\hline 6,73 & 1 & 4,46 & 3 & 7,34 & 2 & 10,49 & 2 & 2,12 & 2 & 6,94 & 1 \\
\hline 4,64 & 1 & 4,12 & 3 & 6,57 & 2 & 5,01 & 2 & 7,05 & 1 & 8,14 & 3 \\
\hline 2,56 & 1 & 8,87 & 3 & 8,06 & 3 & 4,73 & 1 & 7,19 & 1 & & \\
\hline 1,49 & 1 & 7,33 & 3 & 6,6 & 1 & 5,67 & 1 & 6,84 & 2 & & \\
\hline 3,89 & 1 & 5,28 & 3 & 4,71 & 3 & 5,96 & 2 & 9,87 & 2 & & \\
\hline 9,58 & 1 & 8,13 & 3 & 3,82 & 2 & 7,96 & 3 & 8,63 & 2 & & \\
\hline 6,85 & 1 & 5,68 & 3 & 5,36 & 1 & 9,14 & 3 & 8,46 & 3 & & \\
\hline 6,01 & 1 & 5,01 & 3 & 5,28 & 1 & 3,96 & 2 & 6,29 & 3 & & \\
\hline 5,44 & 1 & 4,42 & 3 & 9,68 & 3 & 11 & 3 & 6,28 & 1 & & \\
\hline 7,56 & 1 & 8,32 & 3 & 4,15 & 2 & 1,81 & 1 & 6,11 & 1 & & \\
\hline 3,36 & 1 & 8,48 & 3 & 6,95 & 1 & 6,86 & 1 & 7,36 & 2 & & \\
\hline 4,1 & 1 & 5,82 & 3 & 7,88 & 2 & 10,68 & 2 & 8,01 & 3 & & \\
\hline 3,98 & 1 & & & 6,11 & 1 & 9,9 & 3 & 5,64 & 1 & & \\
\hline 3,46 & 1 & & & 8,07 & 2 & 6,48 & 2 & 6,08 & 3 & & \\
\hline 2,13 & 1 & & & 3,44 & 1 & 8,14 & 2 & 9,42 & 2 & & \\
\hline 2,96 & 1 & & & 7,52 & 2 & 9,1 & 3 & 3,85 & 2 & & \\
\hline 5,26 & 2 & & & 6,85 & 3 & 7,65 & 2 & 9,31 & 3 & & \\
\hline 5,11 & 2 & & & 8,85 & 2 & 7,97 & 1 & 5,3 & 3 & & \\
\hline 7,39 & 2 & & & 6,57 & 2 & 4,88 & 2 & 2,63 & 2 & & \\
\hline 4,47 & 2 & & & 5,29 & 1 & 4,42 & 2 & 3,03 & 1 & & \\
\hline 3,88 & 2 & & & 6,87 & 3 & 9,38 & 1 & 8,96 & 3 & & \\
\hline 9,9 & 2 & & & 7,71 & 2 & 6,66 & 1 & 3,36 & 1 & & \\
\hline 3,66 & 2 & & & 8,68 & 1 & 7,25 & 3 & 4,56 & 1 & & \\
\hline 8,04 & 2 & & & 9,75 & 3 & 8,15 & 3 & 10,37 & 3 & & \\
\hline 2,77 & 2 & & & 8,07 & 3 & 3,73 & 1 & 6,83 & 2 & & \\
\hline 10,08 & 2 & & & 5,67 & 3 & 6,37 & 1 & 3,87 & 2 & & \\
\hline 5,31 & 2 & & & 6,09 & 1 & 8,29 & 2 & 8,34 & 3 & & \\
\hline 4,16 & 2 & & & 6,96 & 3 & 9,33 & 3 & 4,24 & 1 & & \\
\hline 8,59 & 2 & & & 6,93 & 3 & 7,33 & 3 & 5,79 & 2 & & \\
\hline 5,01 & 2 & & & 6,78 & 1 & 6,38 & 2 & 8,04 & 2 & & \\
\hline 6,26 & 2 & & & 7,09 & 2 & 7,23 & 2 & 6,97 & 1 & & \\
\hline 7,34 & 2 & & & 6,7 & 3 & 10,48 & 1 & 5,55 & 1 & & \\
\hline
\end{tabular}


APÊNDICE A 


\section{Referências Bibliográficas}

Alderson, P; Green, S (2002). Cochrane Collaboration open learning material for reviewers. The Cochrane Collaboration. 2

Atallah, AN (1998). Revisão sistemática da literatura e metanálise. In: Atallah, A N; Castro, A A (ed.). Medicina baseada em evidências: fundamentos da pesquisa clínica. Lemos-Editorial, 42-48. 2

Berry, DA (1989). A Bayesian Approach to Multicenter Trials and Meta-analysis. National Science Foundation, Washington, D.C. 12, 14

Campbell, S; Marriott, M; Nahmias, C; McQueen, GM (2004). Lower hippocampal volume in patients suffering from depression: a meta-analysis. Am J Psychiatry, 161, 598-607. 1

Cochran, WG (1937). Problems arising in the analysis of a series of similar experiments. Journal of Royal Statistical Society Supplement 4(1), 102-118. 1

Colosimo, EA; Giolo, SR (2006). Análise de sobrevivência aplicada. ABE - Projeto Fisher, Editora Edgard Blucher. 28, 29, 31, 36

Cox, DR; Snell, EJ (1981). Applied Statistics, Science Paperbacks. Chapman and Hall, London. 31

DerSimonian, R and Laird, N (1986). Meta-Analysis in Clinical Trials. Controlled Clinical Trials, 7, 177-188. 1, 2, 12, 13

Dutton, M (2011). Individual patient-level data meta-analysis: A comparison of methods for the diverse populations collaboration data set. PHD thesis. 2, 3

Egger, M; Smith, GD (1997). Meta-analysis: potencials and promise. Britsh Medical Journal, 315, 7119, 1371-1374. 2, 12

Egger, M; Smith, GD; Altman, DG (2009). Systematic Reviews in health care. Meta-analysis in context. BMJ books. 27

Fagard, RH; Staessen, JA; Thijs, L (1996). Advantages and disadvantages of the meta-analysis approach. Journal of hypertension, 14. 5

Fisher, RA (1932). Statistical Methods for Research Workers. London, Oliver and Boyd, 4th ed. 1

Gan, WQ; Man, SFP; Senthilselvan, A and Sin, DD (2004). Association between chronic obstructive pulmonary disease and systemic inflammation: a systematic review and a metaanalysis. Thorax, $\mathbf{5 9}, 574-580$.

Glass, GV (1976). Primary, secondary, and meta-analysis of research. Educ Res, 5, 3-8. 1, 5

Glass, GV; McGaw B; Smith, ML (1981). Meta-analysis in social research. Beverly Hills: Sage Publications. 1

Hedges, LV (1982). Estimation of effect size from a series of independent experiments. Psychol Bull, 92, 490-499. 1 
Hedges, LV (1983). Combining independent estimators in research synthesis. Br J Math Stat Psychol, 36, 123-131. 1

Herceg, M; Jukic, V; Vidovic, D; Erdeljic, V; Celic, I; Kozumplik,O; Bagaric, D; Radic, MS (2008). Two-year Rehospitalization Rates of Patients with Newly Diagnosed or Chronic Schizophrenia on Atypical or Typical Antipsychotic Drugs: Retrospective Cohort Study. Croatian Medical Journal 49, 215-223. 55

Higgins, JPT and Greens, S (2008). Cochrane Handbook for Systematic Reviews of Interventions: Cochrane Book Series. Chichester: John Wiley \& Sons. 3, 5

Hunter, JE; Schmidt, FL; Jackson, BG (1982). Meta-analysis : Cumulating research findings across studies. Beverly Hills, Calif, Sage Publications. 1

Ibrahim, JG; Chen M-H; Sinha, D (2005). Bayesian Survival Analysis. Springer Series in Statistics, Second Ed. 6

Justo, LP; Soares, BGO e Calil, HM (2005). Revisão sistemática, metanálise e medicina baseada em evidências: considerações conceituais. Jornal Brasileiro de Psquiatria, 53 (3), 242-247. 2, 12

Klein, JP; Moeschberger,ML (1997). Survival Analysis: Techniques for Censored and Truncated Data. Springer-Verlag, New York. 29

Kleinbaum, DG; Klein, M (2005). Survival Analysis - A Self-Learning Text. Ed. Springer, Second Edition. 29

Lau, J; Ioannidis, JPA; Schmid, CH (1997). Quantitative synthesis in systematic reviews. Annals of Internal Medicine, 127 (9), 820-826. 1

Lawless, JF (2003). Statistical models and methods for lifetime data. Wiley Series in Probability and Statistics, Second Edition, John Wiley Sons, Inc. 29

Leandro, G (2005). Meta-analysis in Medical Research. The handbook for the understanding and ractice of meta-analysis BMJ Books, Blackwell Publishing. 1,5

Lee, PM (2012). Bayesian Statistics: An Introduction. Fourth edition. Chichester: John Wiley \& Sons. 9,19

Lewis, S; Clarke, M (2001). Forest plots: trying to see the wood and the trees. BMJ 322, 1479-1480. 8

Lindqvist, H (2006). Competing risks. Tech. rep., Norwegian University of Science and Tecnology. 47

Light, RJ (1983). Evaluation Studies. Review Annual, Beverly Hills, Calif, Sage Publications. 8. 1

Light, RJ; Pillemar, DB (1984). Summing Up: The Science of Reviewing Research. Cambridge, Mass, Havard University Press. 1

Lustosa, SAS; Saconato, H; Atallah, AN; Lopes-Filho, GJ and Matos, D (2008). Impact of extended lymphadenectomy on morbidity, mortality, recurrence and 5-year survival after gastrectomy for cancer. Meta-analysis of randomized clinical trials. Acta Cirúrgica Brasileira, 23 (6), 520-530.

Mantel, N and Haenszel, W (1959). Statistical aspects of analysis of data from retrospective studies of disease. Jornal of the National Cancer Institute, 22 (4), 719-748. 2

Martinez, EZ (2007). Metanálise de ensaios clínicos controlados aleatorizados: aspectos quantitativos. Revista Medicina (Ribeirão Preto), 40 (2), 223-235. 1 
Mazin, SC; Martinez, EZ (2009). Modelos estatísticos em Metanálises. Universidade Federal de São Carlos e Embrapa Pecuária Sudeste, 54 $4^{a}$ RBRAS e $13^{\circ}$ SEAGRO. 10

McLachlan, G; Peel, D (2000). Finite Mixture Models. Wiley Series in Probability and Statistics. 15

O'Hagan, A (1991). Baysian Inference. Kendall's advanced theory of statistics, 2B. 11

Pham, JT; Schmitt, BP; Leehey, DJ (2012). Effects of Dual Blockade of the Renin-Angiotensin System in Diabetic Kidney Disease: A Systematic Review and Meta-Analysis. Journal Nephrology Therapeutics, 1-8. 21, 24

Paulino, CD; Turkman, AA; Murteira, B (2003). Estatística Bayesiana. Fundação Calouste Gulbenkian, Lisboa. 7

Pearson, ES (1938). The probability integral transformation for testing goodness of fit and combing independent tests of significance. Biometrika, 30, 134-148. 1

Pereira, CAB; Stern, JM (1999). Evidence and Credibility: Full Bayesian Significance Test for Precise Hypotheses. Entropia, Suiça, 1, 99-110. 21

Pissini, CF (2006). Aplicações em meta-análise sob um enforque bayesiano usando dados médicos. Dissertação de mestrado. 12

Petitti, DB (1994). Meta-analysis, decision analysis, and cost-effectiveness analysis. New York: Oxford University Press. 1

Petitti, DB (2000). Meta-analysis, decision analysis, and cost-effectiveness analysis. Methods for quantitative synthesis in medicine. Second Edition. New York: Oxford University Press. 26

Polpo, A; Coque-JR, M; Pereira, C A B (2009). Statistical analysis for Weibull distributions in presence of right and left censoring. In: 8th International Conference on Reliability, Maintainability and Safety (ICRMS), Chengdu. The Proceedings of 2009 8th International Conference on Reliability, Maintainability and Safety, 1, 219-223. 30, 60

Rodrigues, J; Cancho, VG; De Castro, M (2008). Teoria unificada de análise de sobrevivência. 1. ed. São Paulo-SP: Associação Brasileira de Estatística. 47

Rosenthal, R (1978). Combining results of independent studies. Psychol Bull, 85, 185-193. 1

Schlattmann, P (2009). Medical Applications of finite mixture models. Statistics for Biology and Health, Springer. 12

Schwarz, G (1978). Estimating the Dimension of a Model. Annals of Statistics, 6, 461-464. 15

Stewart LA; Tierney J (2002). To IPD or Not to IPD? Advantages and Disadvantages of Systematic Reviews Using Individual Patient Data. Evaluation And the Health Professions, 25 (1), 76-97. 3

Stewart LA; Parmar, MK (1993). Meta-analysis of literature or of individual patient data: is there a difference? The Lancet 341, 418-422. 3

Tippett, LHC (1931). The Methods of Statistics. London, Williams and Norgate. 1

Weibull, WA (1939). A statistical theory of strength of materials. Ingeniors Vetenskaps Akademien, 151 (3), 4555. 42

Weibull, WA (1951). A statistical distribution of wide applicability. Journal of Applied Mechaniscs, 18, 239297. 42 
Werneck, AP; Hallak, JC; Nakano, E; Elkis, H (2011). Time to rehospitalization in patients with schizophrenia discharged on first generation antipsychotics, non-clozapine second generation antipsychotics, or clozapine. Psychiatry Research (Print) 188, 315-319. 55 